\title{
WestVirginiaUniversity
}

THE RESEARCH REPOSITORY @ WVU

Graduate Theses, Dissertations, and Problem Reports

2013

\section{Skeletal muscle regeneration and the role of Sirtuin 1}

\author{
Brian T. Bennett \\ West Virginia University
}

Follow this and additional works at: https://researchrepository.wvu.edu/etd

\section{Recommended Citation}

Bennett, Brian T., "Skeletal muscle regeneration and the role of Sirtuin 1" (2013). Graduate Theses, Dissertations, and Problem Reports. 4952.

https://researchrepository.wvu.edu/etd/4952

This Thesis is protected by copyright and/or related rights. It has been brought to you by the The Research Repository @WVU with permission from the rights-holder(s). You are free to use this Thesis in any way that is permitted by the copyright and related rights legislation that applies to your use. For other uses you must obtain permission from the rights-holder(s) directly, unless additional rights are indicated by a Creative Commons license in the record and/ or on the work itself. This Thesis has been accepted for inclusion in WVU Graduate Theses, Dissertations, and Problem Reports collection by an authorized administrator of The Research Repository @ WVU. For more information, please contact researchrepository@mail.wvu.edu. 


\title{
Skeletal muscle regeneration and the role of Sirtuin 1
}

\author{
Brian T. Bennett
}

\author{
Thesis submitted to the \\ School of Medicine \\ at West Virginia University \\ in partial fulfillment of the requirements \\ for the degree of
}

\author{
Master of Science \\ in \\ Exercise Physiology
}
Stephen E. Alway, Ph.D., Chair
Randall W. Bryner, EdD
Paul D. Chantler, PhD

Department of Exercise Physiology

Morgantown, WV

2013

Keywords: Skeletal muscle; regeneration; Sirt1; resveratrol Copyright 2013 Brian T. Bennett 


\title{
Abstract \\ Skeletal muscle regeneration and the role of Sirtuin 1
}

\author{
Brian T. Bennett
}

The main objective of this master's thesis project was to elucidate the role of the deacetylase Sirtuin 1 (Sirt1) in skeletal muscle regeneration. We developed two specific aims to accomplish this task, which involved separate studies in order to study the effects of Sirt1 in multiple models of regeneration. The results from these studies may potentially lead to the development of novel therapies to treat various clinical conditions which require muscle regeneration.

Specific aim 1 utilized resveratrol, a Sirt1 activator, as a supplement to improve muscle regeneration following a period of disuse in aged animals. Aged Fisher $344 \times$ Brown Norway rats were hindlimb suspended for a total of fourteen days (HS), whereupon one-half of the animals were sacrificed and the remainder were unloaded and allowed a fourteen day recovery period of normal ambulation $(R)$, at which point they were sacrificed. During the experimental period, each rat received either $1 \mathrm{ml}$ of resveratrol $(125 \mathrm{mg} / \mathrm{kg}$ ) or $1 \mathrm{ml}$ of carboxymethylcellulose dissolved in distilled water (vehicle) via oral gavage at approximately the same time each morning. Additionally, a group of vehicle treated animals maintained normal ambulation throughout the protocol and served as cage controls for the suspended animals.

Resveratrol was not able to attenuate losses in either body or muscle wet weights following HS and did not provide any additional benefits to these measures during the R period. Surprisingly, resveratrol treated animals did not fare as well on a fatigue protocol and few differences were observed upon examination of a force-frequency curve. However, fiber type analyses revealed a shift towards increased type IIA myosin heavy chain (MyHC) fibers following $\mathrm{HS}$. Resveratrol also attenuated decreases in fiber area in type IIA MyHC fibers following HS and in both type IIA and IIB MyHC fibers following the $\mathrm{R}$ period.

Our results from this study indicate a potential for the use of resveratrol in benefitting skeletal muscle regeneration in aged populations following periods of disuse such as extended hospitalizations. Although resveratrol was unable to maintain muscle function as determined by the force characteristics, it clearly benefitted muscle fiber size which is important in this population as the elderly have diminished regenerative abilities. Future research should be aimed at determining the optimal dosage to fully maximize the benefits of this supplement in skeletal muscle regeneration.

Specific aim 2 attempted to establish the role of Sirt1 in muscle regeneration using transgenic mice which either overexpressed $\left(\mathrm{Sirt}_{1}^{+/+}\right.$) or had diminished Sirt1 expression (Sirt $1^{--}$) in the skeletal muscles. Tibialis anterior (TA) muscles were examined either 3 or 7 days following a cardiotoxin (CTX) injection in the animals left TA muscles. The right TA muscles were injected with sterile PBS and were used as intranimal controls for the CTX administration.

Increasing Sirt1 expression did not have an effect on muscle mass or fiber area at either 3 or 7 days. However, increasing its expression attenuated decreases in maximal tetanic force production at both 3 and 7 days post-injury while there were no differences observed in these forces in the Sirt ${ }^{-1-}$ animals. There were no differences observed between any of the groups in BrdU incorporation and furthermore, in apoptosis as determined via TUNEL staining.

Results from this study indicate that Sirt1 may not play a significant role in muscle mass or fiber area during the early stages of muscle regeneration. However, increasing Sirt1 expression was 
beneficial in that it improved muscle force characteristics which would suggest improved functional recovery in these animals. Therefore, although inconclusive, increasing Sirt1 expression may be beneficial at improving skeletal muscle regeneration in that it may reduce functional impairments in muscle viability. Future research may thus be directed at developing therapies to exploit these benefits and be applied toward clinical populations which require skeletal muscle regeneration. 


\section{Table of Contents}

Chapter 1 Introduction 1

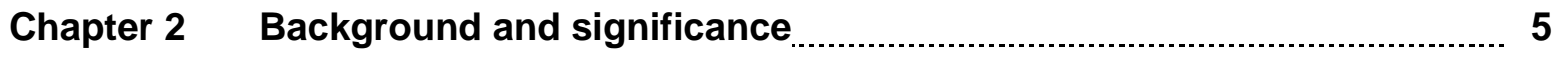

2.1 Skeletal muscle

2.2 Sarcopenia

2.3 Mitochondrial Function and apoptosis

2.4 Satellite cells $\ldots \ldots \ldots \ldots \ldots$

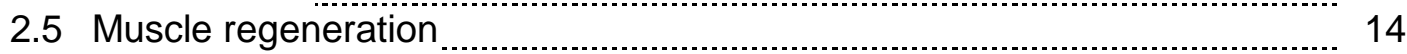

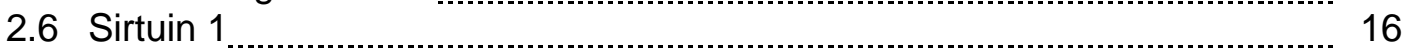

Chapter 3 Effects of resveratrol on disuse associated loss and hindlimb

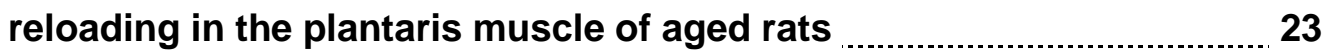

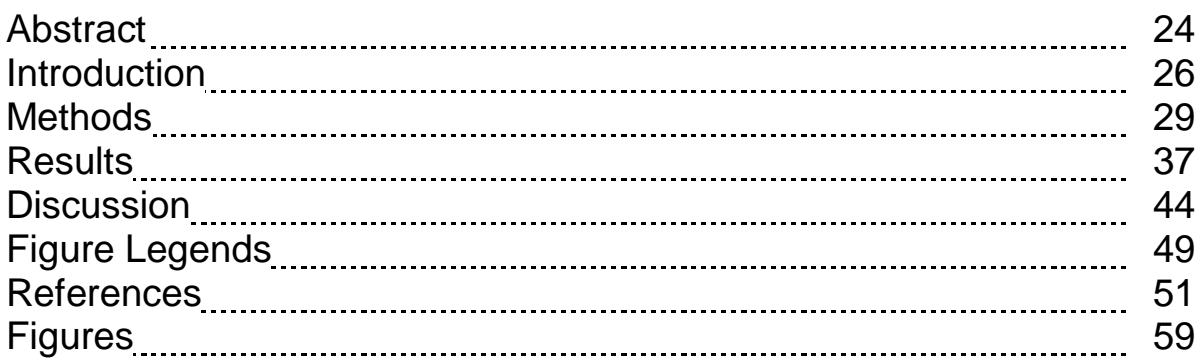

Chapter $4 \quad$ SIRT1 in the regulation of skeletal muscle regeneration following acute myotoxic injury

Abstract 70

Introduction

Methods

Results $\quad 79$

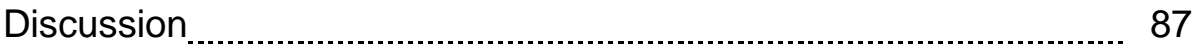

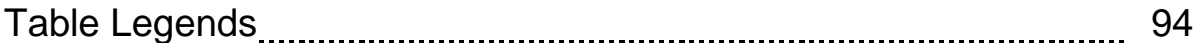

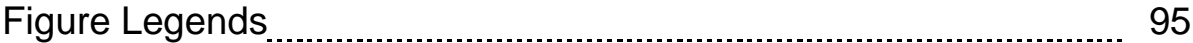

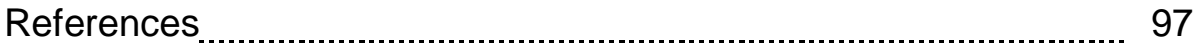

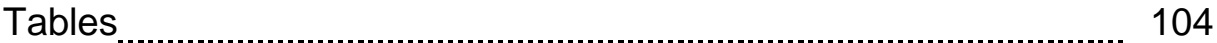

Figures

Chapter 5 Discussion 114

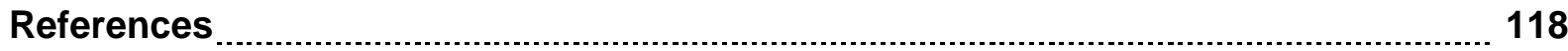




\section{Chapter 1}

\section{Introduction}

Sarcopenia is the involuntary loss of muscle mass and physical function as a consequence of aging (36). Throughout the decades, humans gradually lose increasing amounts of muscle mass, and concomitantly strength. From the age of near 50 and onwards, muscle strength declines approximately $12 \%-15 \%$ each decade (36). Sarcopenia increases the susceptibility to falls and is also associated with increased risk of morbidity and mortality (46). This can severely limit functional independence and therefore, quality of life. Extended hospitalization and other forms of disuse may further exacerbate these complications. Annually, billions of dollars are spent on healthcare costs that can be directly attributed to this condition. While multifactorial in nature, the etiology of sarcopenia may be due in part to increased apoptotic signaling with advancing age, increased reactive oxygen species (ROS) production, dysfunctional mitochondrion, and impaired muscle regeneration following injury or disuse.

Implicated in the growth and regeneration of skeletal muscle, muscle stem cells (i.e., satellite cells) show a decreased efficiency in their ability to proliferate and/or differentiate with advancing age $(12 ; 27)$. Normally quiescent, satellite cells are activated in response to injury and/or stress and subsequently proliferate, differentiate, and grow in order to repair damaged muscle tissue $(59 ; 211)$. With increasing age, satellite cell numbers gradually decline, possibly leading to the impaired regenerative ability of muscle observed in many older adults $(12 ; 61)$. Moreover, satellite cell function is perturbed in many myopathies, such as the muscular dystrophies, greatly impairing the maintenance and repair of damaged muscle. Thus, a better understanding of the mechanisms behind satellite cell activation and function is required in 
order to develop novel therapies aimed at reducing skeletal muscle degeneration which occurs in aging and myopathic conditions.

Activation of silent mating type information regulation homolog 1, or Sirtuin 1 (Sirt1), has been shown to be beneficial in neurodegenerative, metabolic, inflammatory, and neoplastic diseases, and has also been shown to increase lifespan in several organisms $(75 ; 102 ; 196)$. Sirt1 appears to play an important role in the regulation of oxidative stress, apoptosis, mitochondrial biogenesis, insulin sensitivity, and satellite cell proliferation (43; 242). Typical regimens of increasing Sirt1 activity levels experimentally include caloric restriction and supplementation of the naturally occurring polyphenol, resveratrol. Our preliminary data indicate that $\mathrm{NAD}^{+}$biosynthesis, Sirt1 activity, mitochondrial biogenesis, and satellite cell function are impaired with aging, and also in aged muscles following a period of disuse (123). Furthermore, nuclear apoptosis also appears to play a role in sarcopenia. Apoptosis, particularly through the mitochondrial pathway, is accelerated in aging and disuse. However, the role of Sirt1 and its implications in the treatment of sarcopenia and muscle regeneration have not been fully established. Recent work from our lab has shown that low levels of resveratrol, a Sirt1 activator, could effectively reduce levels of oxidative stress in hindlimb suspended animals, but was unable to attenuate muscle losses in these animals (123). What is not known is if a higher dose of resveratrol, and therefore increased Sirt1 activation, will provide protection against muscle atrophy following a period of muscular disuse and subsequently improve the regenerative ability of these muscles through the activation of satellite cells. Furthermore, the role of Sirt1 expression in muscle regeneration has yet to be directly examined.

The main objective of this project is to examine the role of Sirt1 expression on skeletal muscle regeneration and to subsequently use this information to improve muscle growth and functional recovery in various physiological conditions. Our central hypothesis is that increasing Sirt1 activity will improve skeletal muscle regeneration (i.e., strength and/or size) following a period of disuse in aged animals, and also in response to an acute cardiotoxin injury. We will 
use a combination of nutritional and genetic approaches to regulate Sirt1 activity in rodent muscles.

\section{Specific aim \#1: Establish the efficacy of resveratrol, a Sirt1 activator, to improve} muscle regeneration after disuse.

Our working hypothesis is that increasing Sirt1 activity through resveratrol supplementation will reduce mitochondrial-associated apoptosis, preserve muscle mass, and increase activation of muscle satellite cells during a period of reloading following disuse in aged rats. This will lead to improvements in muscle morphological and/or functional characteristics.

\section{Specific aim \#2: Define the role of Sirt1 in improving skeletal muscle regeneration}

\section{following injury.}

Our working hypothesis is that Sirt1 will improve muscle regeneration (i.e., strength and/or size) following an acute cardiotoxin injury through an increase in satellite cell activation and proliferation. This will hasten the recovery process of the injured muscles.

\section{Study Limitations}

Each of our two specific aims will have certain a priori limitations that we will have to be mindful of while conducting the research. In our first specific aim we utilize aged animals, which present a unique set of problems when attempting to examine muscle regeneration. It is well known that aged organisms have a reduced capacity to regenerate bodily tissues, due to decreased functional abilities of their intrinsic stem cells or in the environment in which these stem cells reside. Regardless of the reason, the potential conflict with this diminished regenerative ability is that it can be difficult to determine if a given intervention is effective, as any benefits will be much less noticeable than in a younger organism. Thus, even if resveratrol appears to be beneficial in our model, the blunted regenerative response of the aged animals 
may prevent any of these benefits from becoming statistically significant. Another potential problem that we may have with these animals is their age-associated increase in morbidity and mortality. It is possible that some of these animals will die during the course of the study or develop systemic conditions which could unintentionally affect our findings. However, it is important that we use aged animals in this study as it is the aging human population which is more susceptible to similar periods of disuse and immobilization, such as extended hospital stays, and for whom there needs to be novel therapies developed to enable more efficacious recoveries.

The limitations of our second specific aim are less visibly obvious. In analyzing the muscle regeneration of our transgenic animals, we are only concerned about the effects of Sirt1 expression on the general regeneration process. However, the effects of Sirt1 are mediated by its activation of several downstream targets. This being the case, we are only attempting to define the general role of Sirt1 in regeneration, and will not attempt to examine each individual downstream target to determine which specific pathway may be inducing these potential effects. This is important, as it must first be established whether Sirt1 expression has any effects on skeletal muscle regeneration before attempting to distinguish which pathways Sirt1 is exerting its influence upon to initiate these effects. Thus, if our results from this study indicate that increasing Sirt1 expression is beneficial to muscle regeneration as hypothesized, future studies could then be directed toward deciphering these individual pathways and proteins which are recruited and enable the beneficial effects. However, it must be restated that the aim of this current study is attempting only to establish whether Sirt1 expression in general has any significant role in skeletal muscle regeneration. 


\section{Chapter 2}

\section{Background and Significance}

\subsection{Skeletal muscle}

Skeletal muscle comprises $40-50 \%$ of the total mass in the average young adult human. It is crucial for locomotion, proper metabolism, generating heat during periods of cold stress, and the maintenance of posture $(24 ; 131)$. Skeletal muscle fibers are multinucleated and exhibit central nucleation on formation or repair from injury, but these nuclei later migrate to a subsarcolemmal position and are then considered to be myofibers $(51 ; 114)$. The myofiber is the basic structural element of skeletal muscle (234), and is formed from fused myoblasts. The basic functional unit of skeletal muscle is the sarcomere, which lies within the myofiber and contains thin actin and thick myosin filaments which interact with each other upon neuromuscular stimulation (234). These signals, carried by acetylcholine-induced action potentials, release calcium stored in the sarcoplasmic reticulum, causing the actin and myosin to alternately overlap and dissociate, decreasing the size of the sarcomere which is the source of muscle contraction (35; 229).

Myosin composition. Encompassing 25\% of the total skeletal muscle protein pool, myosin is the most abundant protein and consists of multiple myosin heavy chain (MyHC) isoforms (91). Four MyHC isoforms have been identified in small mammals; MyHC type I, MyHC type IIA, MyHC type IIX, and MyHC type IIB myofibers (40). Slow MyHC type I myofibers are resistant to fatigue, fast MyHC type IIA myofibers are moderately fatigue resistant, fast MyHC type IIB myofibers show no resistance to fatigue, and MyHC type IIX myofibers are an intermediate between MyHC type IIA and MyHC type IIB fibers (234). A number of experiments on skinned muscle fibers have established the fiber types as I, IIA, IIX, and IIB (40). Shortening 
velocity and force are highest in type IIB fibers and lowest in type I fibers $(29 ; 30)$. While humans possess the gene for MyHC type IIB myofibers, the corresponding protein does not appear to be expressed $(221 ; 247)$. Thus, human skeletal muscle is only comprised of three pure fiber types (I, IIA, and IIX), and two additional hybrid types (I-IIA and IIAX) (28; 225; 250; 251). While skeletal muscle normally maintains a constant protein turnover with little physiologic impact, conditions such as aging, disease, or disuse may tilt this balance to favor catabolism, producing a variety of complications.

\subsection{Sarcopenia}

Sarcopenia is a multifactorial, involuntary loss of skeletal muscle mass and function (e.g. strength) which occurs as a consequence of aging $(36 ; 67 ; 246)$. Up to $15 \%$ of individuals over the age of 65 and as much as $50 \%$ of those over the age of 80 are thought to suffer from sarcopenia (15). Furthermore, it was estimated that the direct cost of healthcare attributed to sarcopenia in the United States in 2000 was $\$ 18.5$ billion (127). Currently, the primary cause of sarcopenia has not been well established. However, as the definition implies, a multitude of factors have been implicated in reducing the quality of life and functionality of those afflicted with this condition.

Muscle fiber and strength decline. Defined as the maximal tetanic force $\left(P_{0}\right)$ produced per muscle fiber cross-sectional area (CSA), specific force $\left(\mathrm{P}_{0} / C S A\right)$ is a widely used measure of relative muscular strength. It has been shown that muscles in elderly individuals produce a lower specific force than do young control subjects $(70 ; 141)$. In general, muscle strength gradually decreases $12 \%-15 \%$ per decade beyond the age of 50 , with a larger decrease seen after the age of $65(152 ; 167 ; 177)$. Furthermore, there is a preferential loss of the larger diameter type II fibers as compared to type I fibers with increasing age, which may be caused by a decrease in high-intensity activities needed to stimulate these fibers (241). The 
loss in muscle mass may also be due to an increase in apoptotic signaling that is seen in older adults $(144 ; 165 ; 193)$. Subsequently, these two variables lead to an approximately $50 \%$ decline in the number of total muscle fibers and a $30 \%$ loss in muscle mass between the ages of 20 and $80(81 ; 87)$. Therefore, it may not be surprising that sarcopenia has been shown to be predictive of falls (46), and also leads to increased mortality due to diminished muscle strength $(168 ; 180)$.

Additional factors. In older age, fast motor units become denervated and subsequently reinnervated with slow motor units, leading to increased muscle fatigability during periods of high-intensity work (85). In moderately active 75-year old males, sarcopenia may account for reductions in fiber pennation angle, maximal voluntary force output, and relaxation rate of the muscle $(126 ; 132 ; 178)$. Hormonal factors may also play a role in the development of sarcopenia. Growth hormone (GH), insulin-like growth factor (IGF-1), and androgens, which function in the growth and development of skeletal muscle, all appear to decrease with older age (36).

\section{$\underline{2.3 \text { Mitochondrial Function and Apoptosis }}$}

Another factor that appears to be detrimental to skeletal muscle and contribute to nuclear apoptosis are dysfunctional mitochondrion. Mitochondria, deemed the "powerhouse of the cell”, are crucial for producing adenosine triphosphate (ATP), a ready-usable energy substrate for multiple biological processes (140).

The volume density of mitochondria is fundamental in the ability to sustain a steady-state of muscular work (48). Several studies have shown that mitochondrial content decreases with age $(63 ; 111 ; 166 ; 203 ; 216 ; 248)$. This may be due in part to a reduction in several mRNA transcripts for genes encoding mitochondrial proteins $(13 ; 38 ; 248)$. Furthermore, there appears to be a decrease in the function of these remaining mitochondria $(22 ; 140 ; 187 ; 191 ; 216)$. 
However, it is interesting to note that a study by Rasmussen et al. (197) concluded that the reduced mitochondrial capacity is associated with reduced exercise, and is not due to chronological age. Energy defects stemming from impaired or dysfunctional mitochondria have been implicated in forms of movement disorders, deafness, blindness, dementias, cardiomyopathy, myopathy, renal dysfunction, neurodegenerative disorders, metabolic disorders, cancer, and aging $(76 ; 244)$. Regardless of the cause, it is clear that with aging comes a reduction in the total number and functionality of mitochondria, possibly leading to muscular atrophy and impaired regeneration.

Oxidative stress. First described by Denham Harmon, the free radical theory of aging suggested that the production of free radicals as a byproduct of normal mitochondrial respiration would play a major role in the process of aging (103). Recent work has shown that reactive oxygen species (ROS) such as peroxides, which are not free radicals, also cause damage to the cells via oxidative stress (23). Therefore, Harmon's original theory has been revised to include these developments, and is now referred to as the Oxidative Stress Theory of Aging (23). Oxidative stress has thus been defined as the 'imbalance between oxidants and antioxidants in favor of the oxidants, potentially leading to damage (217).

While initial assessments concluded that $2-5 \%$ of oxygen used during respiration is converted to the ROS superoxide anion $(31 ; 155)$, more recent studies have shown that ROS production may be closer to $0.15 \%$ (223). Originally thought to be only harmful to the cell, it has now been shown that ROS are important signaling molecules that function in gene expression as well as cell and tissue homeostasis $(82 ; 125)$. Redox signaling associated with ROS has been associated with maintenance of force production during contractions, insulin signaling, and glucose uptake (124). ROS are also thought to be involved with the mediation of skeletal muscle adaptation due to contractile activity through the activation of redox-sensitive transcription factors $(125 ; 202)$. Furthermore, ROS have been implicated in the activation of multiple signaling cascades. 
Mitochondrial associated apoptosis. Losses in muscle mass due to disuse atrophy and sarcopenia may be attributed to an increase in apoptotic signaling. Apoptosis is a tightly regulated process causing cellular destruction without damaging surrounding tissues nor promoting inflammation (133). It is necessary for a variety of biological processes including cellular turnover, tissue homeostasis, and embryogenesis (133). While the apoptotic response may not target whole fibers, it may result in the selective elimination of individual myonuclei (84). The mitochondrion is considered to be the vehicle through which the integration of apoptotic signaling and the induction of apoptosis is initiated (2). While usually present in organisms as inactive zymogens (procaspases), activated cysteine-aspartic proteases (caspases) are responsible for carrying out the actual cellular decomposition (106). These caspases may be activated through both the intrinsic and extrinsic pathways (71). Of particular relevance is the mitochondrial associated intrinsic pathway of apoptosis. Mitochondria are considered to be the prominent regulator of the intrinsic apoptotic pathway as they house a variety of pro- and anti-apoptotic factors (128). Cytochrome c, a small electron carrier associated with the inner mitochondrial membrane, has been one of the most extensively studied mediators of apoptosis (162). When the proper functioning of mitochondria is inhibited, cytochrome $\mathrm{c}$ is released into the cytoplasm where it associates with apoptotic proteaseactivating factor-1 (Apaf-1), dATP, and procaspase-9, which forms the apoptosome (128). Subsequently, the homo-oligomerization of procaspase- 9 activates caspase-3, in turn initiating apoptosis (128).

Mitochondria also possess a caspase-independent apoptotic pathway. This pathway is carried out by the apoptosis-inducing factor (AIF) as well as endonuclease G (EndoG) (240). AIF is released into the cytoplasm from the mitochondria, translocates to the nucleus, and then binds to DNA to initiate chromatin condensation (72). It may also produce large-scale DNA fragmentation by forming an active DNase with cyclophilin A (39). EndoG, on the other hand, is a mitochondrion-specific nuclease which is required for normal cell proliferation (113). In 
apoptotic situations, EndoG participates in oligonucleosomal DNA fragmentation when it is released from the mitochondria and enters the nucleus (150).

BCL-2 family regulation. Required for the release of pro-apoptotic factors residing in the mitochondria, the mitochondrial outer membrane permeabilization (MOMP) is necessary for initiation of mitochondrial-associated apoptosis (55). The integrity of the outer mitochondrial membrane (OMM) is influenced by the BCL-2 (B-cell lymphoma 2) family of proteins, which are considered either pro- (e.g. Bax, Bid, Bak, Bim) or anti-apoptotic (e.g. BCL-2, BCL- $\mathrm{X}_{\mathrm{L}}$ ) (49). BCL-2 and BCL- $\mathrm{X}_{\mathrm{L}}$ inhibit the activity of Bax and/or Bak under homeostatic conditions (9). It is thought that under apoptotic conditions, Bid and/or Bim, which are necessary for the activation of $\mathrm{Bax}$ and/or Bak, downregulate $\mathrm{BCL}-2$ and $\mathrm{BCL}-\mathrm{X}_{\mathrm{L}}$ activity and therefore initiate apoptosis (56). Furthermore, while Bid can be activated by the cleavage of caspase-8, there can also be cross-talk between the extracellular and intracellular pathways of apoptosis (149).

\subsection{Satellite Cells}

In addition to the problems associated with muscle atrophy due to disuse and immobilization, aged skeletal muscle also displays impaired regeneration. This may be due in part to a reduction in satellite cell abundance with age. Several studies have shown that following sexual maturity, satellite cell numbers begin a gradual descent $(21 ; 50 ; 209)$. First described in 1961 by Alexander Mauro, the satellite cell resides on the periphery of skeletal muscle fibers, between the sarcolemma and basal lamina (163). Satellite cells were first shown to function as myoblasts in 1969 by Reznik, who was also the first to term them stem cells (200). This was further supported by the work of Moss and Leblond, who showed that satellite cells become mitotically active and contribute nuclei to both damaged and growing myofibers $(173 ; 174)$. It was subsequently shown in 1977 that satellite cells, and not myofibers, contribute these nuclei to the regenerating skeletal muscle (222). Satellite cells are therefore very 
important, as they are a major source of skeletal myoblasts that repopulate damaged and growing post-natal skeletal muscle tissues and also have the ability to form new myofibers (17; $59 ; 211)$.

Satellite cell provisions. As a tissue-specific stem cell population, satellite cells must be able to provide differentiating progeny of which to fuse with new and damaged myofibers, and also have a mechanism capable of self-renewing the stem cell population. For these to occur, a subpopulation of satellite cells must be able to stop itself from progressing down the myogenic lineage and revert back into quiescence, or, a subpopulation of these cells must divide asymmetrically, therefore forming both differentiating and stem cell progeny (182). These mechanisms do not have to be mutually exclusive, and it has been suggested that each of these scenarios may occur in vivo $(138 ; 260)$.

Satellite cell replenishment. Upon activation, the majority of satellite cells begin expressing the myogenic regulatory factor MyoD, and upon differentiation, express myogenin $(66 ; 135 ; 260)$. In a study by Zammit et al., it was shown that a subset of activated satellite cells (i.e. MyoD+ ${ }^{+}$switches off the marker while retaining Pax7 expression and returning to a quiescent state (260). This suggests that satellite cells replenish their quiescent pool through the reversal of the myogenic lineage paradigm. However, multiple studies have shown that satellite cells may divide asymmetrically, giving rise to differential daughter cells that help to reestablish the quiescent pool $(62 ; 138 ; 215)$. Furthermore, Shinin et al. suggest that satellite cells are capable of asymmetric division in which one of the cells retains the maternal 'immortal' DNA strand (215), a model proposed by John Cairns in 1975 (37).

In Cairns immortal strand hypothesis, the true stem cell, when dividing, retains the template strand of DNA, while the newly synthesized strand would be inherited by the cell destined for differentiation, therefore reducing the likelihood for a build-up of potentially oncogenic mutations in the stem cell population (37). However, it has been shown that heterogeneity exists within the satellite cell population, with only a portion of cells which can 
undergo asymmetric divisions and retain immortal DNA strands in this manner, suggesting that not all $\mathrm{Pax} 7^{+}$satellite cells on adult myofibers have equivalent lineages $(137 ; 215)$. Further supporting this, it was shown that satellite cells that remain in contact with the basal lamina of the myofiber during asymmetric division will retain their stem cell function, while cells that lose contact with the basal lamina during this period initiate Myf5 expression, which spurs differentiation (138). This suggests that the niche environment of satellite cells plays an important role in their proliferative capacity.

Molecular markers of satellite cells. Satellite cells have been shown to express a wide variety of molecular markers which enable their identification. Among these are the celladhesion protein M-cadherin, CD34, the myogenic regulatory factor myf5, Caveolin-1, Syndecan-3 and 4 , and C-Met $(6 ; 17 ; 64 ; 66 ; 120 ; 243)$. Of greater importance, Pax7 is expressed in the nucleus of all quiescent adult satellite cells and appears to be vital for maintenance of the satellite cell population in adults $(183 ; 211)$. The 'Paired Box', or Pax, family of genes are transcription factors which are essential for embryonic development $(26 ; 159$; 239). There are nine known Pax genes (Pax1-9) in mammals, which are subdivided into four groups based on genomic structure, sequence similarity, and function (245). Forming one of these subgroups, Pax3 and Pax7 are homologous genes, but play different roles during embryological development (182). Pax3, a paralogue of Pax7, is crucial for myogenesis in the developing embryo, demonstrated by Pax3 mutant mice which lack limb musculature (20; 93; 228). However, while both $\mathrm{Pax} 3$ and $\mathrm{Pax} 7$ are important in regulating myogenesis, Pax3 is downregulated before birth, suggesting that its role in adult tissues is limited (156). Supporting this idea, it has been shown that most of the function of Pax3 can be replaced by Pax7 after birth (199). Furthermore, Pax7-null mice progressively lose satellite cells in adulthood due to apoptosis, whether Pax3 is expressed or not, which implies that Pax3 is not able to replace Pax7 in vivo for the maintenance of satellite cell viability during adulthood (199). Also, it has been shown that $\mathrm{Pax}^{-/-}$mutant mice lack satellite cells and fail to grow or regenerate after birth, 
subsequently dying within $2-3$ weeks $(160 ; 211)$. Moreover, multiple studies have shown that Pax7 is required for normal muscle regeneration, and regeneration is severely retarded in its absence or depression $(147 ; 207)$.

Activation of satellite cells. Following injury, multiple signals appear to activate satellite cells, which subsequently begin proliferating on the second day post-injury (57). Satellite cell activation requires sphingosine-1-phosphate on the inner side of the plasma membrane, and without its synthesis, satellite cells do not enter the cell cycle and regeneration is incomplete (176). The production of nitric oxide (NO) through increased NO synthase (NOS) activity may also be important for satellite cell activation through activation of matrix metalloproteinases, which induce the release of hepatocyte growth factor (HGF) from the extracellular matrix (232). In turn, HGF binds to its receptor on satellite cells, c-Met, thus stimulating an activated satellite cell response (231).

Differentiation of satellite cells. The switch from satellite cell proliferation to differentiation appears to be under the control of notch and Wnt signaling. Following injury, sustained notch signaling is necessary for the expansion of the satellite cell progeny, while canonical Wnt signaling subsequently drives differentiation $(32 ; 61 ; 62)$. Defective notch signaling inhibits the self-renewal and proliferative properties of satellite cells, while notch signaling enhancement leads to increased regeneration in aged muscle $(61 ; 138)$. Increased Wnt signaling, on the other hand, appears to drive the differentiation of proliferating myoblasts (32). The timing of this is crucial, as premature Wnt signaling leads to early differentiation and fusion of the myoblasts before the number of proliferating myoblasts are sufficient for proper regeneration (32). However, the signaling of Wnt during muscle regeneration appears to be complex. Acting through a non-canonical Wnt pathway and upregulated during regeneration, Wnt7a is necessary for the expansion of the satellite cell population during regeneration (142). Opposing the normal functions of Wnt signaling, Wnt7a does not affect the growth or 
differentiation of myoblasts (57). While overexpression of Wnt7a enhances regeneration, muscles lacking Wnt7a display a reduction in satellite cell number following regeneration (142).

MyoD expression. Moving forward through the satellite cell regenerative process, the MyoD family regulators of muscle determination and differentiation (MRFs) are expressed in committed myogenic precursor cells. Among these regulators are MyoD, Myf5, Mrf4, and myogenin (57). In a study using a single-cell RT-PCR assay, activated satellite cells expressed both MyoD and Myf5 together, myogenin was expressed later in these cells, and many cells expressed all four MRFs simultaneously (66). However, MyoD may be the most crucial regulator in the regenerative process. In MyoD null satellite cells, myogenin is reduced and Mrf4 is totally absent, resulting in a severely blunted differentiation capacity (65). Furthermore, in the absence of MyoD, there is a marked impairment in the regenerative ability of muscle due to perturbations in cell proliferation and differentiation levels (164).

Myogenin expression. Lastly, skeletal muscle regeneration is aided through the expression of myogenin and MRF4. While neither are required for satellite cell development or maintenance (90), the induction of myogenin is required for the formation of myotubes and fibers (143). This was observed in mice with a post-natal recombination of myogenin which displayed reduced muscle mass, suggesting that the absence of myogenin prevented the skeletal myoblasts from contributing to post-natal muscle growth (134). Thus, the induction of myogenin commits the cells to differentiate and subsequently grow.

\subsection{Muscle regeneration}

While the degenerative and regenerative phases involved in skeletal muscle regeneration are essentially the same, the kinetics of these phases may be influenced by factors such as the severity of injury, the muscle injured, and also the animal model used (119; $145 ; 170 ; 188)$. To develop methods of studying muscle regeneration, the design of animal 
models that are controlled and reproducible is necessary. One of the easiest and most reproducible methods of inducing injury is the use of myotoxins, such as cardiotoxin (CTX), notexin (NTX), or bupivacaine $(69 ; 96 ; 104)$. Injected directly into the individual muscle of interest, these toxins have a wide range of biological activities and exert their effects in a very short manner. CTX, a peptide isolated from snake venom, is a protein kinase C-specific inhibitor that appears to disrupt membrane organization, induce the depolarization and contraction of muscle cells, and will lyse various cell types (51). Typically with this model, muscle degeneration is seen within a day of injection, myogenic cell differentiation is seen $\sim 5-6$ days later, and normal histologically and morphologically mature muscle is restored after $\sim 3-4$ weeks following the injury (51). These rapid results, paired with a high degree of reproducibility, make the CTX model an excellent method for studying muscle regeneration.

Models of muscular disuse. While the CTX protocol works very well, disuse and/or immobilization models may be more physiologically relevant for studying muscle regeneration. Actual muscle loss varies in relation to the mode of disuse/immobilization. In the large leg muscles of humans, a head down bed rest position induces the greatest decreases in fiber CSA $(5 ; 8 ; 19 ; 34 ; 74)$, with slightly smaller decreases seen following casting $(109 ; 110 ; 129)$, followed closely behind by unilateral leg suspension and knee immobilization $(45 ; 73 ; 78 ; 257)$. Also, it has been shown that muscle fibers experience greater losses in CSA than do whole muscles $(11 ; 109 ; 238 ; 257)$. The common element binding these various modalities has been suggested to be due to both a reduction in net protein synthesis and also an increase in protein degradation $(195 ; 235)$. While slow type I fiber muscles experience greater atrophy than do fast type II fiber muscles in rodent models $(25 ; 83 ; 97 ; 121)$, in humans this may not be true $(11$; $238 ; 257)$.

Hindlimb suspension. A widely used model of disuse for studying muscle atrophy and regeneration is hindlimb suspension (HS). Originally developed by NASA in order to study the effects of spaceflight in a ground-based model, $\mathrm{HS}$ is a common technique used to study a 
variety of skeletal muscle adaptations in response to disuse $(100 ; 181 ; 201 ; 204 ; 233 ; 235)$.

Generally, a rodent is suspended by its tail so that the hind limbs cannot reach the cage floor, but the animal is still free to move around its cage and groom itself (100). A suspension period of fourteen days is typically sufficient to induce significant decreases in muscle mass in rodents $(100 ; 218 ; 220 ; 252 ; 258)$. Due to decreased signaling to the muscles, HS may inhibit mitochondrial biogenesis and/or increase apoptotic signaling (190). Furthermore, HS has been shown to elicit a fiber type switch from slow to fast MyHC isoforms in response to the period of disuse $(192 ; 224 ; 230)$. To study the regenerative potential of the atrophied myofibers, a subsequent reloading period may be incorporated immediately following HS. The HS protocol combined with this reloading period is very similar to the atrophy and regeneration of muscles in humans who undergo periods of immobilization such as bed rest. This is noteworthy, as 34\%$50 \%$ of hospitalized elders undergo a decline in their functional status during the period between admission and discharge (118). Thus, the HS model is a physiologically relevant paradigm to study the response of muscles to disuse in populations such as the elderly, who are increasingly prone to hospitalizations with advancing age $(1 ; 98 ; 169)$.

\section{$\underline{2.6 \text { Sirtuin } 1}$}

Resveratrol as a Sirt1 activator. Resveratrol (3,5,4'-trihydroxystilbene) is a naturally occurring phytoalexin that is found in high concentrations in the skin of red grapes, but is also found in mulberries, peanuts, pines, and root extracts of the weed Polygonum Cuspidatum (10; 116). The function of this compound in plants is to provide protection from fungal infections (146). It consists of two isomers, trans-resveratrol and cis-resveratrol, of which the trans isomer is the more stable (213). Trans to cis isomerization is induced by both UV light and high $\mathrm{pH}$, while cis to trans isomerization is facilitated by high temperature, low $\mathrm{pH}$, or visible light (213). Resveratrol has a low biological availability however, as only $\sim 20 \%$ of available resveratrol in 
the intestines of rats is absorbed (213). Resveratrol has been shown to have anti-inflammatory properties, and its cardioprotective qualities have been implicated in the 'French paradox', the observation that French people suffer from a relatively low incidence of coronary heart disease despite a diet high in saturated fats $(86 ; 102 ; 122 ; 196)$. It has also been shown to have anticancer properties, due mainly to its cell cycle control and by initiating apoptosis in transformed cells (75). Lastly, it has been shown to be beneficial to reduce oxidative stress in skeletal muscle as well (122; 123; 206).

Metabolic benefits. Resveratrol appears to impart improvements in skeletal muscle metabolism. It improved the health and survival of animals placed on a high fat diet, and protected them from metabolic disease (16; 139). In a 2006 study by Lagouge et al., resveratrol treated mice placed on a high fat diet gained significantly less weight than non-treated mice placed on the same high fat diet, and also had much lower body fat percentages than the nontreated animals (139). The resveratrol treated mice had body weights and body fat percentages similar to a control group of standard chow diet mice. These beneficial effects may have been due to the observed increases in basal energy expenditure and thermogenesis of the resveratrol treated mice in the study (139). In a similar study by Baur et al., and using a lower dose of resveratrol than the Lagouge study, resveratrol supplementation did not significantly improve body weights of the treated animals (16). However, in both the Baur and Lagouge studies, resveratrol treated mice were protected from insulin resistance, which had lower circulating levels of insulin and improved glucose tolerance compared to the non-treated mice put on a high fat diet $(16 ; 139)$. Subsequent studies have further suggested that resveratrol supplementation may stimulate glucose uptake in skeletal muscle independent of insulin, as well as increasing insulin-dependent glucose transport $(54 ; 77 ; 186)$. Furthermore, resveratrol has been shown to improve glucose tolerance in diabetic rats $(54 ; 226)$. These findings are noteworthy, as it is well known that muscle regeneration is impaired in certain metabolic disorders such as diabetes (112). 
Previous findings. Recent work from our lab has also supported the role of resveratrol in reducing oxidative stress. In a 2010 study by Ryan et al., resveratrol administration significantly lowered levels of oxidative stress in aged mice (206). In this study, mice were pretreated with a $0.05 \%$ trans-resveratrol chow diet for ten days. At day seven they were subjected to twenty maximal electrically-evoked isometric contractions. This was repeated once more each of the next two days, whereupon the animals were sacrificed. The results of this experiment showed that resveratrol supplementation attenuated the exercise-induced increase in xanthine oxidase activity, lowered hydrogen peroxide $\left(\mathrm{H}_{2} \mathrm{O}_{2}\right)$ levels, and increased the ratio of reduced glutathione to oxidized glutathione in exercised muscles (206). Furthermore, resveratrol increased the levels of both catalase and MnSOD in the exercised muscles.

In another 2010 study from our lab, Jackson et al. studied the effects of resveratrol on hindlimb suspended rats (123). These animals were pretreated for 7 days with rat chow containing $0.05 \%$ trans-resveratrol, which corresponded to a dose of $12.5 \mathrm{mg} / \mathrm{kg} / \mathrm{day}$. Following the pretreatment phase, the animals were suspended for a period of fourteen days. Resveratrol was able to attenuate the disuse-induced decrease in plantar flexor isometric force in the aged animals, and also increased catalase activity, MnSOD activity, and MnSOD protein content in the gastrocnemius muscles of these animals. It was also able to decrease $\mathrm{H}_{2} \mathrm{O}_{2}$ and lipid peroxidation levels in the muscles of the old animals. This is important, as it has been suggested that reduced levels of oxidative stress are associated with faster muscle regenerative capabilities (259). In a subsequent study by Jackson et al., it was demonstrated that long-term (10 month) resveratrol supplementation increases MnSOD and reduces both $\mathrm{H}_{2} \mathrm{O}_{2}$ and lipid peroxidation levels in aged mice (122). It is interesting to note that while resveratrol was able to modulate oxidative stress levels in the study, it was unable to attenuate sarcopenia in the aged mice. It is possible that the potential muscle-sparing benefits of resveratrol were not observed due to a lack of any significant muscle perturbation in these mice. Ex vitro physiological analyses revealed no significant differences in muscle twitch properties, twitch/tetanus ratio, and 
a fatigue index protocol in the resveratrol treated mice compared to the non-treated mice (122). However, resveratrol produced a rightward shift in a force frequency curve, which is suggestive of a preservation of the fast muscle phenotype with suspension. It is currently unknown if this fast muscle preservation will translate into an improved muscle regenerative capacity.

Resveratrol and muscle atrophy. In vitro, resveratrol has been shown to inhibit protein degradation and attenuate atrophy of skeletal muscle fibers in several studies $(205 ; 254 ; 255)$. In vivo, these actions are not as well studied. However, a recent study by Momken et al. examined some of the effects of resveratrol on skeletal muscle atrophy and function following a period of hindlimb suspension (171). In this study, soleus muscle atrophy was significantly attenuated with resveratrol supplementation. Also, resveratrol was able to ease the losses in type I fiber area following suspension. However, resveratrol was not able to ameliorate maximal tetanic force $\left(P_{0}\right)$ losses observed following the suspension period. Presently, there are no studies that examine the role of resveratrol in skeletal muscle regeneration.

Sirt1 in skeletal muscle. While resveratrol has been shown to produce beneficial effects in a variety of studies, it is thought that much of this is due to its ability to activate Sirt1. Sirt1, the mammalian ortholog of yeast Sir2, was initially implicated as an important regulator of life span in this organism $(130 ; 236)$, and subsequently in higher eukaryotes (154). Sirt1 belongs to the family of sirtuins (Sirt1-7), the class III protein deacetylases which use $\mathrm{NAD}^{+}$as a cofactor in which their activity is regulated by $\mathrm{NAD}^{+} / \mathrm{NADH}$ ratios that change with respiratory activity (185). Sirt1 may be activated in vivo through the aforementioned resveratrol supplementation protocol, a controlled regimen of caloric restriction (58), and with exercise (136). However, recent evidence has suggested that Sirt1 is not directly activated through resveratrol $(18 ; 184)$, but instead functions indirectly through the actions of AMP-activated protein kinase (AMPK) $(41 ; 105)$. Nevertheless, Sirt1 plays a varied physiologic role, regulating the activity of transcription factors and co-regulators which are involved in cell growth, metabolism, differentiation, survival, apoptosis, and the inflammatory or stress response (185). 
While Sirt1 has been shown to play a protective role in age-associated pathologies such as diabetes, cancer, neurodegenerative disorders, and cardiovascular disease (108), its role in skeletal muscle regeneration and aging remains to be established.

PGC-1 activation. The beneficial effects of Sirt1 on skeletal muscle metabolism are mainly due to its ability to deacetylate and activate the PPAR gamma coactivator 1, PGC-1 (185). Expression of PGC-1 $\alpha$ in myotubes increases the expression of genes in the respiratory chain and enhances mitochondrial biogenesis by increasing the activity of the nuclear respiratory factor NRF1 and its target, mtfA, which in turn activates mitochondrial DNA replication (253). PGC-1a is very important in oxidative metabolism, and the expression levels of both PGC-1 $\alpha$ and Sirt1 are higher in the slower type I oxidative fibers as compared to the fast type II glycolytic fibers (151). Under conditions of starvation, PGC-1a is responsible for inducing the switch in mitochondrial metabolism from glucose to fatty acid oxidation (92). In vitro, Sirt1 has been shown to deacetylate PGC-1 $\alpha$ (179), and is required for the fasting induced deacetylation of PGC-1 $\alpha$ in skeletal muscle (92). As AMPK may be an upstream regulator of Sirt1 $(41 ; 105)$, its inhibition prevents the deacetylation of PGC-1a by Sirt1 in response to glucose deprivation and exercise, therefore blocking the subsequent switch to fatty acid metabolism $(41 ; 42)$.

Sirt1 appears to play a complicated role in skeletal muscle regeneration that is not yet fully understood. It has been suggested that Sirt1 may alter the lineage/cell fate decisions of stem cells by sensing redox status $(157 ; 161)$. In a 2003 study by Fulco et al., in vitro analysis revealed that Sirt1 overexpression repressed the muscle transcriptional factor MyoD (89). This in turn blocked several transcripts, including those encoding myogenin, thereby inhibiting the fusion of myoblasts into myotubes. However, reducing Sirt1 levels by RNA interference resulted in the differentiation of myoblasts in these cultures (89). The mechanism responsible for the inhibition of myogenesis is attributed to the recruitment of Sirt1 to muscle regulatory regions on chromatin through its interaction with a MyoD/PCAF complex and the histone acetyltransferase 
GCN5, controlling the myogenic factor MEF2, and ultimately repressing the myogenic gene program (185). Moreover, the $\mathrm{NAD}^{+}$to $\mathrm{NADH}$ ratio and the redox state are closely linked to nutrient availability in muscle cells (242). In a 2008 follow-up study by Fulco et al., cultured myoblasts exposed to glucose restriction showed enhanced Sirt1 activity through an AMPKdependent regulation of Nicotinamide monophorybosiltransferase (NAMPT), thereby preventing their differentiation into myotubes (88).

Sirt1 and insulin-like growth factor 1 (IGF-1) share downstream signaling targets in skeletal muscle, such as forkhead box-O transcription factors (FOXOs)(242). Locally acting IGF-1 has been shown in numerous studies to impart great regenerative potential in both injured skeletal and cardiac muscle tissues $(14 ; 175 ; 189 ; 210)$. However, it has been shown that circulating IGF-1 levels counteract Sirt1 activity (58). Likewise, levels of circulating IGF-1 were reduced during a regimen of caloric restriction (115). Therefore, it appears that Sirt1 and circulating IGF-1 play opposing roles in longevity (153).

Does Sirt1 have a role to play in muscle regeneration? While many beneficial effects have been observed with Sirt1, either directly or through its activation via resveratrol supplementation and/or caloric restriction, little is known about its role in skeletal muscle regeneration. It remains to be established whether increased Sirt1 activity will improve satellite cell activation and function, thereby aiding muscle regeneration following injury or disuse. Therefore, the purpose of this study is to elucidate the role of Sirt1 in skeletal muscle regeneration through its actions on satellite cells. We postulate that increasing the activity of Sirt1 in skeletal muscle will enhance regeneration following disuse or injury, due to enhanced satellite cell functioning, reduced mitochondrial-associated apoptosis, and a preservation of muscle mass and function following a period of disuse. We will use both nutritional and genetic approaches in combination with models of disuse and injury to accomplish our aims. Establishing the efficacy of Sirt1 in the regeneration of skeletal muscle may hold implications for 
future therapeutic use in the treatment of sarcopenia as well as a variety of muscular pathologies. 


\title{
Chapter 3
}

\section{Effects of resveratrol on disuse associated loss and hindlimb reloading in the plantaris muscle of aged rats}

\author{
Brian T. Bennett and Stephen E. Alway
}

Laboratory of Muscle Biology and Sarcopenia, Division of Exercise Physiology, West Virginia University School of Medicine, Morgantown, WV. 


\section{Abstract}

Introduction: Aging is associated with an impaired skeletal muscle regenerative ability following perturbations such as extended periods of hospitalization and other forms of muscular disuse. This may be due in part to a decrease in the total number and function of satellite cells, which are a major source of skeletal myoblasts which repopulate damaged and growing postnatal skeletal muscle tissues. Resveratrol (3,5,4'-trihydroxystilbene) is a naturally occurring phytoalexin which has been shown in skeletal muscle to improve oxidative stress levels and provide protection against disuse-induced muscle atrophy. Therefore, we hypothesized that resveratrol supplementation would significantly improve muscle regeneration during a reloading period immediately following disuse.

Methods: For this study, a total of thirty-six male Fisher $344 \times$ Brown Norway rats (32 mos.) were treated with either a water vehicle or resveratrol via oral gavage. Animals underwent hindlimb suspension (HS) for a period of fourteen days, whereupon they were either sacrificed or allowed an additional fourteen day period of normal ambulation, the reloading period $(R)$. A total of six rats from each the vehicle and resveratrol treated groups were used for the HS and R protocols. Furthermore, two groups of six vehicle treated animals maintained normal ambulation throughout the experiment, and were used as control animals for the HS and R groups. The extent of satellite cell involvement in the regeneration of the plantaris muscles of the $R$ animals was then examined to determine if the resveratrol treatment would prove beneficial.

Results: HS elicited decreases in both body and muscle weights of all of the animals. However, resveratrol supplementation was unable to attenuate these decreases. Resveratrol was able to attenuate decreases in type IIA MyHC fiber CSA following HS, and type IIA and IIB 
fibers following R. Although resveratrol had no effect on the proportion of type I MyHC fibers following either HS or R, it significantly increased the proportion of type IIA fibers following HS. Lastly, although resveratrol could not protect the muscles from increased apoptosis as evidenced by TUNEL staining, it was able to alter apoptotic signaling as shown by an increase in anti-apoptotic Bcl-xL protein abundance following HS.

Discussion: Resveratrol supplementation has previously been shown to be beneficial to soleus muscles undergoing periods of disuse. However, it is unknown if resveratrol can improve muscle recovery following this period of disuse. Utilizing a relatively low dose (125 $\mathrm{mg} / \mathrm{kg}$ ) of resveratrol, we observed benefits to muscle fiber CSA, fiber type distribution, and apoptotic signaling in our aging model of muscle recovery following disuse. It is possible that a higher dose of resveratrol may be able to maximize these benefits. Therefore, further research into this area should be undertaken in order fully elucidate the efficacy of resveratrol in improving skeletal muscle recovery following a period of disuse. 


\section{Introduction}

Skeletal muscle comprises $40-50 \%$ of the total mass in an average young adult human and is crucial for locomotion, metabolism, generating heat, and the maintenance of posture (24; 131). However, the natural process of aging induces an involuntary loss in muscle mass and function, due to the multifactorial condition commonly referred to as sarcopenia $(36 ; 68 ; 246)$. It is thought that as much as $50 \%$ of the population over the age of 80 suffers from this condition (15). Sarcopenia has been shown to be predictive of falls (46), possibly leading to extended hospital stays and periods of immobilization. This is noteworthy, as aging muscles display an impaired recovery following immobilization-induced muscle atrophy $(47 ; 158 ; 227) 26)$. One prominent factor which may prime this impaired recovery is the decline in satellite cell function observed with advancing age $(44 ; 50 ; 61)$.

First described by Alexander Mauro in 1961, the satellite cell resides on the periphery of skeletal muscle fibers between the sarcolemma and basal lamina (163). Normally quiescent, these cells are a major source of skeletal myoblasts which repopulate damaged and growing post-natal tissue while also having the ability to form new myofibers $(17 ; 59 ; 211)$. In response to sufficient perturbations in muscle homeostasis (e.g., muscle injury), satellite cells are activated, proliferate, and differentiate before committing to a myogenic lineage and repairing the tissue. However, aging appears to induce functional limitations in this essential cell population. Several studies have suggested that there is a delayed response to activation cues in aged muscles and their proliferative expansion is initially impaired as well $(12 ; 27 ; 61 ; 214)$. Moreover, in vitro studies have shown an impaired differentiation capacity in aged satellite cells $(50 ; 60)$. It thus appears likely that the culmination of these defects lead to the reduced regenerative faculties in the elderly, reducing both the quality of life and functional independence of this population. Therefore, developing therapies to counteract the inadequate satellite cell response to skeletal muscle insults in the aging population is of utmost importance. 
Resveratrol (3,5,4'-trihydroxystilbene) is a naturally occurring phytoalexin most notably found in the skin of red grapes $(10 ; 116)$. Thought to exert its effects primarily through the activation of the class III protein deacetylase Sirtuin 1 (Sirt1), resveratrol has been shown to benefit oxidative stress levels and also has anti-inflammatory, anti-cancer, and cardioprotective properties $(75 ; 102 ; 122 ; 123 ; 196 ; 206)$. Resveratrol has been shown to inhibit protein degradation and attenuate atrophy of skeletal muscle fibers in several in vitro studies $(205 ; 254$; 255). In vivo, the effects of resveratrol on skeletal muscle atrophy and growth are not well established. A recent study by Momken et al. (172) examined several effects of resveratrol on skeletal muscle during a period of unloading in rats. Using a relatively high dose (400 $\mathrm{mg} / \mathrm{kg} /$ day) of resveratrol, whole body net protein synthesis was maintained following hindlimb suspension, and resveratrol also attenuated soleus muscle atrophy and cross-sectional area (CSA) decreases following suspension. This high dose of resveratrol also maintained tetanic force and twitch tension (172), demonstrating its potential to preserve muscle function following periods of disuse. These beneficial effects of resveratrol on muscle force have also been observed in several other studies $(80 ; 122 ; 139 ; 212)$. Moreover, a recent study determined that resveratrol supplementation in vitro could inhibit dexamethasone-induced increases in the expression of the atrophy-related MuRF1 and atrogen-1 ligases, as well as attenuating increases in protein degradation (3).

While these and a multitude of other studies have examined the effects of resveratrol on various physiological measures in skeletal muscle, no studies to date have directly explored the relationship between resveratrol supplementation and muscle regeneration in aged animals. It seems plausible, given the multiple benefits of resveratrol observed in skeletal muscle, that it may also improve muscle recovery following a period of disuse. We therefore hypothesized that resveratrol supplementation would improve skeletal muscle regeneration/recovery (i.e. muscle mass and/or function) during a period of reloading immediately following disuse. Such an observation is important because muscles from aged animals recover very little mass or 
function after disuse (26). Our data showed that low dose resveratrol has little benefit during the unloading period, but it has some utility for improving muscle recovery after disuse in old rats. The findings of our study may potentially be applied to the development of novel therapeutic treatments to improve muscle recovery in individuals subjected to extended periods of disuse, such as the elderly following lengthy hospitalizations. 


\section{Methods}

Animals. For this study, thirty-six male Fisher $344 \times$ Brown Norway rats were obtained from the National Institute on Aging (NIA) colony that is housed at Harlan (Indianapolis, IN). All of these animals were approximately 32 months of age. The animals were housed at $20^{\circ} \mathrm{C}$ in barriercontrolled conditions under a 12:12 hour light-dark cycle. Proper animal care standards were followed by adhering to the recommendations for the care of laboratory animals as advocated by the American Association for Accreditation of Laboratory Animal Care and by following the policies and procedures detailed in the Guide for the Care and Use of Laboratory Animals as published by the U.S. Department of Health and Human Services and proclaimed in the Animal Welfare Act (PL89-544, PL91-979, and PL94-279). All experimental procedures carried approval by the Institutional Animal Care and Use Committee from the West Virginia University.

Hindlimb suspension. The animals were randomly assigned to either ambulatory cage control (CC; $n=12)$, hindlimb suspended (HS; $n=12)$, or reloaded $(R ; n=12)$ groups. The hindlimb suspension lasted fourteen days as previously described (194). Briefly, orthopedic tape was applied along the proximal one-third of the animals' tail and then placed through a wire harness attached to a fish swivel. This was fixed to the top of a specially designed hindlimb suspension cage which provided the rats with $360^{\circ}$ of movement around the cage. The forelimbs maintained contact with a grid pattern floor, allowing the animals to move, groom themselves, and obtain food and water freely. The exposed tip of the tail was closely monitored to ensure that it remained pink, indicating that HS did not interfere with blood flow to the tail. The suspension height was monitored daily and adjusted to prevent contact between the hind limbs and any supportive surface of the cage. Care was taken so that the suspension angle of the torso of the animals to the cage floor did not exceed $30^{\circ}$. All animals were weighed prior to and following the suspension period to determine total body mass changes. Furthermore, the $\mathrm{R}$ animals were 
released from suspension after the fourteen day protocol and provided an additional fourteen days of normal ambulation, at which point they were weighed and sacrificed.

Nutritional treatment. Beginning one week prior to suspension, eighteen of the rats received $1 \mathrm{ml}$ of $0.1 \%$ Carboxymethylcellulose dissolved in distilled water (vehicle treated), and the remaining eighteen rats received $1 \mathrm{ml}$ of trans-resveratrol suspended in $0.1 \%$ Carboxymethylcellulose dissolved in distilled water (resveratrol treated), administered via oral gavage. Previous studies in our lab $(122 ; 123)$ using resveratrol $(12.5 \mathrm{mg} / \mathrm{kg} / \mathrm{day}$ in the diet or $50 \mathrm{mg} / \mathrm{kg} /$ day by oral gavage) were too low to effectively preserve muscle mass. Therefore, in this study resveratrol was administered at a dose of $125 \mathrm{mg} / \mathrm{kg} / \mathrm{day}$ in this study in order to increase the likelihood of detecting the effects of this compound on muscle wasting and regeneration. This provided a bolus increase in resveratrol every 24 hours. Additionally, the resveratrol treated animals received an identical diet to the vehicle treated animals, but their chow also contained $0.05 \%$ resveratrol. This provided a mechanism to provide a more consistently high level of resveratrol throughout the day. Food and water was provided ad libitum.

Bromodeoxyuridine administration. Immediately following suspension, each of the R animals received a 200mg Bromodeoxyuridine (BrdU; 5-bromo-2'-deoxyuridine) pellet (21-day release; Innovative Research; Sarasota, FL) in order to analyze cell proliferation, as BrdU is a thymidine analog which is incorporated into nuclei during DNA synthesis. Upon release from suspension, a small incision was made in the upper back of anesthetized animals whereupon the BrdU pellet was inserted subcutaneously. The incision was closed with a wound clip (MikRon Autoclip 9mm; Becton Dickinson; Sparks, MD) and the animals were allowed to recover from anesthesia before being returned to their cage. 
Research design. Muscle data was obtained from both the resveratrol and vehicle-treated animals. In addition, two groups of ambulatory non-suspended cage control (CC) animals were used. Six CC animals were examined 21 days after the initiation of the study. These animals acted as controls for the HS groups. A second group of six CC animals were examined 35 days after the initiation of the study and were used as controls for the R groups. Among the suspended animals, six per diet group (i.e., vehicle or resveratrol) were sacrificed 21 days after the initiation of the study (HS) and another six animals per diet group were sacrificed 35 days after the initiation of the study (R). The six HS CC and six R CC animals were also sacrificed at these respective time points.

Animal sacrifices and tissue collection. At the end of the 21 and 35 day experimental periods for the HS and R groups respectively, the animals were sacrificed while under deep anesthesia (5\% Isofluorane/95\% Oxygen). The soleus, gastrocnemius, and plantaris muscles were excised from both hind limbs, blotted to remove excess fluid, and individually weighed. The weight of each muscle was normalized to the animals' body weight and subsequently compared to determine if resveratrol preserved muscle mass. A block obtained from the midbelly of the muscle was embedded in optimal cutting temperature (OCT) compound, flash frozen in liquid nitrogen cooled isopentane, and stored at $-80^{\circ} \mathrm{C}$ until later analyses. Once the muscle groups had been removed, blood samples were obtained from the left ventricle of each animal via a stainless steel needle (19G11/2; Becton Dickinson; Rutherford, NJ). The plasma was then measured (Protea Biosciences Group, Inc.; Morgantown, WV) to determine the concentration of resveratrol in each of the animals. Following blood collection, the animals were immediately euthanized by removing the heart with surgical scissors.

Sirt1 activity. Sirt1 activity was measured using components of the Sirt1 Fluorometric Drug Discovery Kit (BML-AK555; Enzo Life Sciences Inc.; Farmingdale, NY). Briefly, endogenous 
Sirt activity was measured in total plantaris muscle homogenates using the fluorescent deacetylase substrate and developer provided in the kit. Muscles were homogenized in ice-cold distilled water and the protein concentrations were determined using a DC protein concentration assay (BioRad). Samples were then diluted to $2.5 \mu \mathrm{g} / \mu \mathrm{L}$ using the reagents supplied in the kit. The fluorescent substrate, in conjunction with $100 \mu \mathrm{M}$ of the cosubstrate $\mathrm{NAD}^{+}$, was then incubated with $15 \mu \mathrm{L}$ of each sample for 45 minutes at room temperature (RT) in a $1 / 2$ volume 96-well white microplate. Once complete, $2 \mathrm{mM}$ Nicotinamide (a Sirt1 inhibitor) and the provided fluorescent developer were added to each well to halt the reaction and produce a fluorophore which is linearly related to Sirt1 activity. The intensity of the fluorescent signal was detected with an excitation wavelength of $360 \mathrm{~nm}$ and an emission wavelength of $460 \mathrm{~nm}$. Data is presented as fluorescent units normalized to the respective milligrams of protein used in each homogenate.

Ex vitro muscle physiological analyses. Isometric muscle contractile properties were examined in the plantaris muscles from the left leg of all experimental animals. The muscles were placed in an oxygenated Ringer's solution $(137 \mathrm{mM} \mathrm{NaCl}, 4.7 \mathrm{mM} \mathrm{KCl}, 3.4 \mathrm{mM} \mathrm{CaCl}$, 1.2 $\mathrm{mM} \mathrm{MgSO}_{4}, 1 \mathrm{NaH}_{2} \mathrm{PO}_{4}$, and $112 \mathrm{D}$-glucose), which was maintained at $20^{\circ} \mathrm{C}$. The Ringer's solution was aerated with $95 \% \mathrm{O}_{2}$ and $5 \% \mathrm{CO}(\mathrm{pH} 7.4)$. The distal end of the muscle was attached to a stationary plexiglass plate, and the proximal end fixed to the lever arm of a 300C dynamometer (Aurora Scientific, Aurora Ontario, Canada). The muscles were stimulated by passing a constant current through platinum plates positioned on each side of the muscle. Ex vitro isometric twitch and tetanic contractions were obtained using a Constant Current/Constant Voltage Stimulator (Aurora Scientific) that provided DC-square wave signals at a stimulation current of 12 Volts, with a $200 \mu$ s pulse width. Muscles were adjusted to the optimal muscle length $\left(L_{0}\right)$ by a micromanipulator that controls the base position of the electrode clamp. $L_{\circ}$ was established as the muscle length that produced maximal isometric twitch tension and was 
periodically checked by the same procedure throughout each experiment to maintain this length. Force-frequency isometric force records were obtained by stimulating the muscle at 10, 20, 40, 50,75 and $100 \mathrm{~Hz}$, with 3 minutes of rest between each contraction. Physiological contractile measures included peak isometric twitch force (PT), time to peak twitch contraction tension $(C T)$, and the $1 / 2$ relaxation time of twitch contraction (1/2RT), as previously described (7). Following isometric contractions, the muscles remained in the oxygenated Ringer's solution for 5 minutes prior to the repeated stimulation fatigue protocol. Muscle fatigue was assessed by stimulating the muscle at $40 \mathrm{~Hz}$ for 3 minutes utilizing a duty cycle of $330 \mathrm{~ms}$ of stimulation followed by $660 \mathrm{~ms}$ of rest (53). The fatigue index was calculated as the force from the last contraction expressed as a percentage of the force obtained on the first contraction. The contractile and fatigue measurements were analyzed off line using commercial software (DMI, Aurora Scientific).

Fiber characteristics. Muscle fiber type and cross-sectional area (CSA) analyses were performed in the plantaris muscles. Frozen tissue cross sections measuring $10-\mu m$ thick were cut from the mid-belly of the muscles, mounted on charged microscope slides (Fisher Scientific; Pittsburgh, PA), and stored at $-80^{\circ} \mathrm{C}$. For analysis, the slides were air-dried 30 minutes, washed in PBS, and incubated for 30 minutes at RT with $4 \%$ bovine serum albumin (BSA) in PBS. The tissue sections were incubated overnight at $4^{\circ} \mathrm{C}$ with antibodies directed against laminin (MAB1914; Millipore; Temecula, CA) to visualize the basal lamina of each muscle fiber, as well as one of the following individual myosin heavy chain (MyHC) antibodies: BA-D5 (for MyHC I; Developmental Studies Hybridoma Bank; lowa City, IA), SC-71 (for MyHC IIA; DSHB), and BFF3 (for MyHC IIB; DSHB) in order to determine both the whole muscle composition and mean cross-sectional area (CSA) for each respective fiber type. Next day, the slides were washed in PBS and incubated 1 hour at $37^{\circ} \mathrm{C}$ with secondary antibodies of donkey anti-rat rhodamine (712-025-150; Jackson ImmunoResearch Laboratories; West Grove, PA) to visualize the basal 
lamina, and Alexa Fluor 488 (A10680; Invitrogen; Eugene, OR) to visualize the MyHC fibers. The sections were mounted with 4', 6-diamidino-2-phenylindole (DAPI) (Vectashield Mounting Medium; Vector Laboratories; Burlingame, CA) to visualize the nuclei. Four images from nonoverlapping regions of each tissue cross-section stained for individual MyHC fibers were used for both fiber type proportions and respective CSA's. All images were taken with a Zeiss LSM 510 Meta confocal microscope (Carl Zeiss Microimaging Inc.; Thornwood, NY) at a magnification of 20X. Mean fiber CSA of respective fiber types was determined by planimetry and calculated by the ImageJ software (NIH; Frederick, MD). Fiber types are expressed as a proportion of MyHC-positive fibers to total fibers for each individual MyHC examined. The percentage of type IIX MyHC fibers was calculated as the remainder once the type I, IIA, and IIB MyHC fiber percentages had been subtracted from one-hundred percent.

Western blotting. Western blots were used to determine the relative amount of proteins in apoptotic signaling pathways following HS and R. Approximately seventy-five micrograms of muscle was homogenized in ice-cold modified RIPA buffer (50mM Tris, 1\% NP-40, 150mM $\mathrm{NaCl}, 1 \mathrm{mM}$ EDTA, $0.1 \%$ SDS, $0.5 \%$ Na-deoxycholate; $\mathrm{pH}:$ 7.4) containing protease and phosphatase inhibitors. The muscle homogenates were centrifuged at $1,000 \mathrm{~g}$ for 5 minutes at $4^{\circ} \mathrm{C}$, and the resulting supernatant was collected. The protein content of the samples was measured using the DC protein assay kit (BioRad; Hercules, CA). Thirty-five micrograms of protein was loaded into each well of a 4-12\% gradient polyacrylamide gel (Invitrogen; Carlsbad, CA) and separated by routine sodium dodecyl sulfate-polyacrylamide gel electrophoresis (SDSPAGE) for 2 hours at 135V. The proteins were then transferred to a nitrocellulose membrane for 2 hours at $35 \mathrm{~V}$. Non-specific protein binding was blocked by incubating the membranes in $5 \%$ nonfat milk in Tris-buffered saline containing $0.05 \%$ Tween 20 (TBST) at RT for 1 hour. Membranes were incubated overnight at $4^{\circ} \mathrm{C}$ on a rocking table with primary antibodies $(1: 1,000)$ directed against Bcl-xL (\#2762; Cell Signaling Technology; Boston, MA) and Bax 
(\#2772; Cell Signaling). Furthermore, a primary antibody $(1: 2,000)$ was directed against GAPDH (ab8245; Abcam; Cambridge, MA) for use as a loading control. The following day, the membranes were washed in TBST and incubated in appropriate dilutions of secondary antibodies (diluted in 5\% non-fat milk) conjugated to horseradish peroxidase for one hour at RT on a rocking table. The signals were developed using a chemiluminescent substrate (Lumigen; Southfield, MI) and visualized by exposing the membranes to $x$-ray film (BioMax MS-1; Eastman Kodak, Rochester, NY). Digital records of the bands were captured using a Kodak 290 camera and quantified using one-dimensional analysis software (Eastman Kodak) as optical density $\times$ band area, expressed in arbitrary units relative to appropriate loading controls.

Identification of apoptotic nuclei. Frozen tissue 10- $\mu \mathrm{m}$ thick cross sections were obtained from the plantaris muscles and mounted on charged microscope slides (Fisher Scientific; Pittsburgh, PA). Fluorescent labeling of terminal dUTP nick-end labeling (TUNEL) with lamina allowed the detection of apoptotic nuclei in the muscle sections. This was accomplished using a slight modification to the method previously reported for our lab (219). Briefly, the tissue sections were air dried, fixed with $4 \%$ paraformaldehyde, and permeabilized with $0.2 \%$ Triton X100 in PBS for 30 minutes each at room temperature. The tissues were incubated overnight at $4^{\circ} \mathrm{C}$ in a rat anti-lamina monoclonal antibody (Millipore). Sections were incubated the following day with donkey anti-rat rhodamine conjugated secondary antibody (Jackson ImmunoResearch), along with the TUNEL reaction mixture (11-684-795-910; Roche Diagnostics; Indianapolis, IN) in a humidified chamber at $37^{\circ} \mathrm{C}$ for 1 hour in the dark. The exclusion of the TdT enzyme in the TUNEL reaction mixture on one of the tissue sections on each slide was included as a negative control. The sections were mounted with DAPI (Vectashield) in order to visualize nuclei and viewed under a Zeiss LSM 510 Meta confocal microscope (Carl Zeiss Microimaging Inc.; Thornwood, NY). The number of TUNEL and DAPIpositive nuclei that were immediately adjacent to, or beneath the basal lamina were counted. 
Data is expressed as an apoptotic index, calculated as the percentage of TUNEL-positive nuclei out of the total myonuclei (i.e., DAPI-positive nuclei) pool. The apoptotic index was determined from four non-overlapping regions of each tissue cross section visualized with a 20X objective.

BrdU detection. Frozen tissue cross sections measuring $10-\mu \mathrm{m}$ thick from the plantaris muscle were mounted on charged microscope slides (Fisher Scientific; Pittsburgh, PA). Briefly, tissue sections were air dried, washed in PBS, fixed in methanol:acetone $(1: 1)$ at $-20^{\circ} \mathrm{C}$ for 5 minutes, and permeabilized with $0.4 \%$ Triton X-100 in PBS. Sections were then denatured using Reagent 2 and blocked with Reagent 3, both components in the Calbiochem BrdU Immunohistochemistry System (HCS30; EMD Biosciences, Inc.; La Jolla, CA). Sections were incubated overnight in a biotinylated mouse anti-BrdU primary antibody. On the following day, sections were incubated with a secondary antibody of Fluorescein Avidin DCS (A-2011; Vector Laboratories, Inc.; Burlingame, CA) and subsequently blocked in normal goat serum. They were then incubated overnight in a rat anti-lamina monoclonal (Millipore) primary antibody. Sections were incubated the following day with a donkey anti-rat rhodamine conjugated (Jackson ImmunoResearch) secondary antibody in a humidified chamber at $37^{\circ} \mathrm{C}$ for 1 hour in the dark. Finally, the sections were mounted with DAPI-containing mounting medium (Vectashield) in order to visualize nuclei. Immunofluorescence was visualized with a Zeiss LSM 510 Meta confocal microscope (Carl Zeiss). Images were taken from four non-overlapping regions of each tissue section with a $20 \mathrm{X}$ objective.

Statistical analysis. All results are reported as means \pm SD. Differences in means between groups were determined by multiple analysis of variance (MANOVA) Hotelling's T-Square test. Bonferroni post hoc analyses were subsequently performed between significant means. A pvalue $<0.05$ was considered significant. 


\section{$\underline{\text { Results }}$}

Body weight. We measured the bodyweight of all animals at the beginning of the one-week pretreatment period (Start), immediately prior to suspension (Day 0), following suspension (HS), and following the recovery period $(R)$. There were no differences in bodyweight between any of the groups at the beginning of the experimental protocol. Furthermore, no significant changes in bodyweight were observed in the cage control animals at any of the time points. In the suspended animals, HS induced a significant loss of bodyweight in all of the animals as compared to the first day (Start) of the experiment, with decreases of $24.6 \%$ and $18.0 \%$ observed in the vehicle and resveratrol treated groups, respectively. These significant losses continued into the $\mathrm{R}$ period as well, with vehicle and resveratrol treated animals losing an additional $3.7 \%$ and $5.6 \%$ of body mass, respectively, between the end of $\mathrm{HS}$ and the end of the R period. However, resveratrol was unable to attenuate any of these bodyweight reductions, as there were no significant differences observed between the vehicle and resveratrol treated animals at any of the individual time points of the study (Figure $1 \mathrm{~A}$ ).

Muscle wet weight. Upon sacrifice, the plantaris muscle wet weights were examined to assess changes in muscle mass following the suspension and recovery periods. It was determined that HS induced significant decreases $(\mathrm{P}<.001)$ in absolute muscle mass in both the vehicle and resveratrol treated groups as compared to the HS control animals (Figure 1B). However, there were no differences observed between the vehicle and resveratrol treated animals. Following the $\mathrm{R}$ period, the wet weights from both the vehicle and resveratrol treated animals fully recovered to that of the control animals' muscle mass. Moreover, there were no significant differences observed between the vehicle and resveratrol treated groups. 
Muscle contractile characteristics. Freshly isolated plantaris muscles were attached to a force transducer in order to determine various muscle contractile properties following HS and R. Following $\mathrm{HS}$, there was a significant increase $(\mathrm{P}<.001)$ in $\mathrm{CT}$ during a twitch contraction in both the vehicle $(78.6 \pm 5.3 \mathrm{~ms})$ and resveratrol $(86.2 \pm 10.6 \mathrm{~ms})$ treated groups as compared to the cage controls (38.7 $\pm 6.8 \mathrm{~ms})$. However, resveratrol was unable to attenuate this increase (Figure $2 \mathrm{~A})$. In the $\mathrm{R}$ group, the CT of both the vehicle $(78.0 \pm 6.4 \mathrm{~ms})$ and resveratrol $(86.2 \pm$ $10.6 \mathrm{~ms})$ animals was significantly greater than that of the $\mathrm{R}$ Con $(62.0 \pm 8.0 \mathrm{~ms})$ animals (Figure 2B). There were no differences observed when comparing the CT of the vehicle and resveratrol treated animals.

Next, we examined the $1 / 2$ RT during a twitch contraction. In the HS group, the $1 / 2$ RT was significantly shorter in the vehicle treated $(47.4 \pm 11.8 \mathrm{~ms})$ animals compared to either the resveratrol $(65.8 \pm 7.8 \mathrm{~ms})$ or cage control $(69.8 \pm 9.6 \mathrm{~ms})$ groups (Figure $2 \mathrm{~A})$. Moreover, there were no differences between the resveratrol and cage control groups at this time point. This same trend also continued into the $\mathrm{R}$ period (Figure $2 \mathrm{~B}$ ). While there were no differences in the $1 / 2 \mathrm{RT}$ between the resveratrol $(71.3 \pm 18.1 \mathrm{~ms})$ and cage control $(61.2 \pm 13.0 \mathrm{~ms})$ groups, the $1 / 2$ RT of the vehicle treated $(42.7 \pm 3.8 \mathrm{~ms})$ group was significantly shorter than both of these two groups.

A fatigue index (see "Materials and Methods") was examined in order to determine if resveratrol had improved the muscles fatigue tolerance during a series of contractions. As expected, HS elicited a significant decrease in the muscles resistance to fatigue. Both the vehicle $(11.3 \pm 2.6 \%)$ and resveratrol $(11.6 \pm 2.9 \%)$ animals had significantly reduced fatigue indexes as compared to the cage control $(16.6 \pm 1.8 \%)$ group (Figure $2 \mathrm{C})$. However, there were no differences observed between the resveratrol and vehicle treated animals. In the $\mathrm{R}$ animals, the vehicle treated animals had a similar fatigue index $(10.9 \pm 1.6 \%)$ as the resveratrol $(9.5 \pm$ $1.8 \%$ ) fed group; however the fatigue index of the resveratrol group was significantly lower than the cage control $(14.8 \pm 4.8 \%)$ group after reloading (Figure $2 \mathrm{C}$ ). 
Lastly, we plotted the force-frequency curve for the HS and R groups. In the HS animals, a frequency of $20 \mathrm{~Hz}$ produced a significant rightward shift in both the vehicle and resveratrol treated groups (Figure 2D). At $50 \mathrm{~Hz}$, there remained a significant rightward shift in the resveratrol animals curve compared to both the cage control and vehicle treated animals. In contrast, there were no differences observed between the vehicle treated and cage control groups. At $75 \mathrm{~Hz}$, both the vehicle and resveratrol treated groups reached their peak relative forces, which shifted their curves significantly above that of the cage control group. Furthermore, the curve for the vehicle treated group was significantly higher than that for the resveratrol treated group.

In the R animals, both the vehicle and resveratrol treated groups' force-frequency curves were significantly shifted to the right of the cage control curve at 10,20 , and $50 \mathrm{~Hz}$ (Figure 2E). However, at none of these frequencies did the curve of either the vehicle or resveratrol groups differ from each other. While there remained no difference between the curves of the vehicle and resveratrol treated groups at $75 \mathrm{~Hz}$, only the curve for the resveratrol animals maintained a significant rightward shift from that of the cage controls.

Sirt1 activity. Blood plasma analysis of the resveratrol treated animals revealed a resveratrol concentration ranging from 176 to $5430 \mathrm{ng} / \mathrm{ml}$ in the HS group and from 81 to $934 \mathrm{ng} / \mathrm{ml}$ in the $\mathrm{R}$ group. There were no traces of resveratrol in the plasma of any of the vehicle treated animals. The relative activity of Sirt 1 in the plantaris muscles of all of the animals was assessed using a fluorometric assay. In the HS animals, there were no significant differences in Sirt1 activity between any of the groups, although there was a trend towards higher levels in the resveratrol treated animals (Figure 3). This trend continued with the $\mathrm{R}$ groups as well, although the differences between the groups was less than it was in the HS animals. This was due to both the $\mathrm{R}$ control and vehicle treated animals Sirt1 activity levels being slightly increased from that of their respective HS groups. 
MyHC profile. To determine if resveratrol had any effect on fiber composition following HS or $\mathrm{R}$, we examined the myosin heavy chain (MyHC) profile of the plantaris muscles. Following $\mathrm{HS}$, the relative percentage of type I MyHC fibers was significantly reduced in both of the groups subjected to suspension. However, there were no differences between the vehicle and resveratrol treated animals, although the mean percentage was slightly higher in the resveratrol group (Figure 4A). For the type IIA MyHC fibers, the resveratrol treated animals had a greater percentage than either the vehicle treated or cage control animals, which showed no differences between the two. Suspension significantly increased the percentage of type IIB fibers, although resveratrol had no effect on the composition of this fiber type compared to the vehicle treatment. In contrast, the percentage of type IIX MyHC fibers was significantly reduced after suspension in the resveratrol treated animals from that of the control muscles. However, there were no significant differences between the two suspended groups.

In the $R$ animals, the percentage of type I fibers remained significantly diminished in the animals that had previously been suspended. However, there was an increase in this fiber type in the vehicle treated animals to where the resveratrol treated animals no longer had a greater proportion of this fiber type (Figure 4B). While the percentage of type IIB fibers remained significantly elevated in both the vehicle and resveratrol treated animals, there remained no differences between these two groups. Lastly, although the resveratrol treated animals maintained a reduced percentage of type IIX MyHC fibers, the proportion of these fibers were also significantly reduced in the vehicle treated animals as well.

Fiber size. Muscle fiber CSA measurements were determined by planimetry and were analyzed for each type I, IIA, and IIB MyHC fibers. In the HS animals, the suspension induced significant muscle atrophy in all of the fiber types examined. In type I fibers, no differences in mean fiber CSA measurements were noted between the vehicle and resveratrol treated 
animals, although both had significantly smaller fibers than the cage control animals. In type IIA fibers, only the vehicle treated animals had myofibers with mean CSA's significantly smaller than that of the cage control animals (Figure 4C). The mean CSA's from the resveratrol treated animals were no different than the control animals, indicating that resveratrol partially preserved muscle fiber area during HS in this particular fiber type. In the type IIB MyHC fibers, both the vehicle and resveratrol treated groups mean CSA measurements were significantly smaller than those of the cage control animals. However, no significant differences were noted between the vehicle and resveratrol treated animal's myofibers in this fiber type following HS.

In the $\mathrm{R}$ animals, the same relative pattern persisted as it did in the HS animals (Figure 4D). In type I MyHC fibers, the mean CSA remained significantly smaller in both the vehicle and resveratrol treated animals as compared to the cage controls. In the type IIA fibers, only the vehicle treated animals had a significantly reduced mean CSA compared to the cage control animals. While the mean CSA of the resveratrol treated animal's fibers were not statistically different than the vehicle treated animals, the reduction in myofiber size from control levels appeared to be partially attenuated with resveratrol treatment, as these differences did not reach significance. Moreover, although the mean type IIB fiber CSA remained significantly reduced in the vehicle treated animals, the mean CSA of the resveratrol treated animals somewhat recovered and was no longer statistically smaller than the control fibers.

BrdU incorporation. A BrdU pellet was implanted into all of the $\mathrm{R}$ animals. As we were most interested in the regenerative period following $\mathrm{HS}$, these animals received the pellet immediately upon being released from suspension. BrdU-positive myonuclei were expressed per 100 myofibers. BrdU incorporation was very limited in the cage control $(0.34 \pm 0.61)$ animals, as there was no stimulus to activate any available satellite cells from quiescence under these homeostatic conditions (Figure 5). With the significant atrophy we observed in all of the animals subjected to suspension, it was not surprising that there was a significant increase in 
BrdU incorporation in both the vehicle $(7.80 \pm 3.23)$ and resveratrol $(11.00 \pm 3.54)$ treated animals, as satellite cells were activated upon reloading, presumably in an attempt to recover the muscle mass lost during HS. However, there were no statistical differences observed in the incorporation of BrdU between the vehicle and resveratrol groups.

Apoptotic signaling. We have previously characterized apoptotic protein changes during hindlimb suspension and reloading (36). In this study, western blots were used to determine the relative levels of the anti-apoptotic $\mathrm{Bcl}-\mathrm{xL}$ and pro-apoptotic Bax proteins as representative proteins in the apoptotic signaling pathway. Protein levels were normalized to GAPDH protein levels and are expressed in arbitrary units. In the HS group, there were no differences observed between any groups in Bax protein content (Figure 6A). However, Bcl-xL protein levels were significantly increased in the resveratrol $(0.49 \pm 0.12)$ treated group following HS, while no changes were observed in the vehicle $(0.20 \pm 0.16)$ treated group as compared to the cage control $(0.28 \pm 0.11)$ animals (Figure $6 \mathrm{~B})$.

In the $\mathrm{R}$ group, there were once again no differences observed in Bax protein content between the vehicle $(0.80 \pm 0.34)$ and resveratrol $(0.68 \pm 0.35)$ treated animals, nor were these values different than those of the cage control $(0.84 \pm 0.32)$ animals (Figure $6 \mathrm{~A})$. While the Bcl$x \mathrm{~L}$ protein levels remained low in the cage control $(0.25 \pm 0.16)$ animals, these values increased significantly in both the vehicle $(0.61 \pm 0.15)$ and resveratrol $(0.65 \pm 0.14)$ treated animals, although there were no differences observed between these two groups (Figure 6B).

To complement the western blot analysis, we used a TUNEL assay in order to determine the relative amount of myonuclei undergoing apoptosis immediately following HS (Figure 6C, 6E) and $\mathrm{R}$ (Figure 6D, 6E). As expected (101), both HS groups experienced a significant increase in apoptosis following HS. While insignificant, the mean increase in the apoptotic index was $29 \%$ higher in the vehicle $(1.35 \pm 0.49)$ treated animals compared to the resveratrol 
$(0.96 \pm 0.50)$ treated animals. In the R group, the TUNEL index remained significantly elevated in both the vehicle $(1.90 \pm 0.50)$ and resveratrol $(1.42 \pm 0.90)$ treated animals. 


\section{Discussion}

In this study we examined only aged animals, because we were interested in the effects of resveratrol on muscle atrophy and recovery following disuse in aged animals, and not in identifying differences in response between young and old animals. As expected $(101 ; 117$; 256), a fourteen day period of suspension induced significant reductions in both body and muscle weights in the aged animals. Although both the vehicle and resveratrol treated animals lost this body weight during the suspension period, resveratrol had no effect on the overall body mass changes. This is in agreement with several other studies in which resveratrol had no effect on fluctuations in body mass following suspension $(95 ; 123 ; 172)$. Furthermore, this trend continued into the recovery period. While the body masses of both the vehicle and resveratrol treated animals maintained a slight decline in body mass through this period, resveratrol did not have any measurable effect.

Following HS, the muscle wet weights of both the vehicle and resveratrol treated animals were significantly reduced. However, resveratrol was unable to attenuate these losses in muscle mass. Although a high dose $(400 \mathrm{mg} / \mathrm{kg})$ of resveratrol has been shown to protect soleus muscles from HS-induced atrophy (172), lower doses (12.5 mg/kg) have been shown to not have any effect on this atrophy in gastrocnemius muscles (123). Thus it is likely that any protective effects of resveratrol against disuse atrophy are muscle and/or dose specific. The latter appears to be the case for our current study, as in addition to the plantaris muscles, resveratrol was also unable to attenuate the HS-induced decreases in muscle mass in the soleus or gastrocnemius muscles (data not shown) of these animals. Moreover, there was no improvement in muscle mass with resveratrol supplementation following the $\mathrm{R}$ period. At this stage, both the vehicle and resveratrol animals which had been previously suspended had muscle weights that were not statistically different from that of the cage control animals, although the means of each were still slightly reduced. Thus, our observations lead us to 
speculate that our current dose of resveratrol was insufficient to maximize any potential benefits on muscle mass in this particular model. Furthermore, as the masses from both the vehicle and resveratrol animals were fully restored following fourteen days of recovery, it is not known whether resveratrol supplementation is able to shorten this recovery.

We examined several contractile properties of the muscles from both the HS and R animals to determine if resveratrol had any effect on muscle function. Upon examination of the muscle twitch properties, we observed a suspension-induced decrease in the $1 / 2 R T$, as was expected $(79 ; 172)$. However, this decrease was completely ablated in both the HS and R groups that received the resveratrol supplementation. This is in agreement with another recent study, which found resveratrol to be effective in preventing decreases in $1 / 2 R T$ following a period of disuse (172). This could occur by several means including maintenance of SERCA proteins and improved calcium uptake at the sarcoplasmic reticulum, but this was not assessed in the current study. We next examined the fatigue properties of the plantaris from animals in the HS and $R$ groups. The fatigue index revealed a decrease in the ability of the plantaris to withstand repeated contractions in both of the groups subjected to suspension. Although there were no differences in the fatigue index of the vehicle and resveratrol treated animals following $\mathrm{HS}$, it was determined that only the resveratrol treated animals had a fatigue index value which was significantly less than that of the control animals following the R period. While it was unexpected that the resveratrol treated animals would have a reduced fatigue index, the observation that resveratrol treatment does not improve upon the index is not without precedent. In other studies involving resveratrol supplementation $(80 ; 122)$, no improvements were observed in a fatigue index protocol. However, it must also be noted that in one study in which young, dystrophic mdx mice were supplemented with resveratrol, there was a significant improvement of the observed fatigue index (212). Given the contradicting results of these studies, it is only possible to state that the possible effects of resveratrol on skeletal muscle fatigue remain inconclusive, and very likely are conditional to the specific experimental models 
used to observe these effects. Therefore, further research in this area is needed to reconcile these discrepancies.

In examining the force-frequency curves of both the HS and R groups, we observed a rightward shift in both of the groups that had been subjected to suspension. This shift was prominent at the lower frequencies in the HS groups and was further exacerbated in the R vehicle and resveratrol treated groups. This rightward shift is suggestive of a faster muscle phenotype, which is consistent with our observations of the fiber type composition of these muscles. While the proportion of type I MyHC fibers decreased in both the resveratrol and vehicle treated animals following suspension, there was a very significant increase in type IIB fibers. Concomitantly, there was also a slight increase in type IIA MyHC fibers, although this only reached significance in the resveratrol treated $\mathrm{HS}$ animals and did not continue into the $\mathrm{R}$ period. These results were slightly different than that reported in a recent study by Momken and colleagues (36). Under similar experimental conditions as our present study, they observed that resveratrol supplementation attenuated the decrease in type I MyHC fibers following suspension (172). They attributed this finding to the ability of resveratrol, acting primarily through Sirt1, to increase the expression of PGC-1 $1 \alpha$, which has been shown to promote the maintenance of slow-twitch fiber's oxidative characteristics (99). However, our current findings most likely differ from the Momken et al. findings due to the specific muscles examined in each study. While our current study utilized the primarily glycolytic plantaris muscles, Momken et al. (36) examined the much more oxidative soleus muscles. Nevertheless, the effects of resveratrol might be muscle specific because the type I fibers in the plantaris of resveratrol and vehicle treated animals had similar reductions in cross sectional area after HS or R. Thus, the protective effect on type I fiber composition observed reported by Momken and colleagues (36) did not appear to occur in our present study.

As is typical of muscles subjected to periods of unloading, we observed a decrease in the mean CSA of the plantaris muscles. Although resveratrol supplementation did not have an 
effect on type I fiber size following either HS or R, it did have an impact on type II fiber area. Following HS, resveratrol effectively attenuated the significant loss of type IIA fiber area. Furthermore, resveratrol benefitted the type II fibers following the R period, as both type IIA and IIB fiber areas were not different than control fibers, whereas the type II fibers of the vehicle treated animals remained significantly smaller than control muscle fibers during the $\mathrm{R}$ period. It was not surprising that resveratrol benefitted muscle fiber size, given that similar results have been observed in several recent studies $(3 ; 172)$. These beneficial effects observed in our current study are most likely due to resveratrol being able to attenuate muscle protein degradation during conditions which normally would induce atrophy (3), and not due to an increased growth response per se during the period of recovery following our suspensioninduced atrophy protocol. This is supported in part by our examination of BrdU-positive nuclei during this recovery period. Analyzing the BrdU-positive nuclei (i.e., satellite cells) allowed us to observe the activation of these muscle precursor cells throughout the R period. However, we did not observe any differences between the vehicle and resveratrol treated animals in this context, indicating that our current dose of resveratrol was insufficient at increasing the activation, and thereby muscle growth potential, of the latent satellite cells. Thus, it is likely that the benefits of resveratrol in providing an increased muscle size recovery are due to its' attenuating the suspension-induced atrophy throughout the HS period.

Recent work has shown resveratrol to have beneficial effects on apoptosis in multiple different tissues $(4 ; 94 ; 123 ; 261)$. Therefore, we examined representative indicators of positive and negative apoptotic signaling to determine if resveratrol was beneficial following $\mathrm{HS}$ and $\mathrm{R}$ in our specific model. Although no changes were observed in Bax protein expression following either HS or $\mathrm{R}, \mathrm{Bcl}-\mathrm{xL}$ protein expression was significantly elevated in the resveratrol treated animals following HS. This is consistent with an attenuation of muscle fiber size during HS in resveratrol treated animals. However, there were no differences in the abundance of these apoptotic proteins observed between the vehicle and resveratrol treated animals following $\mathrm{R}$. 
Likewise, in examining TUNEL-positive nuclei, we did not observe any significant differences between the vehicle and resveratrol treated animals following either HS or R, although the mean TUNEL index values were slightly lower in the resveratrol treated animals. Thus it is likely that the beneficial, albeit minor, effects of resveratrol on $\mathrm{Bcl}-\mathrm{xL}$ protein levels were sufficient to partially mediate decrements in myofiber size which were observed following the HS period in our current study.

The findings from our present study provide evidence for the modest benefits of resveratrol supplementation in improving skeletal muscle regeneration following a period of disuse with a subsequent recovery. Although resveratrol did not preserve either body or muscle weight decrements following HS, it was able to induce favorable changes to type IIA and IIB muscle fiber CSA measurements and apoptotic signaling. This is particularly relevant in the elderly population which experience preferential atrophy of type II fibers in sarcopenic conditions. The potential caveat of this study may be that the dose of resveratrol used was simply not enough to elicit the full benefits of its regenerative capabilities on all fiber types. Thus, the evidence from this study warrants the further research into this subject, as resveratrol supplementation holds promise of a novel treatment to combat the decline of muscle function in the elderly following periods of disuse. 


\section{Figure Legends}

Figure 1. Body and muscle wet weights. A) Bodyweights measurements of all animals were taken immediately prior to the start of the one-week pretreatment period (Start), immediately prior to suspension (0), following the suspension period (HS), and again following the reloading period $(\mathrm{R})$. B) Plantaris muscle wet weights were analyzed either following HS or $\mathrm{R}$. \# $\mathrm{P}<.05$ vs. CC.

Figure 2. Plantaris muscle force characteristics. $A, B)$ Muscle twitch properties including CT and $1 / 2$ RT were analyzed in the plantaris muscles of both the HS (A) and R (B) groups. $C$ ) A fatigue index was calculated from a series of 128 consecutive contractions. $D, E)$ A forcefrequency curve was plotted for both the HS (D) and $\mathrm{R}(E)$ groups. ${ }^{*} \mathrm{P}<.05$ vehicle vs. resveratrol, \# $\mathrm{P}<.05$ vs. CC.

Figure 3. Sirt1 activity. The relative activity of Sirt1 was determined in all of the animals. Values are in fluorescent units normalized to the respective milligrams of protein used in each sample.

Figure 4. Muscle fiber composition and morphology. $A, B$ ) Individual MyHC fiber composition was analyzed in both the HS $(A)$ and $\mathrm{R}(B)$ groups to determine the relative composition of the plantaris muscles. $C, D$ ) Mean fiber area measurements were performed in type I, IIA, and IIB MyHC fibers in the HS (C) and R (D) groups. E) Representative images of fiber type staining (type I MyHC) for fiber type composition and CSA analyses for both the HS and $\mathrm{R}$ groups. Green fibers are MyHC-positive. Scale bars represent $50 \mu \mathrm{m}$. ${ }^{*} \mathrm{P}<.05$ vehicle vs. resveratrol, \# $\mathrm{P}<.05$ vs. CC. 
Figure 5. BrdU incorporation. In order to analyze cellular proliferation during the reloading period, a BrdU pellet was inserted into the $\mathrm{R}$ animals as they were released from suspension. $\mathrm{BrdU}$ incorporation is expressed as the number of BrdU-positive myonuclei per one-hundred fibers. \# P<.05 vs. CC.

Figure 6. Apoptosis. $A, B$ ) Western blots were utilized to determine the relative levels of proapoptotic $\operatorname{Bax}(A)$ and anti-apoptotic $B c l-x L(B)$ proteins. Units are expressed in arbitrary units relative to appropriate GAPDH protein levels. $C, D, E)$ DNA fragmentation was assessed with TUNEL staining in both the HS $(C, E)$ and $\mathrm{R}(D, E)$ groups. Figures $C$ and $D$ are representative images taken from the HS and $\mathrm{R}$ groups, respectively. ${ }^{*} \mathrm{P}<.05$ vs. vehicle, \# $\mathrm{P}<.05$ vs. CC. 
1. Alamdari N, Aversa Z, Castillero E, Gurav A, Petkova V, Tizio S and Hasselgren PO. Resveratrol prevents dexamethasone-induced expression of the muscle atrophy-related ubiquitin ligases atrogin-1 and MuRF1 in cultured myotubes through a SIRT1-dependent mechanism. Biochem Biophys Res Commun 417: 528-533, 2012.

2. Alcendor RR, Gao S, Zhai P, Zablocki D, Holle E, Yu X, Tian B, Wagner T, Vatner SF and Sadoshima J. Sirt1 regulates aging and resistance to oxidative stress in the heart. Circ Res 100: 1512-1521, 2007.

3. Alway SE. Attenuation of $\mathrm{Ca}(2+)$-activated ATPase and shortening velocity in hypertrophied fast twitch skeletal muscle from aged Japanese quail. Exp Gerontol 37: 665-678, 2002.

4. Baliga MS, Meleth S and Katiyar SK. Growth inhibitory and antimetastatic effect of green tea polyphenols on metastasis-specific mouse mammary carcinoma 4T1 cells in vitro and in vivo systems. Clin Cancer Res 11: 1918-1927, 2005.

5. Barani AE, Durieux AC, Sabido $\mathbf{O}$ and Freyssenet $\mathbf{D}$. Age-related changes in the mitotic and metabolic characteristics of muscle-derived cells. J Appl Physiol 95: 2089-2098, 2003.

6. Baumgartner RN, Koehler KM, Gallagher D, Romero L, Heymsfield SB, Ross RR, Garry PJ and Lindeman RD. Epidemiology of sarcopenia among the elderly in New Mexico. Am J Epidemiol 147: 755-763, 1998. 
7. Beauchamp JR, Heslop L, Yu DS, Tajbakhsh S, Kelly RG, Wernig A, Buckingham ME, Partridge TA and Zammit PS. Expression of CD34 and Myf5 defines the majority of quiescent adult skeletal muscle satellite cells. J Cell Biol 151: 1221-1234, 2000.

8. Boldrin L and Morgan JE. Activating muscle stem cells: therapeutic potential in muscle diseases. Curr Opin Neurol 20: 577-582, 2007.

9. Bortoli S, Renault V, Eveno E, Auffray C, Butler-Browne G and Pietu G. Gene expression profiling of human satellite cells during muscular aging using cDNA arrays. Gene 321: 145-154, 2003.

10. Burton LA and Sumukadas D. Optimal management of sarcopenia. Clin Interv Aging 5: 217-228, 2010.

11. Carlson ME, Hsu M and Conboy IM. Imbalance between pSmad3 and Notch induces CDK inhibitors in old muscle stem cells. Nature 454: 528-532, 2008.

12. Castillo EM, Goodman-Gruen D, Kritz-Silverstein D, Morton DJ, Wingard DL and BarrettConnor E. Sarcopenia in elderly men and women: the Rancho Bernardo study. Am J Prev Med 25: 226-231, 2003.

13. Chakravarthy MV, Davis BS and Booth FW. IGF-I restores satellite cell proliferative potential in immobilized old skeletal muscle. J Appl Physiol 89: 1365-1379, 2000. 
14. Charge SB, Brack AS and Hughes SM. Aging-related satellite cell differentiation defect occurs prematurely after Ski-induced muscle hypertrophy. Am J Physiol Cell Physiol 283: C1228-C1241, 2002.

15. Chen KD and Alway SE. Clenbuterol reduces soleus muscle fatigue during disuse in aged rats. Muscle Nerve 24: 211-222, 2001.

16. Collins CA, Olsen I, Zammit PS, Heslop L, Petrie A, Partridge TA and Morgan JE. Stem cell function, self-renewal, and behavioral heterogeneity of cells from the adult muscle satellite cell niche. Cell 122: 289-301, 2005.

17. Collins CA, Zammit PS, Ruiz AP, Morgan JE and Partridge TA. A population of myogenic stem cells that survives skeletal muscle aging. Stem Cells 25: 885-894, 2007.

18. Conboy IM, Conboy MJ, Smythe GM and Rando TA. Notch-mediated restoration of regenerative potential to aged muscle. Science 302: 1575-1577, 2003.

19. Cruz-Jentoft AJ, Baeyens JP, Bauer JM, Boirie Y, Cederholm T, Landi F, Martin FC, Michel JP, Rolland Y, Schneider SM, Topinkova E, Vandewoude M and Zamboni M. Sarcopenia: European consensus on definition and diagnosis: Report of the European Working Group on Sarcopenia in Older People. Age Ageing 39: 412-423, 2010.

20. de la Lastra CA and Villegas I. Resveratrol as an anti-inflammatory and anti-aging agent: mechanisms and clinical implications. Mol Nutr Food Res 49: 405-430, 2005. 
21. Dodd SL and Koesterer TJ. Clenbuterol attenuates muscle atrophy and dysfunction in hindlimbsuspended rats. Aviat Space Environ Med 73: 635-639, 2002.

22. Dolinsky VW, Jones KE, Sidhu RS, Haykowsky M, Czubryt MP, Gordon T and Dyck JR. Improvements in skeletal muscle strength and cardiac function induced by resveratrol during exercise training contribute to enhanced exercise performance in rats. J Physiol 590: 2783-2799, 2012.

23. Gu J, Song ZP, Gui DM, Hu W, Chen YG and Zhang DD. Resveratrol Attenuates DoxorubicinInduced Cardiomyocyte Apoptosis in Lymphoma Nude Mice by Heme Oxygenase-1 Induction. Cardiovasc Toxicol 2012.

24. Habold C, Momken I, Ouadi A, Bekaert V and Brasse D. Effect of prior treatment with resveratrol on density and structure of rat long bones under tail-suspension. J Bone Miner Metab 29: 15-22, 2011.

25. Handschin C, Chin S, Li P, Liu F, Maratos-Flier E, Lebrasseur NK, Yan Z and Spiegelman BM. Skeletal muscle fiber-type switching, exercise intolerance, and myopathy in PGC-1alpha musclespecific knock-out animals. J Biol Chem 282: 30014-30021, 2007.

26. Hao Y, Jackson JR, Wang Y, Edens N, Pereira SL and Alway SE. beta-Hydroxy-betamethylbutyrate reduces myonuclear apoptosis during recovery from hind limb suspensioninduced muscle fiber atrophy in aged rats. Am J Physiol Regul Integr Comp Physiol 301: R701R715, 2011. 
27. Harikumar KB and Aggarwal BB. Resveratrol: a multitargeted agent for age-associated chronic diseases. Cell Cycle 7: 1020-1035, 2008.

28. Hur YG, Suh CH, Kim S and Won J. Rosmarinic acid induces apoptosis of activated T cells from rheumatoid arthritis patients via mitochondrial pathway. J Clin Immunol 27: 36-45, 2007.

29. Hwee DT and Bodine SC. Age-related deficit in load-induced skeletal muscle growth. J Gerontol A Biol Sci Med Sci 64: 618-628, 2009.

30. Jackson JR, Ryan MJ and Alway SE. Long-term supplementation with resveratrol alleviates oxidative stress but does not attenuate sarcopenia in aged mice. J Gerontol A Biol Sci Med Sci 66: 751-764, 2011.

31. Jackson JR, Ryan MJ, Hao Y and Alway SE. Mediation of endogenous antioxidant enzymes and apoptotic signaling by resveratrol following muscle disuse in the gastrocnemius muscles of young and old rats. Am J Physiol Regul Integr Comp Physiol 299: R1572-R1581, 2010.

32. Karagounis LG and Hawley JA. Skeletal muscle: increasing the size of the locomotor cell. Int J Biochem Cell Biol 42: 1376-1379, 2010.

33. Lagouge M, Argmann C, Gerhart-Hines Z, Meziane H, Lerin C, Daussin F, Messadeq N, Milne J, Lambert P, Elliott P, Geny B, Laakso M, Puigserver P and Auwerx J. Resveratrol improves mitochondrial function and protects against metabolic disease by activating SIRT1 and PGC1alpha. Cell 127: 1109-1122, 2006. 
34. Magne H, Savary-Auzeloux I, Vazeille E, Claustre A, Attaix D, Anne L, Veronique SL, Philippe G, Dardevet $\mathbf{D}$ and Combaret $\mathbf{L}$. Lack of muscle recovery after immobilization in old rats does not result from a defect in normalization of the ubiquitin-proteasome and the caspase-dependent apoptotic pathways. J Physiol 589: 511-524, 2011.

35. MAURO A. Satellite cell of skeletal muscle fibers. J Biophys Biochem Cytol 9: 493-495, 1961.

36. Momken I, Stevens L, Bergouignan A, Desplanches D, Rudwill F, Chery I, Zahariev A, Zahn S, Stein TP, Sebedio JL, Pujos-Guillot E, Falempin M, Simon C, Coxam V, Andrianjafiniony T, Gauquelin-Koch G, Picquet F and Blanc S. Resveratrol prevents the wasting disorders of mechanical unloading by acting as a physical exercise mimetic in the rat. FASEB J 25: 3646-3660, 2011.

37. Pistilli EE, Siu PM and Alway SE. Interleukin-15 responses to aging and unloading-induced skeletal muscle atrophy. Am J Physiol Cell Physiol 292: C1298-C1304, 2007.

38. Rahman I, Biswas SK and Kirkham PA. Regulation of inflammation and redox signaling by dietary polyphenols. Biochem Pharmacol 72: 1439-1452, 2006.

39. Russell ST, Wyke SM and Tisdale MJ. Mechanism of induction of muscle protein degradation by angiotensin II. Cell Signal 18: 1087-1096, 2006.

40. Ryan MJ, Jackson JR, Hao Y, Williamson CL, Dabkowski ER, Hollander JM and Alway SE. Suppression of oxidative stress by resveratrol after isometric contractions in gastrocnemius muscles of aged mice. J Gerontol A Biol Sci Med Sci 65: 815-831, 2010. 
41. Seale P, Sabourin LA, Girgis-Gabardo A, Mansouri A, Gruss P and Rudnicki MA. Pax7 is required for the specification of myogenic satellite cells. Cell 102: 777-786, 2000.

42. Selsby JT, Morine KJ, Pendrak K, Barton ER and Sweeney HL. Rescue of dystrophic skeletal muscle by PGC-1alpha involves a fast to slow fiber type shift in the mdx mouse. PLoS One 7: e30063, 2012.

43. Shefer G, Van de Mark DP, Richardson JB and Yablonka-Reuveni Z. Satellite-cell pool size does matter: defining the myogenic potency of aging skeletal muscle. Dev Biol 294: 50-66, 2006.

44. Siu PM, Pistilli EE, Butler DC and Alway SE. Aging influences cellular and molecular responses of apoptosis to skeletal muscle unloading. Am J Physiol Cell Physiol 288: C338-C349, 2005.

\section{Suetta C, Hvid LG, Justesen L, Christensen U, Neergaard K, Simonsen L, Ortenblad N,} Magnusson SP, Kjaer M and Aagaard P. Effects of aging on human skeletal muscle after immobilization and retraining. J Appl Physiol 107: 1172-1180, 2009.

46. Waters DL, Baumgartner RN, Garry PJ and Vellas B. Advantages of dietary, exercise-related, and therapeutic interventions to prevent and treat sarcopenia in adult patients: an update. Clin Interv Aging 5: 259-270, 2010.

47. Wyke SM, Russell ST and Tisdale MJ. Induction of proteasome expression in skeletal muscle is attenuated by inhibitors of NF-kappaB activation. Br J Cancer 91: 1742-1750, 2004. 
48. Wyke SM and Tisdale MJ. Induction of protein degradation in skeletal muscle by a phorbol ester involves upregulation of the ubiquitin-proteasome proteolytic pathway. Life Sci 78: 2898-2910, 2006.

49. Xu J, Hwang JC, Lees HA, Wohlgemuth SE, Knutson MD, Judge AR, Dupont-Versteegden EE, Marzetti E and Leeuwenburgh C. Long-term perturbation of muscle iron homeostasis following hindlimb suspension in old rats is associated with high levels of oxidative stress and impaired recovery from atrophy. Exp Gerontol 47: 100-108, 2012.

50. Zhang J, Chen L, Zheng J, Zeng T, Li H, Xiao H, Deng X and Hu X. The protective effect of resveratrol on islet insulin secretion and morphology in mice on a high-fat diet. Diabetes Res Clin Pract 2012. 


\section{Fiqures}

\section{Figure 1A}

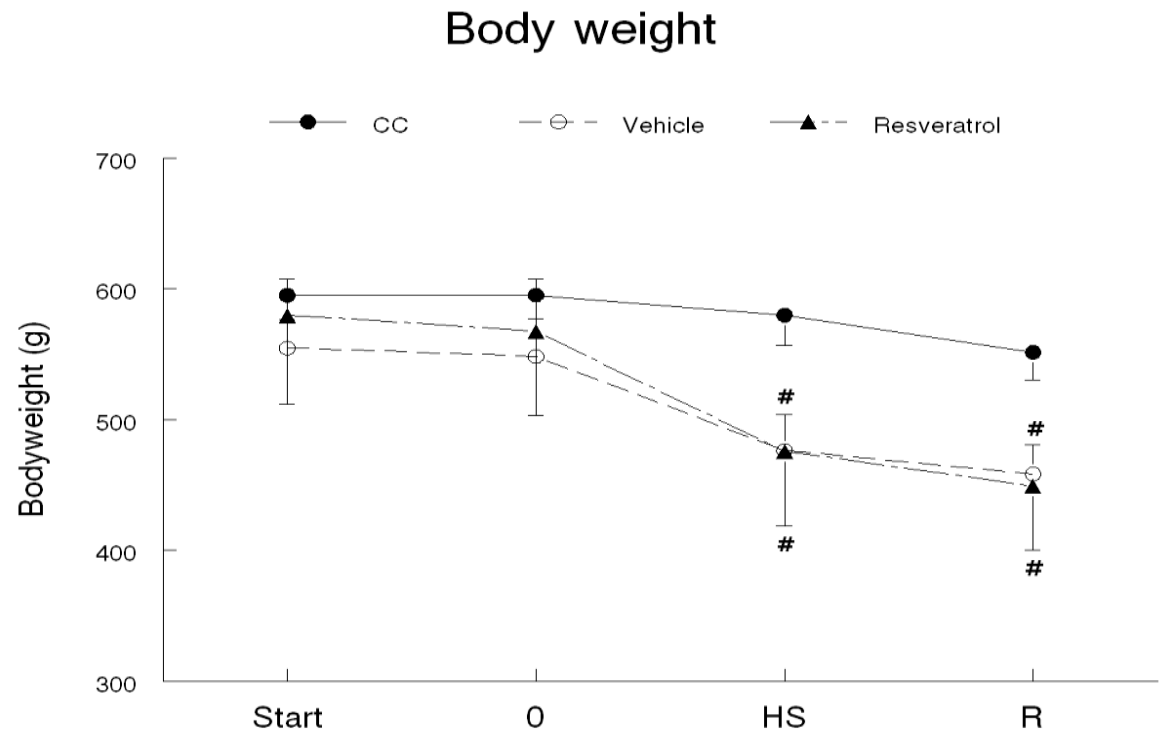

Figure 1B

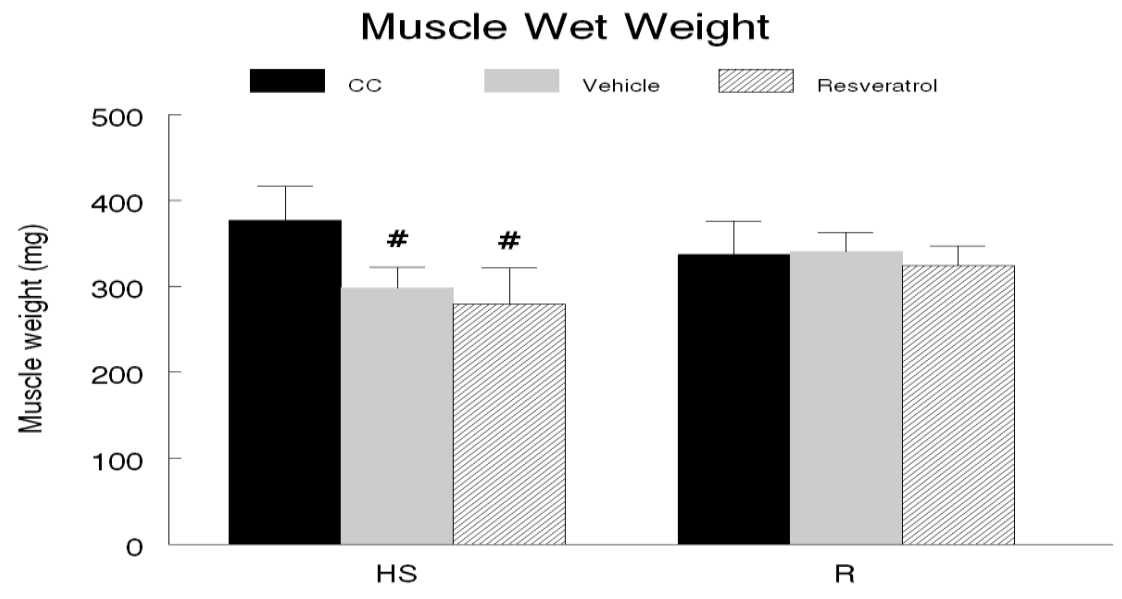


Figure 2A

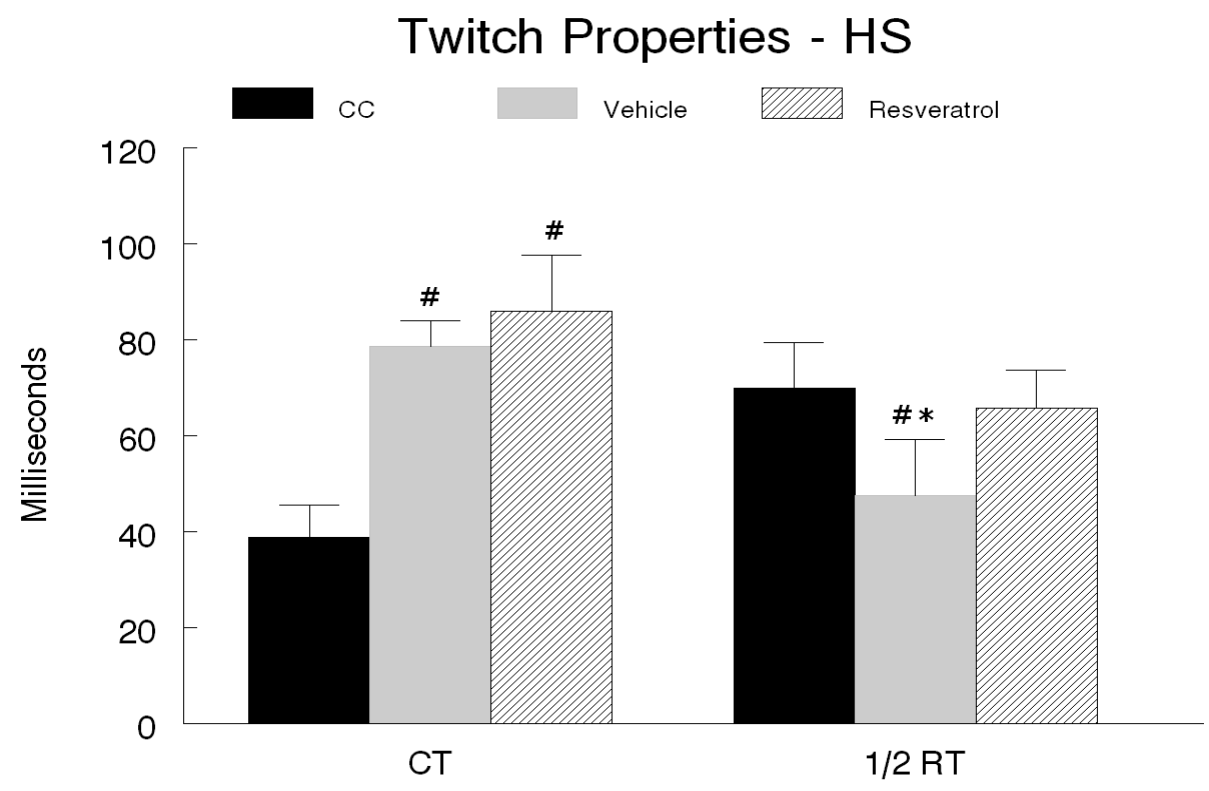

Figure 2B

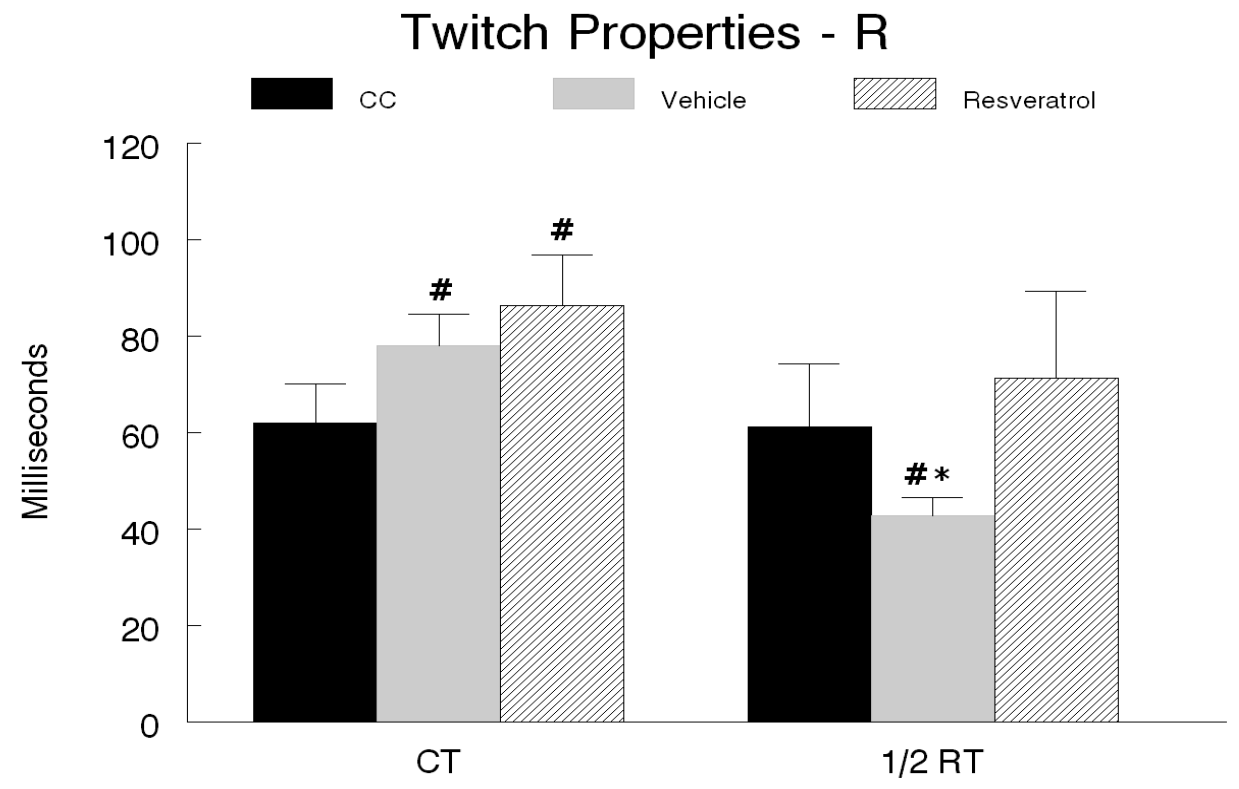


Figure 2C

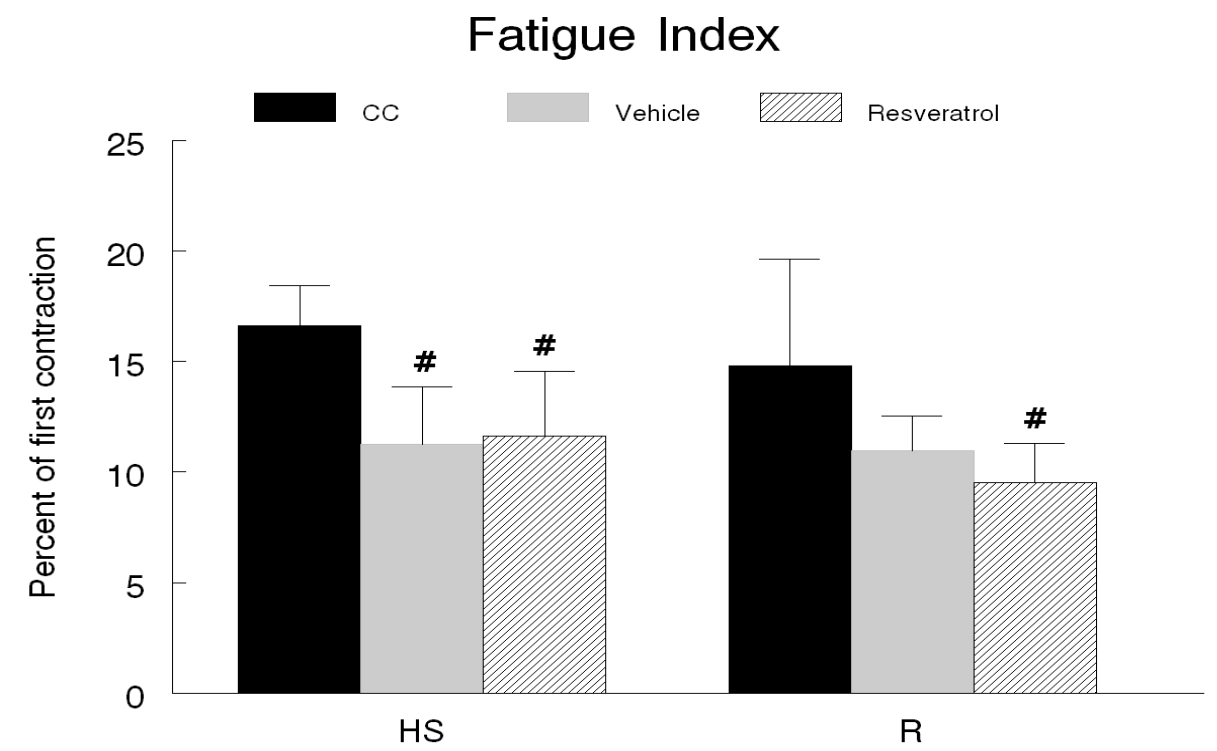

Figure 2D

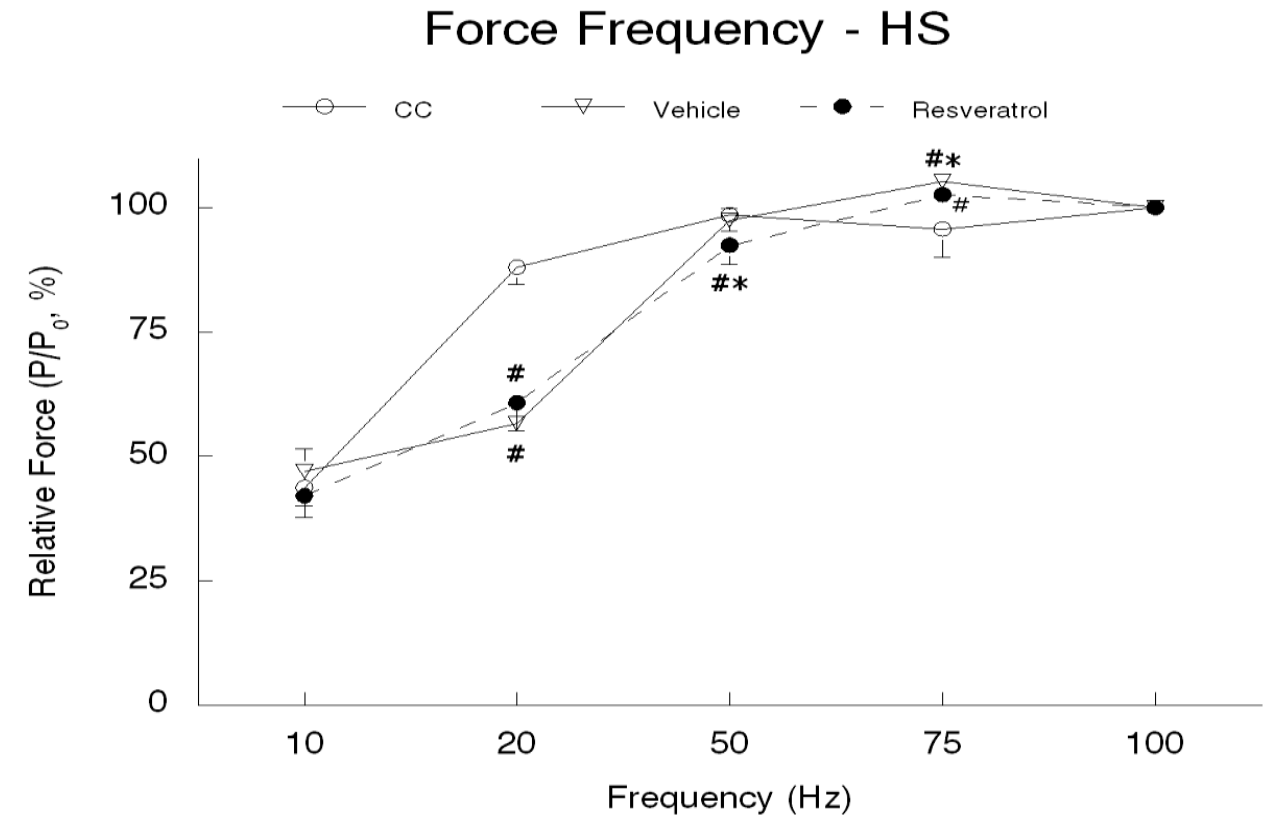


Figure 2E

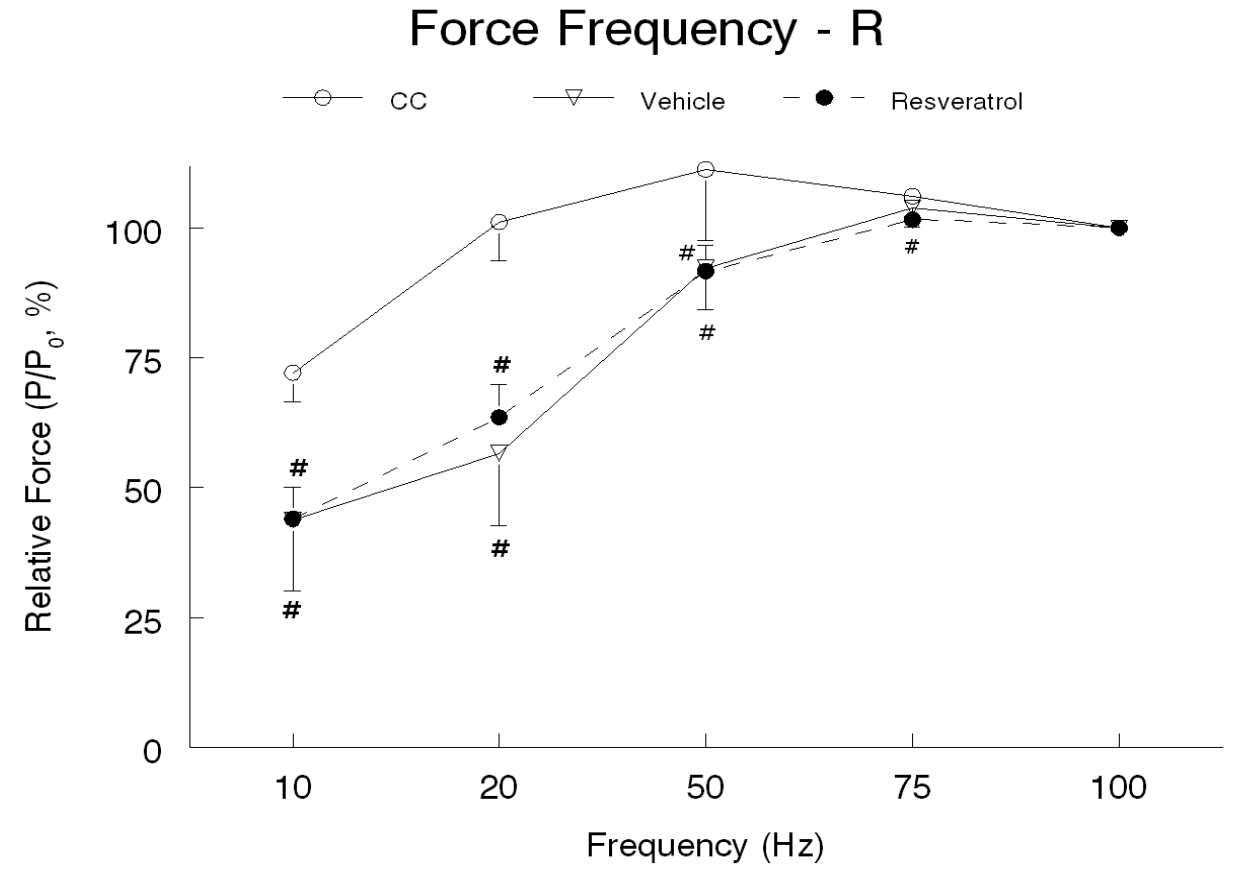

Figure 3

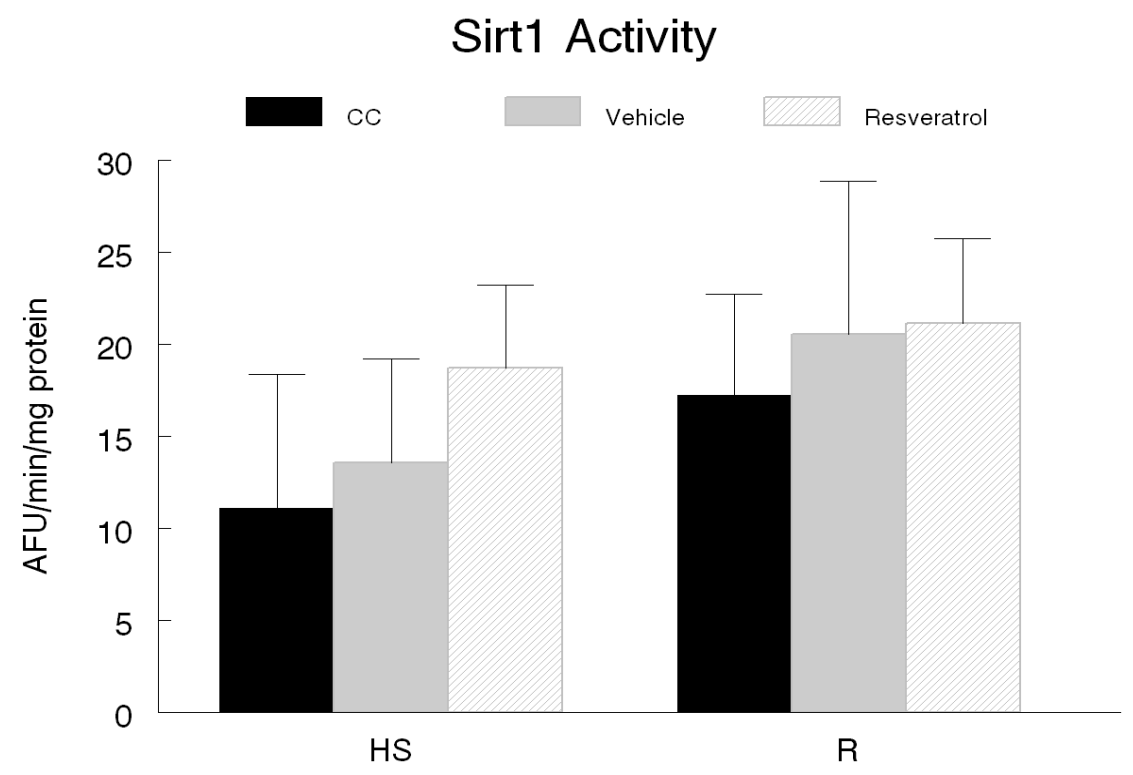


Figure 4A

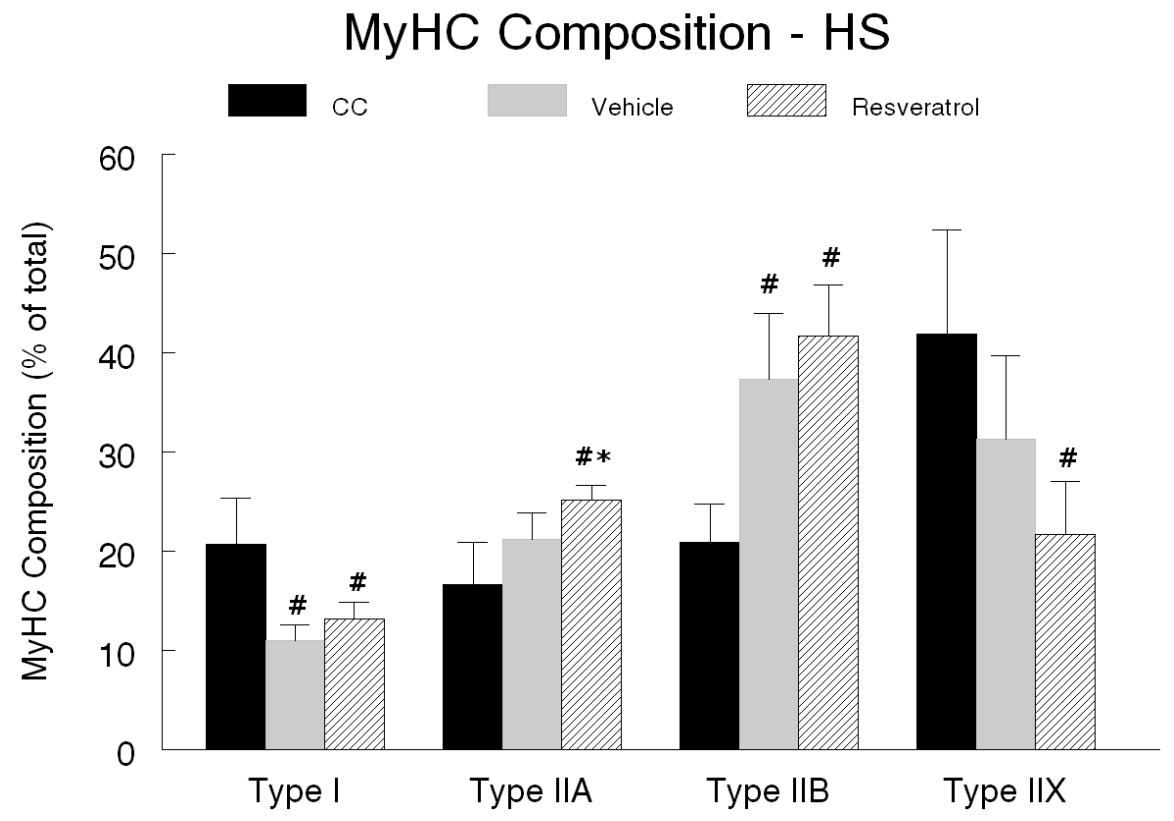

Figure 4B

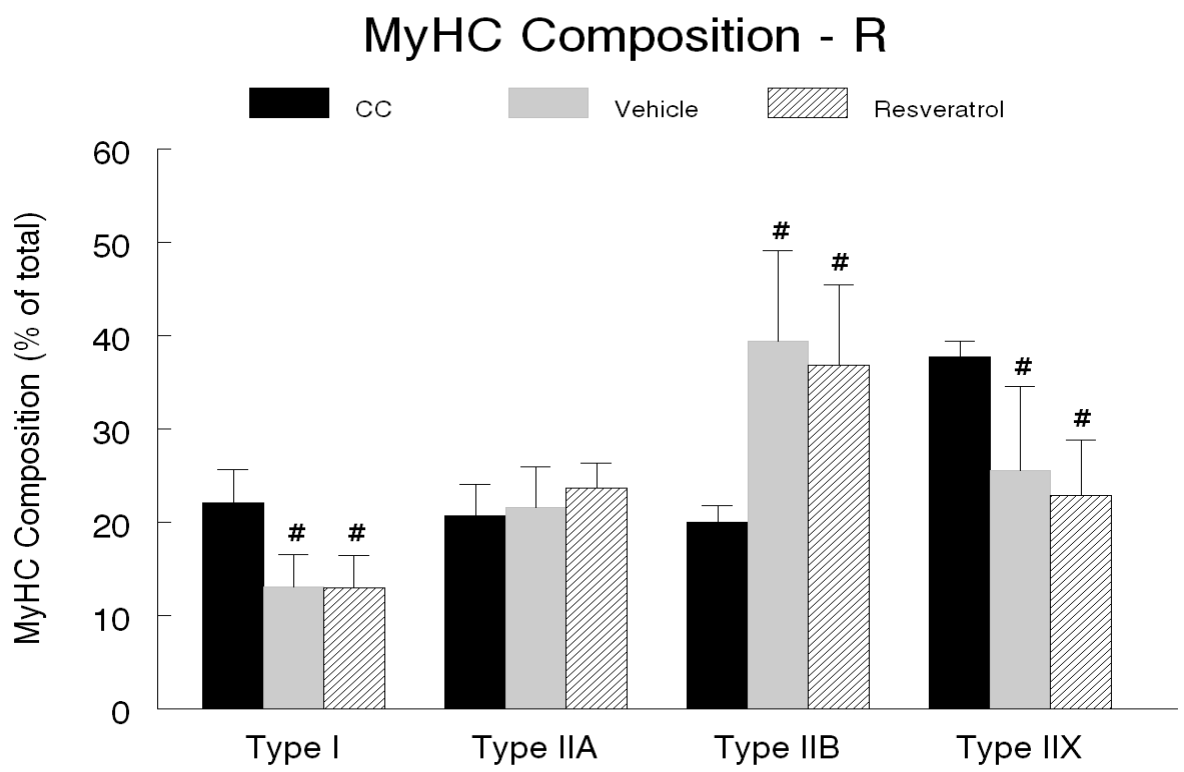


Figure 4C

Fiber CSA - HS

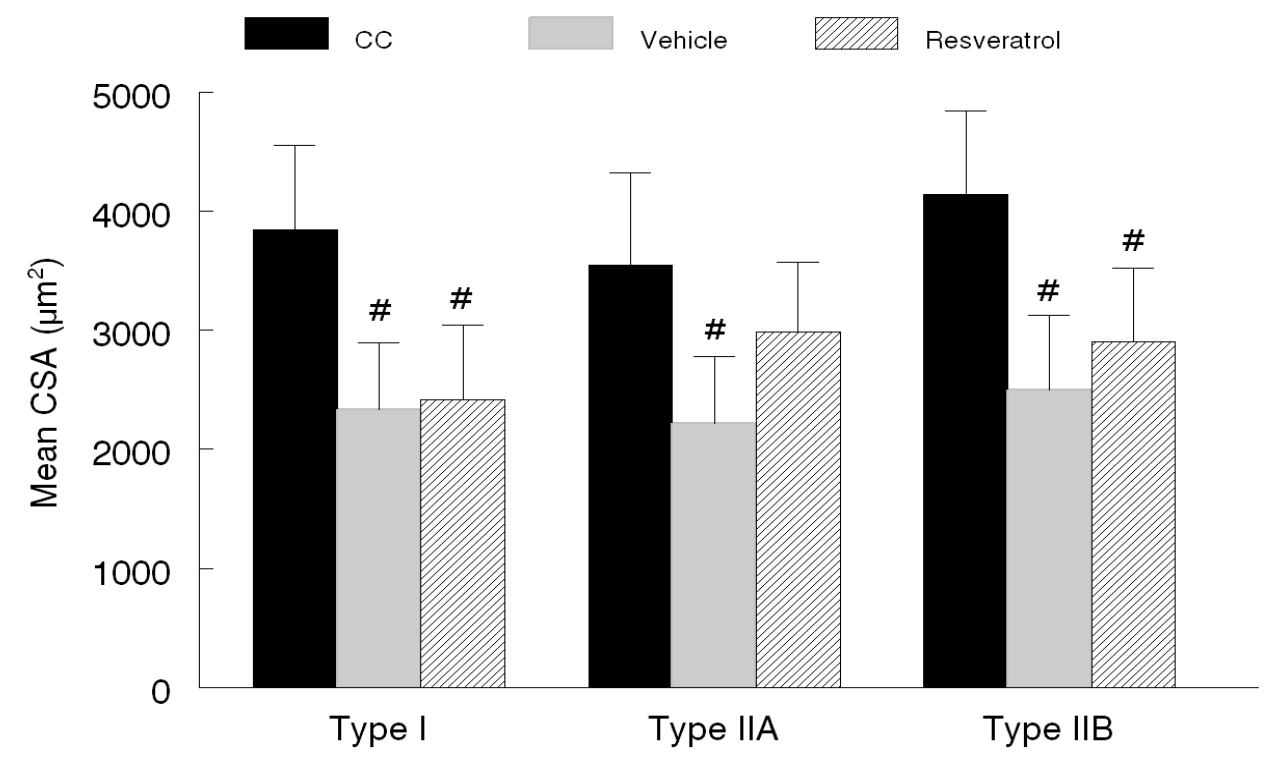

Figure 4D

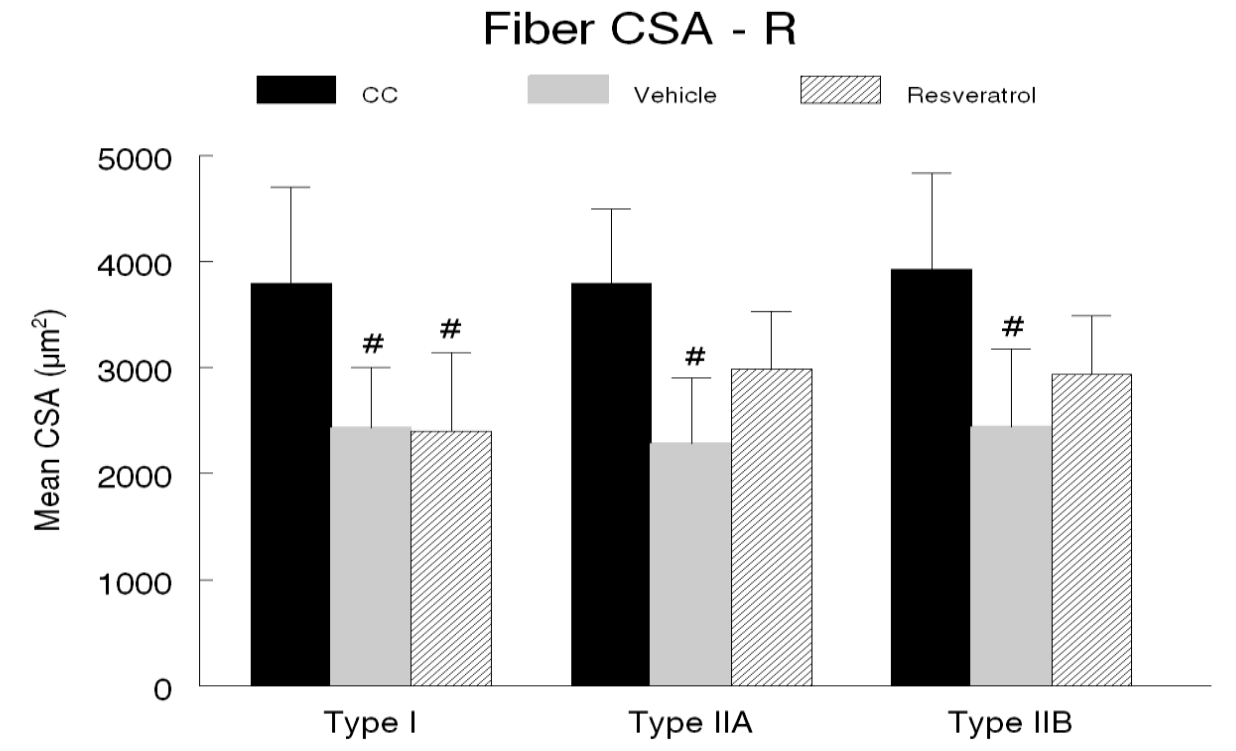


Figure 4E

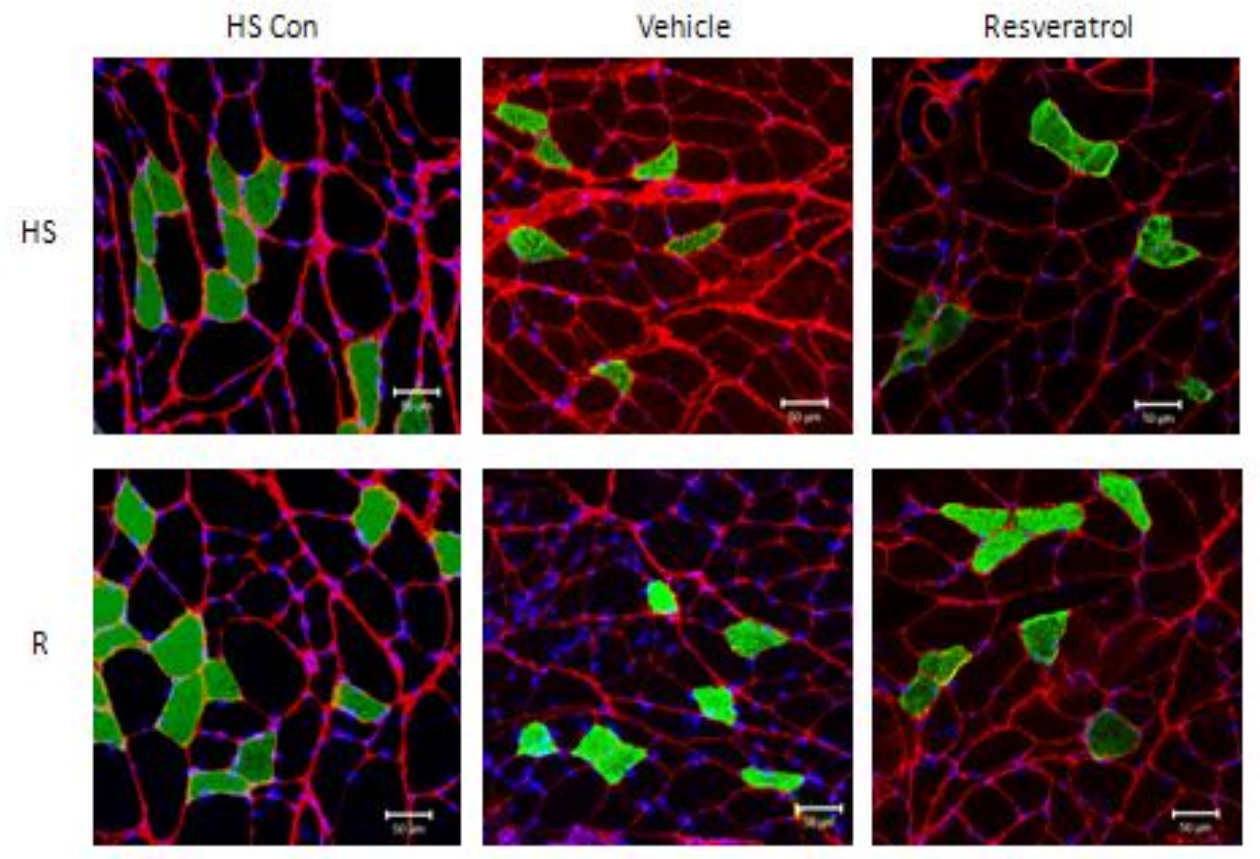

Figure 5

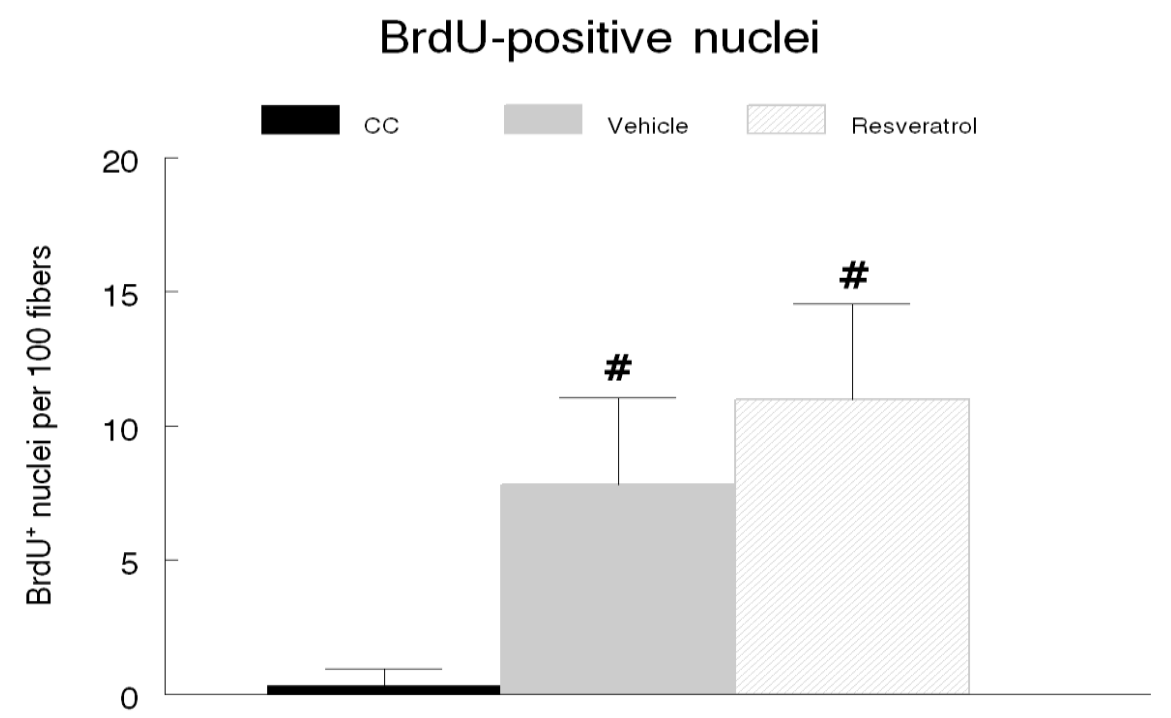


Figure 6A

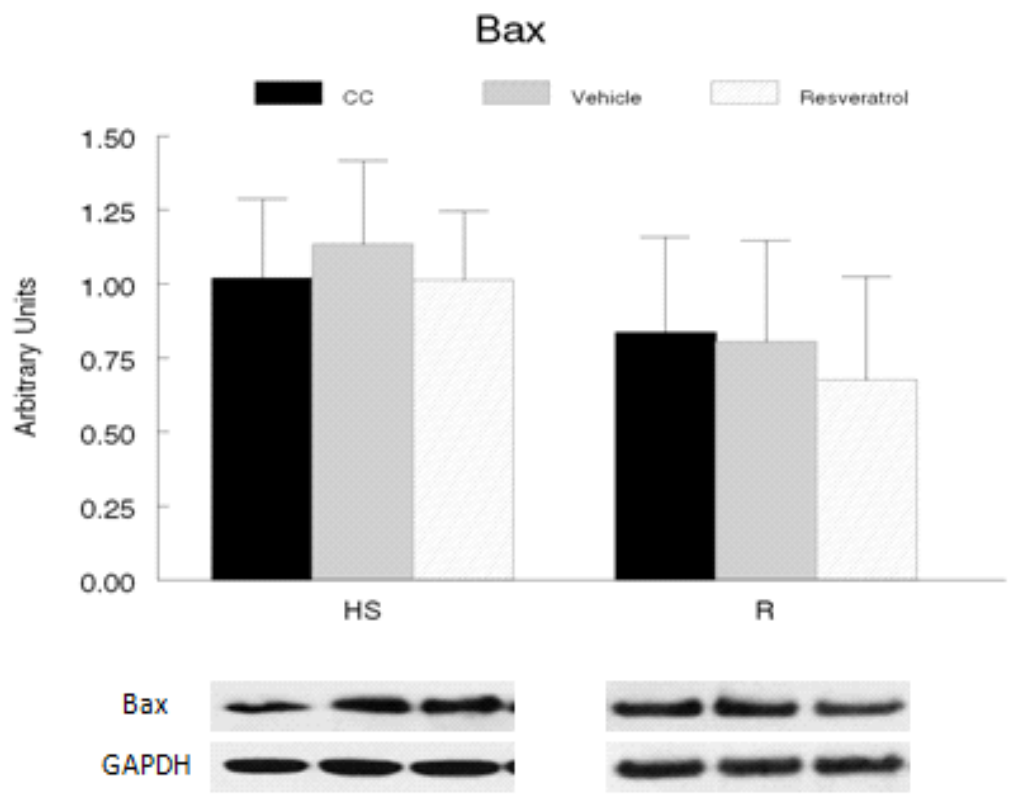

Figure 6B

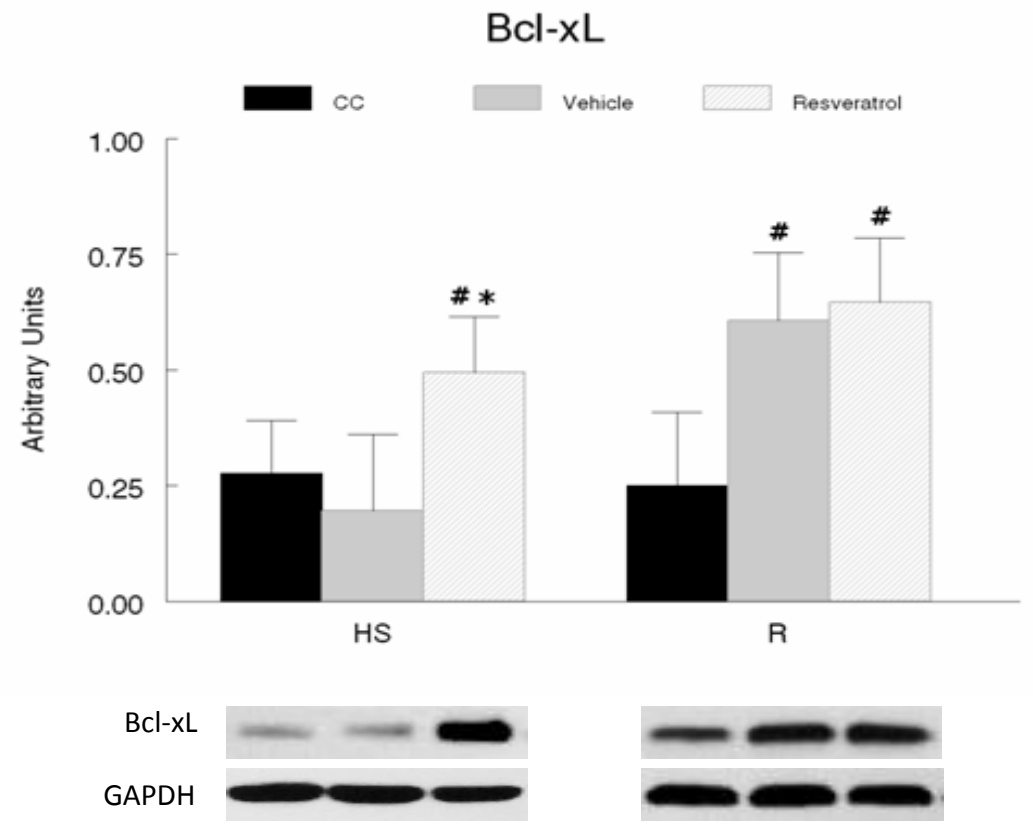


Figure 6C

TUNEL - HS

HS Con
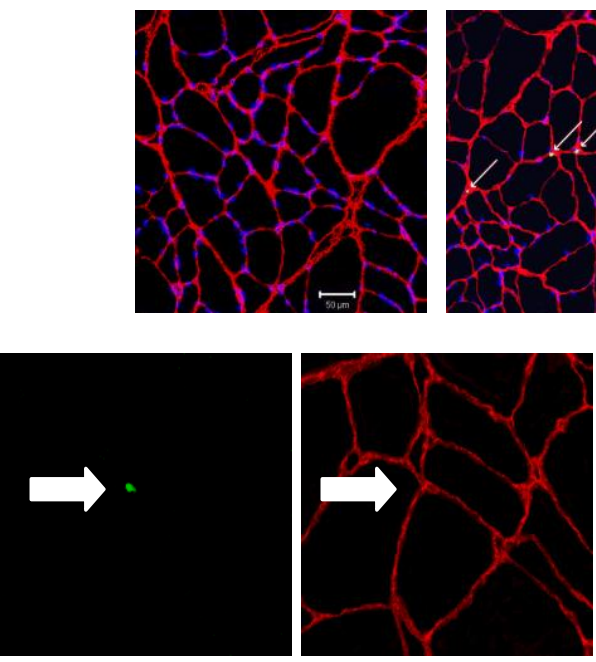

Vehicle
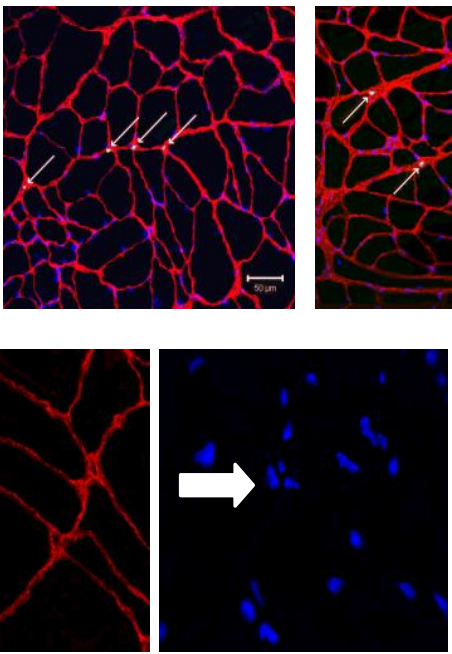

Resveratrol
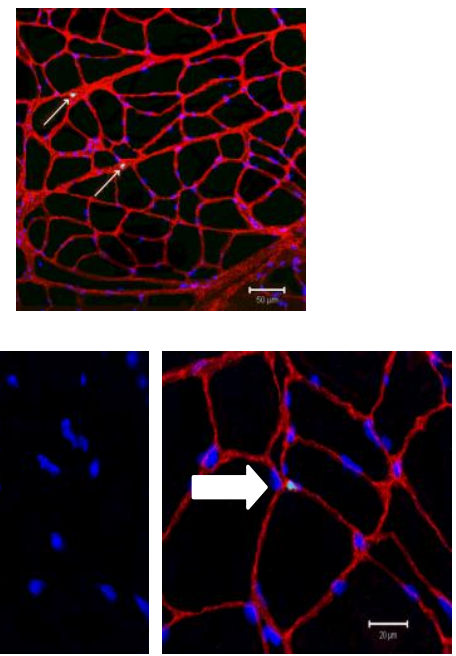

Figure 6D

TUNEL - R

R Con
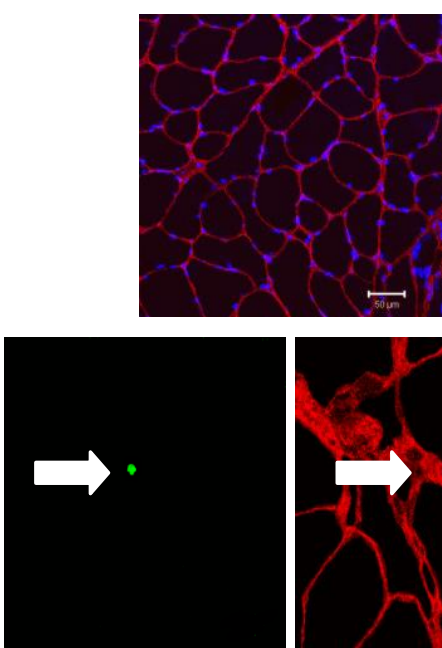

Vehicle

Resveratrol
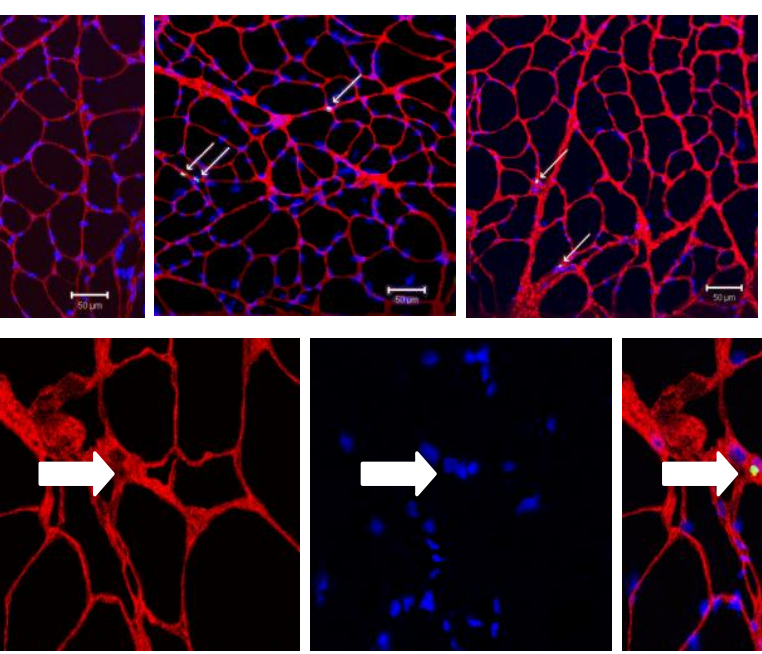

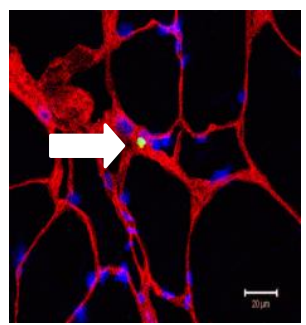


Figure 6E

Frequency of TUNEL Positive Nuclei

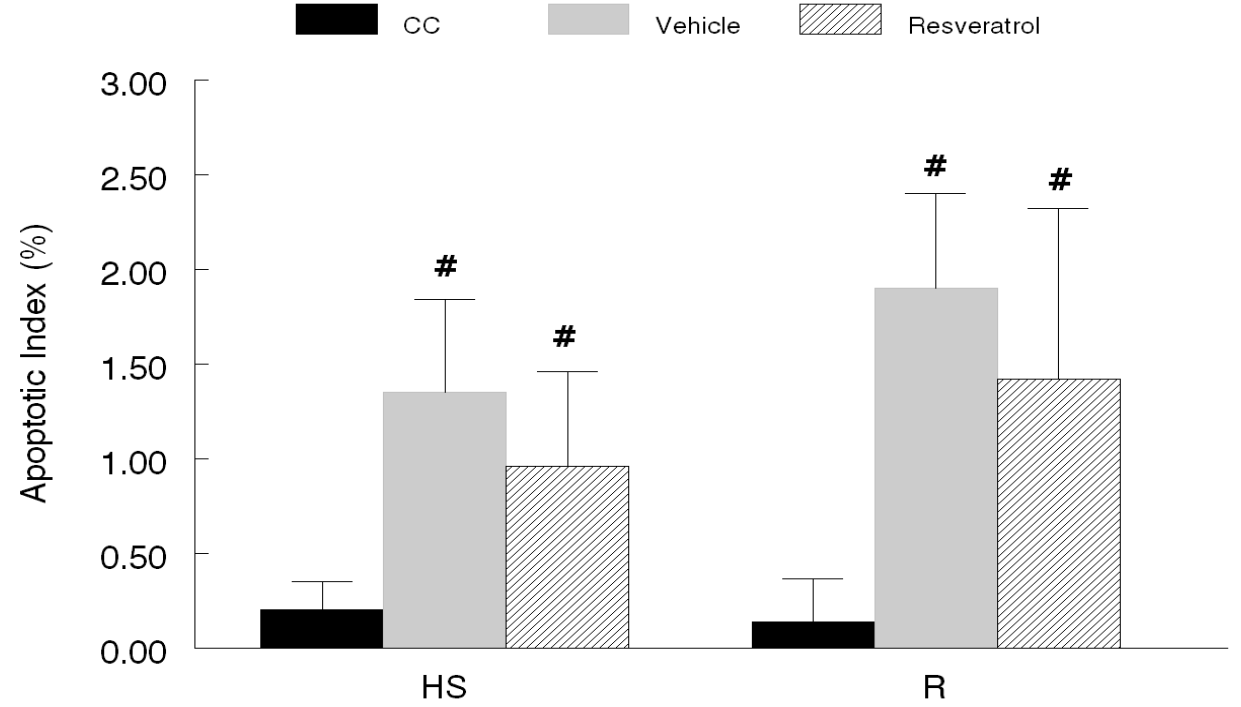




\title{
Chapter 4
}

\section{SIRT1 in the regulation of skeletal muscle regeneration following acute myotoxic injury}

\author{
Brian T. Bennett and Stephen E. Alway
}

Laboratory of Muscle Biology and Sarcopenia, Division of Exercise Physiology, West Virginia University School of Medicine, Morgantown, WV. 


\begin{abstract}
$\underline{\text { Abstract }}$
Silent mating type information regulator 2 homolog 1 (Sirt1), an $\mathrm{NAD}^{+}$-dependent histone deacetylase, has been implicated in the regulation of lifespan, tumorigenesis, aging, metabolism and gene expression. Recent work has also begun to uncover a possible role for Sirt1 in the regulation of skeletal muscle mass and function. We thereby utilized a cardiotoxin (CTX)induced muscle injury protocol to determine the effects of either the suppression or overexpression of Sirt1 on skeletal muscle regeneration in older-age (18-22 mo) transgenic animals. The left tibialis anterior (TA) muscle of all the animals were injected with CTX, while the right TA was injected with an identical volume of PBS and used as an intranimal control. Animals were then allowed to recover for a period of 3 or 7 days before both TA muscles were surgically removed and the animal was sacrificed. Sirt1 expression did not have an effect on muscle mass or fiber area in this model, which was not surprising considering it also did not alter satellite cell activity. However, increasing Sirt1 expression increased maximal tetanic force production in the regenerating muscles as compared to respective WT muscles which may indicate improved functional recovery in these muscles. Overall, these results suggest a slightly improved muscle recovery with increased Sirt1 expression. Future research should be conducted to determine if clinically modulated Sirt1 expression levels will prove beneficial in enhancing functional muscle regeneration in response to various perturbations.
\end{abstract}




\section{Introduction}

Silent mating type information regulator 2 homolog 1 (Sirt1) is a class III histone deacetylase (HDAC) which has been implicated in lifespan extension in several model organisms $(22,30,37)$. It is just one member of the mammalian family of sirtuins, which is composed of a total of seven proteins (Sirt1-7). Sirt1 is an NAD+-dependent deacetylase, increasing its activity in response to rising cellular $\mathrm{NAD}^{+} / \mathrm{NADH}$ ratios. Capable of deacetylating a variety of substrates, Sirt1 has been shown to play a role in an assortment of physiological activities, such as tumorigenesis, aging, metabolism, and the control of gene expression (43). Moreover, recent work has begun to uncover a beneficial role for Sirt1 expression in the maintenance of skeletal muscle mass and function (237), indicating a potential for improving regeneration in various muscle pathologies.

One of the key targets of Sirt1 in skeletal muscle is the transcriptional coactivator

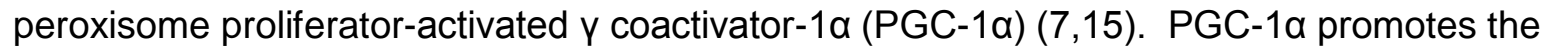
expression of genes related to mitochondrial biogenesis and oxidative metabolism in skeletal muscle, and its overexpression has been shown to increase fatigue resistance in muscles (25). PGC-1 $\alpha$ also appears to have a protective role in conditions of muscle atrophy. Transgenic mice overexpressing PGC-1 $\alpha$ in skeletal muscle experienced decreased denervation and fasting-induced muscle atrophy responses (208), and were protected from age-associated muscle wasting (249). This is most likely due to the reduced protein degradation that has been associated with PGC-1 $1 \alpha$ overexpression (33). Thus, the deacetylation of PGC-1 $\alpha$ by Sirt1 is one potential pathway for the involvement of Sirt1 in the regulation of skeletal muscle mass and function. Another downstream target of Sirt1 which may have a role in the regulation of skeletal muscle size and strength is NF-KB $(19,28)$. Activation of the NF-KB pathway alone has been shown to be sufficient to induce muscle wasting $(6,39)$. As Sirt1 activity inhibits the 
transactivation of NF-kB on the p65 subunit $(32,41)$, increasing Sirt1 may prove beneficial during periods of muscle wasting.

Recent research utilizing resveratrol, a known Sirt1 activator, has also provided insight into the possible benefits of Sirt1 in skeletal muscle maintenance. In one study, resveratrol inhibited dexamethasone-induced MuRF1 and atrogin-1 expression in cultured myotubes, and these effects were dependent on Sirt1 expression (1). Moreover, this study also provided evidence that resveratrol can attenuate increases in protein degradation as well as decreases in myotube diameter under these catabolic conditions (1). In another study, resveratrol supplementation significantly reduced decreases in both muscle weight and fiber area of animals subjected to a period of hindlimb unloading (26). Furthermore, resveratrol has been shown to increase muscle force under a variety of conditions $(13,21,23,34)$, providing evidence that Sirt1 may be beneficial to both skeletal muscle size and function, which could potentially translate into improved muscle regeneration.

While there are a variety of models available to study muscle regeneration, one of the most reproducible methods involves the injection of myotoxins, such as cardiotoxin (CTX), directly into the individual muscle to be studied $(12,16)$. CTX, a peptide isolated from snake venom, is a protein kinase C-specific inhibitor that appears to disrupt membrane organization, induce the depolarization and contraction of muscle cells, and has the ability to lyse various cell types (52). Utilizing this model, muscle degeneration has been observed to occur within one day of injection, mononuclear cell proliferation peaks 1-4 days following the injection, and myogenic cell differentiation and the formation of new myotubes occurs $\sim 5-6$ days following the CTX injection (52). Therefore, in this study we examined both Sirt1-overexpression $\left(\right.$ Sirt $\left.1^{+++}\right)$ and Sirt1-knockdown (Sirt1 ${ }^{-/}$) transgenic animals at days 3 and 7 post-injection in order to determine the potential effects of Sirt1 on skeletal muscle regeneration. It was hypothesized that Sirt1 overexpression would benefit muscle regeneration (i.e., strength and/or size), while reducing Sirt1 expression would be either detrimental or have no effect on regeneration. 


\section{Methods}

Animals. A total of 48 older-age (18-22 months) transgenic mice were used. Sirt1overexpressing $\left(\mathrm{Sirt}^{+/+}\right)$mice were generously obtained from Dr. Sadoshima (3). These mice were generated on an FVB background using the $\alpha$-myosin heavy chain ( $\alpha-\mathrm{MHC})$ promoter, which results in a mild overexpression of Sirt1 in fast fibers which contain $\alpha-M H C$. Muscle specific Sirt1-knockdown (Sirt $1^{-1}$ ) mice were generated by crossing 129-Sirt ${ }^{\text {tm } 1 Y g u} / \mathrm{J}$ (148) with a a-MHC cre mouse (Jackson labs, Cold Spring Harbor). This resulted in a knock down of Sirt1 in fast contracting fibers of skeletal muscle.

Twelve Sirt1-overexpressing $\left(\operatorname{Sirt1}{ }^{+/+}\right)$mice and twelve Sirt1-knockdown $\left(\right.$Sirt $\left.1^{-1 /}\right)$ mice were used to examine the effects of Sirt1 on muscle regeneration. In addition, twelve mutant Sirt1 $\left(\right.$ Sirt $\left.1^{+/}\right)$wild-type littermates were used as controls for each the Sirt $1^{+/+}$and Sirt $1^{-/-}$mice. All animals were housed at $20^{\circ} \mathrm{C}$ in barrier-controlled conditions under a $12: 12$ hour light-dark cycle. Proper animal care standards were followed by adhering to the recommendations for the care of laboratory animals as advocated by the American Association for Accreditation of Laboratory Animal Care and by following the policies and procedures detailed in the Guide for the Care and Use of Laboratory Animals as published by the U.S. Department of Health and Human Services and proclaimed in the Animal Welfare Act (PL89-544, PL91-979, and PL94279). All experimental procedures were approved by the Institutional Animal Care and Use Committee from the West Virginia University.

Bromodeoxyuridine administration. Five days prior to muscle injury, each animal received a 15 mg Bromodeoxyuridine (BrdU; 5-bromo-2'-deoxyuridine) pellet (21-day release; Innovative Research; Sarasota, FL) in order to analyze cellular proliferation. A small incision was made in the skin of the upper back of anesthetized (97\% Oxygen/3\% Isofluorane) animals whereupon the BrdU pellet was inserted subcutaneously. The incision was closed with a wound clip 
(MikRon Autoclip 9mm; Becton Dickinson; Sparks, MD) and the animal was allowed sufficient time to recover from anesthesia before being returned to its cage.

Muscle injury and collection. Cardiotoxin (CTX; from Naja Mossambica; Sigma) was prepared in sterile phosphate-buffered saline (PBS) at a concentration of $10 \mu \mathrm{M}$. The hind limbs of anesthetized mice were carefully shaved and $75 \mu \mathrm{L}$ of CTX was injected into the exposed tibialis anterior (TA) muscle of the left leg. Moreover, the TA muscle of the contralateral (i.e., right) leg was injected with $75 \mu \mathrm{L}$ of sterile PBS and was used as an intra-animal control. All mice were carefully monitored following this procedure to ensure a full recovery from anesthetization. Six animals from each group were then sacrificed either 3 or 7 days following the CTX injury. On the respective day of sacrifice, TA muscles were surgically removed from anesthetized (97\% Oxygen/3\% Isofluorane) animals, blotted to remove excess fluid, weighed, flash frozen in liquid nitrogen cooled isopentane, and stored at $-80^{\circ} \mathrm{C}$ for later analyses. Once the muscles had been excised, the animals were killed by removing their heart with surgical scissors.

In situ muscle physiological analyses. In situ isometric muscle contractile properties were examined in the TA muscles of the mice immediately prior to removing the muscles and sacrificing the animals. Mice were anesthetized ( $97 \%$ Oxygen/3\% Isofluorane) and placed on their right side on a heated $\left(37^{\circ} \mathrm{C}\right)$ plate. Utilizing surgical scissors, the distal tendons of all lower limb muscles except the TA were completely severed, ensuring that the TA was the only muscle which had control over any movements at the ankle joint. The foot of one leg was then secured to a footplate attached to a servomotor (Model 6350*358; Cambridge Technology Inc., Cambridge, MA). Dynamic Muscle Control (DMC) software (Aurora Scientific Inc., Aurora, Ontario, Canada) controlled the servomotor and maintained the correct angular position of the foot throughout. TA muscle contractions were stimulated using a High-Power Bi-Phase Current 
Stimulator (Aurora Scientific Inc.). Peak isometric tetanic force (Po) was performed as previously described (7) and force-frequency isometric force records were obtained by stimulating the muscle at $5,10,20,50,75$ and $100 \mathrm{~Hz}$, with 3 minutes of rest between each contraction. Once the first leg had finished, all distal muscle tendons from the lower contralateral leg except the TA were severed and the second leg was subsequently run through the aforementioned protocol. All contractile and fatigue measurements were analyzed off line using commercial software (DMI, Aurora Scientific).

Fiber cross-sectional area. Muscle fiber cross-sectional area (CSA) measurements were utilized to determine the extent of muscle fiber regeneration in the TA muscles of the CTXinjured and control muscles of the mice. CSA was determined by planimetry from three nonoverlapping regions of each tissue cross-section stained for lamina, utilizing a Zeiss LSM 510 Meta confocal microscope (Carl Zeiss Microimaging Inc.; Thornwood, NY) with a 10X objective. Mean myofiber CSA measurements were calculated with the Image J software $(\mathrm{NIH}$; Frederick, MD). Furthermore, we used these individual CSA measurements to determine the total relative area of each field which contained regenerating myofibers. To accomplish this, we used the sum area of all the regenerating myofibers within a given field, and expressed this value as a percentage of the total area of the respective field. Thus, we were able to analyze both the mean size of the regenerating fibers as well as the relative extent to which these fibers were regenerating throughout the whole TA muscle cross-section.

Fiber characteristics. Muscle fiber type analyses were performed in order to examine the development of various myosin heavy chain ( $\mathrm{MyHC}$ ) isoforms in the regenerating fibers. Frozen tissue cross sections measuring 8- $\mu \mathrm{m}$ thick from the mid-belly of the muscles were mounted on charged microscope slides (Fisher Scientific; Pittsburgh, PA) and stored at $-80^{\circ} \mathrm{C}$. Briefly, slides were air-dried 30 minutes, washed 10 minutes in PBS, and incubated 30 minutes with $4 \%$ 
bovine serum albumin (BSA) in PBS at room temperature (RT). Sections were then incubated overnight at $4^{\circ} \mathrm{C}$ with antibodies directed against laminin (MAB1914; Millipore; Temecula, CA) to visualize the basal lamina of each muscle fiber, as well as one of the following individual MyHC antibodies: BA-D5 (for MyHC I; Developmental Studies Hybridoma Bank; lowa City, IA), SC-71 (for MyHC IIA; DSHB), BF-F3 (for MyHC IIB; DSHB), and developmental MyHC (VP-M664; Vector Laboratories; Burlingame, CA). On the following day, slides were washed in PBS and incubated 1 hour at $37^{\circ} \mathrm{C}$ with secondary antibodies of donkey anti-rat rhodamine (712-025-150; Jackson ImmunoResearch Laboratories; West Grove, PA) to visualize the basal lamina and Alexa Fluor 488 (A10680; Invitrogen; Eugene, OR) to visualize the MyHC-positive fibers. The sections were then mounted with 4', 6-diamidino-2-phenylindole (DAPI) (Vectashield Mounting Medium; Vector Laboratories; Burlingame, CA) in order to visualize the myonuclei. Three images from non-overlapping regions of each tissue cross-section stained for individual MyHC's were used for fiber type proportions. All images were taken with a Zeiss LSM 510 Meta confocal microscope (Carl Zeiss) using a 10X objective. Fiber types were expressed as a proportion of MyHC-positive fibers to total muscle fibers for each individual MyHC examined.

BrdU detection. Frozen tissue cross sections measuring $8-\mu \mathrm{m}$ thick from the plantaris muscle was mounted on charged microscope slides (Fisher Scientific). Briefly, frozen tissue sections were air dried, fixed in methanol:acetone $(1: 1)$ at $-20^{\circ} \mathrm{C}$, and permeabilized with $0.4 \%$ Triton $\mathrm{X}$ 100 in PBS. Sections were then denatured using Reagent 2 and blocked with Reagent 3, both of which being components of the Calbiochem BrdU Immunohistochemistry System (HCS30; EMD Biosciences, Inc.; La Jolla, CA). Sections were incubated overnight in a biotinylated mouse anti-BrdU primary antibody provided in the Calbiochem kit. The tissue sections were then incubated with Fluorescein Avidin DCS (A-2011; Vector Laboratories, Inc.; Burlingame, CA) and subsequently blocked in normal goat serum. They were then incubated overnight in rat anti-lamina monoclonal (Millipore) antibody. Sections were incubated the following day with 
donkey anti-rat rhodamine conjugated (Jackson ImmunoResearch) secondary antibodies in a humidified chamber at $37^{\circ} \mathrm{C}$ for 1 hour in the dark. Finally, sections were washed in PBS and mounted with DAPI mounting medium (Vectashield) in order to visualize nuclei.

Immunofluorescence was visualized with a Zeiss LSM 510 Meta confocal microscope (Carl Zeiss). Images were taken from three non-overlapping regions of each tissue section with a 10X objective.

Identification of apoptotic nuclei. Frozen tissue cross sections measuring $8-\mu \mathrm{m}$ thick from the TA muscles were mounted on charged microscope slides (Fisher Scientific). Fluorescent labeling of terminal dUTP nick-end labeling (TUNEL) with lamina allowed the detection of apoptotic nuclei in the muscle sections. This was accomplished using a slight modification to the method previously reported for our lab (36). Briefly, the tissue sections were air dried, fixed with $4 \%$ paraformaldehyde, and permeabilized with $0.1 \%$ Triton X-100 in PBS for 30 minutes each at room temperature. The tissues were incubated overnight at $4^{\circ} \mathrm{C}$ in a rat anti-lamina monoclonal antibody (Millipore) to visualize the basal lamina of each muscle fiber. The sections were incubated the following day with donkey anti-rat rhodamine conjugated secondary antibody (Jackson ImmunoResearch), along with the TUNEL reaction mixture (11-684-795-910; Roche Diagnostics; Indianapolis, IN) in a humidified chamber at $37^{\circ} \mathrm{C}$ for 1 hour in the dark. The exclusion of the TdT enzyme in the TUNEL reaction mixture on one of the tissue sections on each slide was included as a negative control. The sections were mounted with DAPI (Vector Laboratories) and viewed using a 10X objective on the Zeiss LSM 510 Meta confocal microscope (Carl Zeiss). The numbers of TUNEL and DAPI-positive nuclei that were immediately adjacent to, or beneath the basal lamina were counted. Data is expressed as an apoptotic index, which is the quantity of TUNEL-positive nuclei per 100 myofibers. The apoptotic index was determined from three non-overlapping regions of each tissue cross section. 
Statistical analysis. The results are reported as means \pm standard deviation (SD).

Differences in means between groups were determined by multiple analysis of variance (MANOVA) Hotelling's T-Square test. Bonferroni post hoc analysis was performed between significant means. Chi-squared analyses was conducted between experimental groups for the fiber area-frequency data. A $p$-value of $<0.05$ was considered significant. 


\section{$\underline{\text { Results }}$}

Body and muscle weights. Throughout the experimental period, all animals appeared visibly healthy and neither the Sirt $1^{-/-}$nor Sirt $1^{+/+}$transgenic animals displayed any apparent phenotypic abnormalities. All animals were weighed immediately prior to muscle injury as well as on their respective day of sacrifice. No differences in bodyweight were observed between these two time points and therefore only the weights obtained on the day of sacrifice are presented in Table 1. On day 3 post-injury, there were no significant differences in bodyweight between the WT $(34.97 \pm 2.62 \mathrm{~g})$ and $\operatorname{Sirt1}^{-/}(36.46 \pm 6.57 \mathrm{~g})$ animals on the Sirt1 ${ }^{-/}$background. As expected, the CTX-injured TA muscle wet weights normalized to bodyweight were significantly reduced as compared to the control muscle of each individual animal. The normalized weights of these WT animals were $1.15 \pm 0.20 \mathrm{mg} / \mathrm{g}$ for the injured muscle, compared to $1.86 \pm 0.14$ $\mathrm{mg} / \mathrm{g}$ for the respective control muscle. The normalized weights for the Sirt $1^{-/-}$animals were $0.83 \pm 0.10 \mathrm{mg} / \mathrm{g}$ and $1.33 \pm 0.17 \mathrm{mg} / \mathrm{g}$ for the injured and control muscles respectively, both of which were significantly reduced as compared to each of the respective WT muscle values. However, when the values for the injured muscles were expressed as a percentage of the values for each individual animals' respective control muscles, this difference disappeared. The wet muscle weight of the injured muscles for the WT and Sirt $1^{-/-}$animals were $62.14 \pm 10.22 \%$ and $62.05 \pm 4.84 \%$ of the control muscles, respectively.

There were also no differences between the body weights of the WT $(32.53 \pm 1.95 \mathrm{~g})$ and $\operatorname{Sirt1}^{-/}(31.40 \pm 4.13 \mathrm{~g})$ animals on day 7 post-injury. The normalized wet weights from the injured muscles of both the WT $(1.21 \pm 0.12 \mathrm{mg} / \mathrm{g})$ and Sirt $1^{-/-}(0.83 \pm 0.10 \mathrm{mg} / \mathrm{g})$ animals were still significantly smaller than that of the control muscles, which were $1.90 \pm 0.20 \mathrm{mg} / \mathrm{g}$ and 1.37 $\pm 0.13 \mathrm{mg} / \mathrm{g}$ for the WT and Sirt $1^{-/}$animals, respectively. As with the animals on day 3 postinjury, both the injured and control muscles of the WT mice were significantly larger than their $\mathrm{Sirt}^{-/}$counterparts. However, expressing the injured muscles as a percentage of their 
respective control muscles in both the WT $(62.00 \pm 5.88 \%)$ and Sirt ${ }^{-/-}(60.98 \pm 8.35 \%)$ animals effectively eliminated these differences.

Of the animals on the Sirt $1^{+/+}$background, there were no differences in bodyweight between the WT and Sirt1 ${ }^{+/+}$animals at either day 3 or 7 post-injury. On day 3 , the bodyweights were $45.23 \pm 9.88 \mathrm{~g}$ and $45.98 \pm 14.25 \mathrm{~g}$ for the WT and Sirt $1^{+/+}$animals, respectively. Likewise, 7 days following injury the WT and Sirt $1^{+/+}$animals weighed $38.47 \pm 9.18 \mathrm{~g}$ and 45.23 $\pm 5.32 \mathrm{~g}$, respectively. The injured muscles of both the WT and Sirt $1^{+/+}$animals were significantly reduced at days 3 and 7 , as compared to their respective control muscles. However, no differences were observed in the normalized weights of the injured muscles between the two groups. On day 3 post-injury, the normalized muscle wet weights of the WT and $\operatorname{Sirt} 1^{+/+}$animals were $0.82 \pm 0.15 \mathrm{mg} / \mathrm{g}$ and $0.85 \pm 0.21 \mathrm{mg} / \mathrm{g}$, respectively. This is a significant decrease compared to the respective uninjured muscles of both the WT and Sirt $1^{+/+}$ animals, which were $1.28 \pm 0.14 \mathrm{mg} / \mathrm{g}$ and $1.32 \pm 0.29 \mathrm{mg} / \mathrm{g}$, respectively. On day 7 post-injury, the weights from the WT $(0.79 \pm 0.23 \mathrm{mg} / \mathrm{g})$ and Sirt $1^{+/+}(0.94 \pm 0.17 \mathrm{mg} / \mathrm{g})$ injured muscles were significantly smaller than that of the uninjured muscles, which were $1.30 \pm 0.16 \mathrm{mg} / \mathrm{g}$ and $1.35 \pm 0.17 \mathrm{mg} / \mathrm{g}$ for the WT and Sirt $1^{+/+}$animals, respectively.

Muscle force characteristics. Immediately prior to each individual animal being sacrificed, the in situ force characteristics of the TA muscles were obtained for analysis. We first examined the maximal tetanic force for both the injured and control muscles. These values were determined at a frequency of $100 \mathrm{~Hz}$ and all forces were normalized to the wet weight of the respective TA muscle the force was measured from. In the Sirt $1^{-/}$animals, no differences in normalized maximal tetanic force were found in the control muscles of the animals on day 3 post-injury, with values of $1.79 \pm 0.41 \mathrm{mN} / \mathrm{mg}$ and $2.32 \pm 0.66 \mathrm{mN} / \mathrm{mg}$ for the WT and Sirt $1^{-/-}$animals, respectively (Figure 1A). While there were no differences between them, the forces produced in both the injured WT $(0.51 \pm 0.35 \mathrm{mN} / \mathrm{mg})$ and $\mathrm{Sirt1}^{-/-}(0.90 \pm 0.46 \mathrm{mN} / \mathrm{mg})$ muscles were 
significantly smaller than those of their respective control muscles. On day 7 , these values remained largely consistent and once again, no differences were observed between the WT $(2.48 \pm 0.24 \mathrm{mN} / \mathrm{mg})$ and Sirt1 $1^{-/}(2.15 \pm 0.49 \mathrm{mN} / \mathrm{mg})$ control muscles (Figure 1B). The maximal forces produced in the injured muscles of both the WT $(0.74 \pm 0.25 \mathrm{mN} / \mathrm{mg})$ and Sirt1 $1^{-/}$ $(0.66 \pm 0.28 \mathrm{mN} / \mathrm{mg})$ animals were significantly smaller than the respective control muscle forces, however, there were no differences between these two groups of injured muscles. In the animals bred on the Sirt ${ }^{+/+}$background, no differences in normalized maximal tetanic force were observed on day 3 post-injury in the control muscles of the WT $(1.85 \pm 0.24$ $\mathrm{mN} / \mathrm{mg})$ and Sirt $1^{+/+}(1.79 \pm 0.43 \mathrm{mN} / \mathrm{mg})$ animals (Figure 1A). Whereas only the forces from the injured muscles of the WT $(0.42 \pm 0.17 \mathrm{mN} / \mathrm{mg})$ animals were significantly smaller than their respective control values, the maximal tetanic force from the Sirt $1^{+/+}(1.26 \pm 0.40 \mathrm{mN} / \mathrm{mg})$ animals was significantly greater than that of the WT animals.

On day 7 post-injury, the maximal force produced in the control muscles of the WT (1.36 $\pm 0.25 \mathrm{mN} / \mathrm{mg})$ and $\operatorname{Sirt}^{+/+}(1.52 \pm 0.31 \mathrm{mN} / \mathrm{mg})$ animals did not differ (Figure 1B). However, only the maximal force from the injured muscle of the WT $(0.64 \pm 0.40 \mathrm{mN} / \mathrm{mg})$ group was significantly smaller than its respective control muscle force, and the force from the injured muscle of the $\mathrm{Sirt}^{+/+}(1.24 \pm 0.58 \mathrm{mN} / \mathrm{mg})$ group did not differ from that of its' control muscles.

Next, we plotted a force-frequency curve of the muscle force data collected from both the control and injured TA muscles on days 3 and 7 post-injury. For this curve, muscle forces at a given frequency are expressed as a percentage of the force produced at $100 \mathrm{~Hz}$. In the animals bred on the Sirt $1^{-/}$background, the only differences in the force-frequency curve were observed on day 3 post-injury (Figure 2A). On this day, the injured muscle from the WT animals had significantly greater relative forces than their respective control muscles, as well as the injured muscles from the Sirt $1^{-/}$animals at frequencies of 5 and $10 \mathrm{~Hz}$. However, these differences did not persist into day 7 as there were no differences in relative forces at this time point. 
In the animals on the Sirt $1^{+/+}$background, there were no significant differences noted on day 3 post-injury at any of the frequencies, either between the groups of animals or within them (Figure $2 \mathrm{C}$ ). On day 7 , there was only one significantly different value observed. At a frequency of $75 \mathrm{~Hz}$, the injured muscles in the WT animals produced a significantly smaller relative force than did their respective control muscles (Figure 2D). No other differences were observed between or within any of the groups at any other frequency examined.

Muscle fiber morphology. TA muscles were analyzed both 3 and 7 days following muscle injury to track the progress of fiber regeneration. The mean CSA's of the control and regenerating myofibers were determined by planimetry from sections stained for lamina. In the Sirt ${ }^{-1 /}$ background groups, it is interesting to note that the mean CSA of the Sirt ${ }^{-1 /}$ control fibers were significantly $(p<.01)$ smaller than that of the WT control fibers at both 3 and 7 days postinjury (Figures 3A, 3B). On day 3 post-injury, the mean fiber CSA from the WT control muscles was $1,375.76 \pm 229.88 \mu \mathrm{m}^{2}$, as compared to $865.40 \pm 92.25 \mu \mathrm{m}^{2}$ from the Sirt1 ${ }^{-1}$ control muscles. However, although the mean fiber CSA from all of the injured muscles were significantly smaller than their respective control muscles, no differences were found between the injured WT and Sirt ${ }^{-1 /}$ muscles at either 3 or 7 days post-injury. On day 3 , the mean CSA from the injured WT fibers measured $163.50 \pm 43.25 \mu \mathrm{m}^{2}$, while the injured Sirt1 ${ }^{-/}$fibers measured $201.75 \pm 36.72 \mu \mathrm{m}^{2}$ (Figure $3 \mathrm{~A}$ ). On day 7 , the injured muscles continued to regenerate, enlarging to $428.89 \pm 92.25 \mu \mathrm{m}^{2}$ for the WT fibers and $332.64 \pm 93.13 \mu \mathrm{m}^{2}$ for the Sirt $1^{-1}$ fibers (Figure 3B).

In the Sirt $1^{+/+}$background animals, no differences in fiber CSA were noted between the control muscles from the WT and Sirt $1^{+/+}$animals at either day 3 or 7 post-injury. On day 3 , the fibers measured 1,400.17 $\pm 235.44 \mu \mathrm{m}^{2}$ and 1,405.40 $\pm 36.44 \mu \mathrm{m}^{2}$ in the WT and Sirt1 ${ }^{+/+}$control muscles, respectively (Figure 3A). On day 7, these values were 1,149.90 $\pm 177.64 \mu \mathrm{m}^{2}$ and $1,149.07 \pm 177.64 \mu \mathrm{m}^{2}$ for the WT and Sirt1 ${ }^{1 /+}$ animals, respectively (Figure 3B). As expected, 
the regenerating fibers from both the WT and Sirt $1^{+/+}$animals were significantly smaller than their respective control muscle fibers both 3 and 7 days following the CTX administration. However, there were no differences between the WT and Sirt $1^{+/+}$animals' mean fiber CSA from the injured muscles on either of these days. On day 3 , the mean fiber CSA from the injured muscles of the WT and Sirt $1^{+/+}$animals measured $301.53 \pm 111.42 \mu \mathrm{m}^{2}$ and $257.93 \pm 11.54$ $\mu \mathrm{m}^{2}$, respectively (Figure $3 \mathrm{~A}$ ). By day 7 post-injury, these measurements increased to $373.10 \pm$ $99.26 \mu \mathrm{m}^{2}$ and $408.30 \pm 116.65 \mu \mathrm{m}^{2}$ for the WT and Sirt $1^{+/+}$animals, respectively (Figure 3B).

Next, we examined the relative area within the TA muscle belly which actually contained regenerating myofibers in order to gain a fuller understanding as to the extent of muscle regeneration than what could be obtained with the mean myofiber CSA measurements alone. To accomplish this, we expressed the sum of all of the myofiber CSA's in a given field as a percentage of the total area of this field from which these fibers were measured. In the Sirt $1^{-1-}$ background animals on day 3 post-injury, it was interesting to note that the control muscles of the Sirt $1^{-/-}(63.44 \pm 7.80 \%)$ animals had significantly less relative myofiber area per field than did their respective WT $(75.81 \pm 11.13 \%)$ counterparts (Figure $3 \mathrm{C})$. Although they encompassed a significantly smaller percentage of myofiber area per field than the respective control muscles, no differences were found between the injured muscles from the WT $(9.54 \pm 3.74 \%)$ and Sirt $1^{-/}$ $(14.26 \pm 4.44 \%)$ animals on day 3 . On day 7 , the control muscles of the Sirt $1^{-/}(59.04 \pm$ $19.48 \%$ ) animals maintained a smaller percentage of myofiber area per field than did the WT (71.03 $\pm 13.23 \%)$ group, although here it did not reach statistical significance (Figure 3D). Similar to day 3 post-injury, both the WT $(26.15 \pm 6.68 \%)$ and Sirt1 ${ }^{-/}(19.25 \pm 6.65 \%)$ groups had significantly reduced total myofiber areas than did their respective control muscles, although no differences were found between these regenerating muscles on day 7 .

In the Sirt $1^{+/+}$groups, no differences were found in the total myofiber area per field between the WT $(59.09 \pm 21.70 \%)$ and $\operatorname{Sirt}^{+/+}(58.72 \pm 15.37 \%)$ groups on day 3 post-injury (Figure 3C). As expected, both the WT (17.10 $\pm 5.10 \%)$ and Sirt1 ${ }^{+/+}(7.81 \pm 2.61 \%)$ groups had 
significantly reduced total myofiber areas per field in the injured muscles as compared to their respective control muscles. Moreover, it was determined that these regenerating muscles in the Sirt $1^{+/+}$animals had a significantly $(\mathrm{p}<.01)$ smaller total myofiber area per field than did the WT group. On day 7 post-injury, no differences existed between the control muscles of the WT $(67.36 \pm 13.82 \%)$ and Sirt $^{+/+}(66.32 \pm 10.34 \%)$ animals (Figure 3D). Although both the injured WT $(34.20 \pm 6.48 \%)$ and Sirt $1^{+/+}(25.29 \pm 10.58 \%)$ muscles had a significantly smaller total myofiber area than that of their respective control muscles, no differences were observed between the regenerating muscles of these two groups.

Fiber composition. Both injured and control muscles were examined to determine the relative fiber MyHC composition of each at the different time points following muscle injury. In the Sirt $1^{-/-}$ animals, the composition of the regenerating fibers was very similar to that of the injured WT muscles both 3 and 7 days post-injury (Figures 4A, 4B). The regenerating muscles of each of these animals consisted of a low overall percentage of type I, IIA, and IIB MyHC fibers, while the great majority of these tested positive for developmental fibers. However, there was one difference to note, which occurred on day 7. Upon examination of the type IIB fibers, it was determined that the injured muscles of the WT $(34.1 \pm 6.1 \%)$ animals had significantly more of these fibers than did the Sirt $1^{-/}(6.1 \pm 3.0 \%)$ animals (Figure 4B). No other differences in fiber composition were observed in these animals.

In the Sirt ${ }^{+/+}$background, the same general pattern repeated itself. On both days 3 and 7 post-injury, the only differences observed between the injured muscles of the Sirt $1^{+/+}$and WT animals were in the type IIB MyHC fiber composition. On day 3, the relative composition of type IIB fibers was significantly greater in the WT $(4.1 \pm 2.1 \%)$ compared to the Sirt $1^{+/+}$animals, which did not have any of these fibers at this stage (Figure 4C). This gap widened on day 7 post-injury, with the composition of type IIB fibers remaining low in the Sirt $1^{+/+}(16.8 \pm 7.7 \%)$ animals, while it returned to that of the control muscles in the WT $(43.0 \pm 9.8 \%)$ animals (Figure 
4D). As with the animals on the Sirt $1^{-1}$ background, the only differences observed in fiber composition were in the type IIB fibers.

Satellite Cells. Satellite cells are an integral component of proper skeletal muscle regeneration. Therefore, we examined several satellite cell markers at both days 3 and 7 postinjury. Implanting a BrdU pellet into each of the animals five days prior to muscle injury allowed us to track newly synthesized satellite cells throughout the regeneration period. As expected, the control muscles from all of the animals had minimal BrdU incorporation, as satellite cells remain quiescent under basal conditions. However, BrdU incorporation was significantly increased in all of the injured muscles, as satellite cells were activated in these muscles in an attempt to repair the damaged fibers.

On day 3 post-injury, the quantity of BrdU-positive nuclei per 100 fibers was not different in the TA of Sirt1 ${ }^{-1}(4.30 \pm 1.33)$ animals than it was in their WT $(2.67 \pm 1.05)$ counterparts (Figure 5A). Likewise, the injured muscles from the Sirt1 ${ }^{+/+}(2.44 \pm 1.44)$ animals had approximately the same proportion of BrdU-positive nuclei as their respective WT (4.64 \pm 1.85$)$ littermates. On day 7 post-injury, the injured muscles of the Sirt $1^{-1-}(3.90 \pm 2.27)$ animals once again were not different than the WT $(2.09 \pm 1.00)$ animals (Figure 5B). On the Sirt $1^{+/+}$ background, there were no differences noted between the injured muscles of the WT (2.76 \pm $1.60)$ and $\operatorname{Sirt} 1^{+/+}(2.18 \pm 1.48)$ animals.

Apoptosis. To determine if apoptosis played a role in hindering muscle regeneration we performed a TUNEL assay, which detects DNA strand breaks generated during apoptosis. We expressed the results of the assay in a TUNEL index, namely, the quantity of TUNEL-positive nuclei per 100 myofibers. There was a significant increase in apoptotic nuclei in the regenerating myofibers of all the animals. On the Sirt ${ }^{-1 /}$ background on day 3 post-injury, the TUNEL index of the WT animals increased from $0.14 \pm 0.10$ in the control muscles to $3.99 \pm$ 
2.03 in the injured muscles (Figure 6A). Likewise, the TUNEL index increased from $0.32 \pm 0.24$ in the control muscles to $3.18 \pm 2.29$ in the injured muscles of the Sirt $1^{-/-}$animals. However, no differences were determined to exist between either the control or injured muscles of the WT and $\mathrm{Sirt1}^{-/-}$groups.

On day 7 , the same pattern appeared as with day 3. Muscles from the WT animals increased their respective TUNEL index from $0.04 \pm 0.08$ in the control muscles to $4.18 \pm 1.40$ in the injured muscles (Figure 6B). Similarly, the index was $0.22 \pm 0.21$ in the control muscles of the Sirt $1^{-/}$animals, and increased to $3.82 \pm 1.56$ in the injured muscles of these animals. Once again, there were no differences noted when comparing the control and injured muscles of the WT animals with that of the respective muscles from the Sirt $1^{-/}$animals.

In the animals bred on the Sirt $1^{+/+}$background, there were no differences in the TUNEL index between the WT $(0.08 \pm 0.13)$ and Sirt $1^{+/+}(0.05 \pm 0.10)$ control muscles on day 3 postinjury (Figure 6A). Moreover, although each groups' index was significantly increased from the respective control muscles, there were no differences between the injured muscles of the WT $(3.93 \pm 1.55)$ and $\operatorname{Sirt1}^{+/+}(2.60 \pm 0.90)$ groups. These trends persisted into day 7 post-injury. The index from the control muscles of the WT animals on this day increased from $0.03 \pm 0.09$ to $3.04 \pm 1.64$ in the injured muscles (Figure 6B). In the Sirt $1^{+/+}$animals, the TUNEL index was found to be $0.03 \pm 0.08$ in the control muscles, increasing to $3.21 \pm 1.89$ in the injured muscles. However, there were no differences observed between the WT and Sirt $1^{+/+}$groups when comparing their respective control and regenerating muscles. 


\section{Discussion}

Sirt1, the mammalian homolog of the yeast Sir2, has been implicated in lifespan extension, neurodegenerative and cardiovascular disease, cancer, and certain metabolic disorders (107). However, the role of Sirt1 in the maintenance and regeneration of skeletal muscle is much less understood. Here we utilized a CTX-induced muscle injury model to examine the effects of Sirt1 on skeletal muscle regeneration in older-age transgenic Sirt1 animals.

\section{Sirt1 has minimal effects on muscle mass during regeneration}

On both days 3 and 7 post-injury, there was no difference in the bodyweights of either the Sirt ${ }^{-1-}$ or Sirt $1^{+/+}$animals and their respective WT counterparts. As expected (52), the CTX caused considerable damage to the TA muscles within a short time, as evidenced by significantly smaller normalized muscle wet weights observed 3 days following the injury protocol. It was interesting to note that both the injured and control muscles from the Sirt $1^{-/}$ animals had significantly smaller normalized wet muscle weights than the respective WT muscles on both days 3 and 7 post-injury. However, the injured and control muscle weights of the Sirt ${ }^{-1 /}$ animals did not differ from the values of those in the respective muscles of the Sirt $1^{+/+}$ and WT animals bred on the Sirt $1^{+/+}$background. Moreover, when the injured muscles were expressed as a proportion to the weight of the control muscles the differences were nullified, suggesting that the injured muscles of the Sirt ${ }^{-1}$ animals regenerated as well as the WT muscles in terms of wet muscle weight. In the animals bred on the Sirt $1^{+/+}$background, there were no differences observed in the normalized wet muscle weights between the WT and Sirt $1^{+/+}$animals on either day 3 or 7 . Therefore, Sirt1 may have a very limited impact on the overall mass of regenerating myofibers. It is recognized that both Sirt1 knockdown and overexpression occurred in only the fast contracting fibers, and while the majority of fibers in the 
TA muscle were composed of fast fibers, a small percentage of type I fibers would have been unaffected by the genetic manipulation. It is not clear from this study if knockdown or overexpression of Sirt1 in all muscle fibers would have altered the overall conclusions, but this bears testing in future studies.

\section{Sirt1 overexpression increases force in regenerating muscles}

Next, we examined various force characteristics to determine any effects Sirt1 could possibly have on the contractile properties of regenerating muscles. We first examined the maximal tetanic forces, which were normalized to the wet weight of each individual TA muscle being examined. As expected with muscle injury $(11,27)$, the force output of the injured muscle was less than that of the control muscle. This was true even after normalizing all of the forces to the wet weight of each individual muscle. In the animals on the Sirt $1^{-/-}$background, there were no differences between the normalized force from either the control or injured muscles when comparing the WT and Sirt $1^{-/}$animals at either day 3 or 7 post-injury. However, the forces from the injured muscles were significantly smaller than that of the control muscles for each individual animal, indicating that the CTX treatment generated functional deficits in the muscles. Based on our data collected from the animals bred on the Sirt $1^{+/+}$background, it appears that overexpression of Sirt1 may improve functional muscle regeneration, providing a quicker recovery of strength during the initial stages of recovery.

On day 3 post-injury, both the injured muscles from the WT and Sirt $1^{+/+}$animals produced significantly less force than their respective control muscles. However, the mean normalized tetanic force of the injured Sirt $1^{+/+}$muscles was three-fold greater than that of the injured WT muscle. On day 7 , the tetanic force of the Sirt $1^{+/+}$muscles remained consistent with that observed on day 3. At this time point, the tetanic force from these muscles was no longer significantly smaller than that of the respective control muscles, although the mean was still slightly reduced. However, the force produced in the injured muscles of the Sirt $1^{+/+}$animals was 
significantly greater than that of the injured WT muscles, which again produced significantly less force than their respective control muscles.

While our study suggests that decreasing Sirt1 expression does not have an effect on force production during muscle regeneration, our findings that upregulating its expression can increase muscle force is in agreement with several previous studies. In these studies $(13,21,23,34)$, it was shown that supplementation with resveratrol, a Sirt1 activator, provided significant benefits in skeletal muscle force production under a variety of physiological conditions. Thus, the results from our present study offer additional support for the positive role of increasing Sirt1 expression on muscle force. Moreover, the neutral effect on muscle force we observed when decreasing the expression of Sirt1 suggests that while it may not be important for muscle function during normal regeneration, increasing Sirt1 expression may provide a shortened functional recovery following muscle injury.

We next analyzed a force-frequency curve to examine additional contractile properties in the regenerating muscles of the transgenic animals. On day 3 post-injury, the only significant differences that occurred were in the animals on the Sirt ${ }^{-/}$background. At 5 and $10 \mathrm{~Hz}$, the injured WT muscles had a force-frequency curve which was shifted significantly to the left of both their respective control muscles and the injured muscles of the Sirt $1^{-/-}$animals. This leftward shift in the force-frequency curve is indicative of a slower muscle phenotype. However, this leftward shift was abolished by day 7 and there were no differences between any of the force-frequency curves plotted on this day. In the animals on the Sirt $1^{+/+}$background, the forcefrequency curves of all the muscles examined remained largely consistent on both days 3 and 7. The regenerating muscles from the Sirt $1^{+/+}$animals had curves at both days 3 and 7 postinjury which were no different from what was observed in the respective control muscles or in any of the WT muscles. Thus, no clear phenotypic changes were observed in the muscle contractile properties of the Sirt $1^{+/+}$animals. 
It was interesting to note that none of our force-frequency curves uncovered any significant differences between the WT animals and either of the Sirt1 transgenic animal groups. In a recent study by Jackson et al. (20), administration of resveratrol to aged animals showed a rightward shift in a force-frequency curve. This would suggest a shift to a fast-muscle phenotype and would provide additional support for the increased maximal tetanic force production we observed in our Sirt $1^{+/+}$animals. However, we saw no such shift in the forcefrequency curves from these animals in our present study. One possible explanation of the Jackson et al. findings is that the force-frequency curve shift was observed in aged animals under homeostatic conditions, unlike our present study in which there was a significant perturbation applied to the muscles to induce regeneration. Moreover, it is plausible that the muscles in our animals were not recovered to an extent where any possible differences in the force-frequency curves could emerge.

\section{Sirt1 in muscle fiber regeneration}

In order to determine how well the different Sirt1 muscles regenerated, we examined both the mean CSA of the fibers as well as the relative area within the muscle belly which was filled with myofibers. When examining the mean CSA's, it was interesting to note that while there were no differences between the area of the regenerating WT and Sirt $1^{-/}$muscles, the control muscles of the Sirt1 ${ }^{-1}$ animals were significantly smaller than those of the WT animals at both days 3 and 7 post-injury. These findings are consistent with what we observed when calculating the relative area within a muscle fiber which contained myofibers as well. There were no differences between the injured WT and Sirt $1^{-/}$muscles at either day 3 or 7 post-injury. However, the relative area of the control muscles which contained myofibers was significantly less in the Sirt $1 \%$ animals than in the WT animals at day 3 . While these values did not reach statistical significance on day 7 , the total relative myofiber area of the Sirt ${ }^{-1-}$ animals was still smaller than that of the WT animals. This finding may be partly explained by recent research in 
which it was observed that Sirt1 activity and expression were downregulated in various models of muscles wasting $(2,31)$. While our model was very much different than that of muscle wasting, these combined observations may allow for the speculation that there is a potential role of Sirt1 expression in general skeletal muscle maintenance.

In the animals bred on the Sirt $1^{+/+}$background, no such differences in the control muscles existed between the two groups in either mean CSA or relative total myofiber area. On both days 3 and 7 post-injury, the mean CSA from the injured muscles of the Sirt $1^{+/+}$animals was no different than the WT muscles. In contrast, the relative total myofiber area of the Sirt $1^{+/+}$ muscles was significantly less than that of the WT muscles on day 3. However, this difference between the injured muscles was eliminated by day 7 post-injury. This was an unexpected finding, given that several studies utilizing the Sirt1 activator resveratrol have suggested that Sirt 1 is able to protect skeletal muscle from several catabolic conditions $(10,18,26,35)$. The results from these studies are likely attributed to the ability of Sirt1 to attenuate increases in protein degradation and the expression of the atrophy-related ligases MuRF1 and atrogin-1 during catabolic conditions (1). Thus, the results from these prior studies in combination with our present data appear to suggest that although Sirt1 may be beneficial during periods of skeletal muscle catabolism by attenuating proteolysis and the expression of atrophy-related ligases, it may not have any significant effects on the early hypertrophy of regenerating muscles.

Furthermore, we determined the fiber type proportions of each of the muscles. The largest proportion of fiber types in the regenerating muscles of all groups was developmental fibers. This was expected at this early stage of recovery, as the muscles did not have enough time to convert to full-fledged MyHC fiber types. However, no differences existed between any of the groups in the proportion of developmental fiber types at either 3 or 7 days post-injury. Furthermore, gain or loss of Sirt1 expression had no effect on either type I or IIA MyHC fiber composition. Surprisingly, both the Sirt $1^{+/+}$and Sirt $1^{-/}$animals had a smaller proportion of type 
IIB fibers in the injured muscles than the injured muscles of their respective WT mice. It therefore is likely that Sirt1 plays a role in the regeneration of fast-twitch MyHC fibers, although the mechanism is unclear at this time.

\section{Satellite cells}

Satellite cells are skeletal muscle stem cells that are activated when there is a sufficient perturbation to the tissue. In order to repair the damaged tissue, satellite cells proliferate, differentiate, fuse to existing myofibers, and then begin to hypertrophy in an attempt to restore muscle fiber size and function. In a 2003 study by Fulco et al. (14), it was suggested that Sirt1 may inhibit differentiation through the repression of the myogenic transcription factor MyoD. However, decreasing levels of $\mathrm{NAD}^{+}$during differentiation may decrease Sirt1 levels, thereby allowing normal differentiation to proceed (14). Moreover, Sirt1 has been shown to increase the proliferation of satellite cells (198), possibly providing an increased stimulus for effective fiber regeneration.

In the present study, we did not see an increase in BrdU-labeled nuclei in the animals overexpressing Sirt1 at either 3 or 7 days post-injury. Likewise, decreased Sirt1 expression in the Sirt $1^{--}$animals had no effect on the amount of BrdU labeling observed in the regenerating muscles of these animals as compared to the injured WT muscles. The lack of any differences in the injured muscles from either the Sirt $1^{+/+}$or Sirt $1^{-/-}$animals cannot be explained by the loss or protection of myonuclei from apoptosis as we did not observe any variance in apoptotic signaling determined via TUNEL staining. Thus, our in vivo model could neither confirm nor deny the previous findings of the effects of Sirt1 expression on satellite cell function observed under in vitro conditions. Therefore, further in vivo work needs to be completed before any conclusions may be made on what role, if any, Sirt1 expression has on satellite cell activity and function in muscle regeneration. 


\section{Future directions}

Our present study was novel in that it attempted to examine the effects of manipulating Sirt1 expression on skeletal muscle regeneration in an in vivo model of muscle injury. Although Sirt1 did not play a role in muscle mass or fiber area during regeneration, increasing its' expression imparted beneficial effects to the force characteristics of the muscle. The increased force production which coincided with elevated Sirt1 expression may translate to improved functional muscle recovery following injury or disruptions in normal muscle homeostasis in clinical populations. Therefore, further work in which Sirt1 expression is increased experimentally should be investigated in order to evaluate its efficacy in treating various clinical populations such as the elderly in response to extended periods of disuse. Moreover, our study failed to show a correlation between Sirt1 expression and satellite cell activity. It is presently unknown if Sirt1 has an effect on this activity at the organism level, as previous research into this area has only involved in vitro work. Future work specifically aimed at this utilizing in vivo models may provide a more comprehensive assessment of any potential effects of Sirt 1 on satellite cell activity.

Our overall results from this study indicate that although Sirt1 expression may not affect muscle morphology, its effects on force production may be useful in treating various conditions which require muscle regeneration. It would be clinically valuable if Sirt1 is able to provide a shortened functional recovery in response to muscle perturbations. Therefore, or current findings may be useful as a base for further exploration into the potential of Sirt1 as an effective clinical tool used to enhance skeletal muscle regeneration. 


\section{Table Legends}

Table 1. Bodyweight and muscle wet weight measurements from each of the genotypes at both 3 and 7 days post-injury. ${ }^{*} \mathrm{P}<.05$ vs. respective injured $\mathrm{WT}, \# \mathrm{P}<.05$ injured $v s$. respective control, $\uparrow \mathrm{P}<.05$ vs. respective $\mathrm{WT}$ control. 


\section{Figure Legends}

Figure 1. $A, B)$ The maximal tetanic force of each genotype was examined at both days $3(A)$ and $7(B)$ post-injury. Individual forces were normalized to the muscle weights of each animal. ${ }^{*} \mathrm{P}<.05$ vs. injured WT, \# $\mathrm{P}<.05$ injured vs. respective control.

Figure 2. $A, B) \mathrm{A}$ force-frequency curve was developed for the Sirt $1^{-/-}$animals at both $3(A)$ and 7 (B) days post-injury. $C, D)$ A force-frequency curve was developed for the $\operatorname{Sirt} 1^{+/+}$animals at both $3(C)$ and $7(D)$ days post-injury. Values are the force produced at each given frequency expressed as a percentage of the force produced at $100 \mathrm{~Hz}$ for each individual animal. ${ }^{*} \mathrm{P}<.05$ injured vs. injured at given frequency, \# $\mathrm{P}<.05$ vs. respective control at given frequency.

Figure 3. Mean myofiber cross-sectional area (CSA) and total myofibril area analysis were performed for each genotype. $A, B$ ) Fiber CSA was determined by planimetery on both days 3 $(A)$ and $7(B)$ post-injury. $C, D)$ Total myofibril area is defined as the total area within a given field that contains myofibers and was determined both $3(C)$ and $7(D)$ days post-injury in each of the groups. ${ }^{*} \mathrm{P}<.05$ vs. injured $\mathrm{WT}, \# \mathrm{P}<.05$ injured vs. respective control, $\dagger \mathrm{P}<.05$ vs. WT control.

Figure 4. The MyHC fiber composition of both the injured and control TA muscles were analyzed for each group of animals. $A, B) \mathrm{MyHC}$ fiber composition was determined for the Sirt $1^{-/}$animals both $3(A)$ and $7(B)$ days post-injury. $\left.C, D\right)$ MyHC fiber composition of the Sirt ${ }^{+/+}$animals was determined at both $3(C)$ and $7(B)$ days post-injury. ${ }^{*} \mathrm{P}<.05$ injured $v s$. injured at respective fiber type. 
Figure 5. $A, B)$ BrdU incorporation was determined in all of the animals at both $3(A)$ and $7(B)$ days post-injury. Values are expressed as the quantity of BrdU-positive myonuclei per onehundred fibers. \# $\mathrm{P}<.05$ injured vs. respective control.

Figure 6. $A, B)$ Apoptosis was assessed via TUNEL staining in all of the animals at both $3(A)$ and $7(B)$ days post-injury. The TUNEL index is expressed as the quantity of TUNEL-positive myonuclei per one-hundred fibers. \# $\mathrm{P}<.05$ injured vs. respective control. 
Reference List

1. Alamdari N, Aversa Z, Castillero E, Gurav A, Petkova V, Tizio S and Hasselgren PO. Resveratrol prevents dexamethasone-induced expression of the muscle atrophy-related ubiquitin ligases atrogin-1 and MuRF1 in cultured myotubes through a SIRT1-dependent mechanism. Biochem Biophys Res Commun 417: 528-533, 2012.

2. Alamdari N, Smith IJ, Aversa Z and Hasselgren PO. Sepsis and glucocorticoids upregulate p300 and downregulate HDAC6 expression and activity in skeletal muscle. Am J Physiol Regul Integr Comp Physiol 299: R509-R520, 2010.

\section{Alcendor RR, Gao S, Zhai P, Zablocki D, Holle E, Yu X, Tian B, Wagner T, Vatner SF and} Sadoshima J. Sirt1 regulates aging and resistance to oxidative stress in the heart. Circ Res 100: 15121521, 2007.

4. Alway SE. Attenuation of $\mathrm{Ca}(2+)$-activated ATPase and shortening velocity in hypertrophied fast twitch skeletal muscle from aged Japanese quail. Exp Gerontol 37: 665-678, 2002.

5. Brault JJ, Jespersen JG and Goldberg AL. Peroxisome proliferator-activated receptor gamma coactivator 1alpha or 1 beta overexpression inhibits muscle protein degradation, induction of ubiquitin ligases, and disuse atrophy. J Biol Chem 285: 19460-19471, 2010.

6. Cai D, Frantz JD, Tawa NE, Jr., Melendez PA, Oh BC, Lidov HG, Hasselgren PO, Frontera WR, Lee J, Glass DJ and Shoelson SE. IKKbeta/NF-kappaB activation causes severe muscle wasting in mice. Cell 119: 285-298, 2004. 
7. Canto C, Jiang LQ, Deshmukh AS, Mataki C, Coste A, Lagouge M, Zierath JR and Auwerx J. Interdependence of AMPK and SIRT1 for metabolic adaptation to fasting and exercise in skeletal muscle. Cell Metab 11: 213-219, 2010.

8. Carafa V, Nebbioso A and Altucci L. Sirtuins and disease: the road ahead. Front Pharmacol 3: 4, 2012.

9. Charge SB and Rudnicki MA. Cellular and molecular regulation of muscle regeneration. Physiol Rev 84: 209-238, 2004.

10. Chen KH, Cheng ML, Jing YH, Chiu DT, Shiao MS and Chen JK. Resveratrol ameliorates metabolic disorders and muscle wasting in streptozotocin-induced diabetic rats. Am J Physiol Endocrinol Metab 301: E853-E863, 2011.

11. Chen SE, Gerken E, Zhang Y, Zhan M, Mohan RK, Li AS, Reid MB and Li YP. Role of TNF-\{alpha\} signaling in regeneration of cardiotoxin-injured muscle. Am J Physiol Cell Physiol 289: C1179-C1187, 2005.

12. d'Albis A, Couteaux R, Janmot C, Roulet A and Mira JC. Regeneration after cardiotoxin injury of innervated and denervated slow and fast muscles of mammals. Myosin isoform analysis. Eur J Biochem 174: 103-110, 1988.

\section{Dolinsky VW, Jones KE, Sidhu RS, Haykowsky M, Czubryt MP, Gordon T and Dyck JR.}

Improvements in skeletal muscle strength and cardiac function induced by resveratrol during exercise training contribute to enhanced exercise performance in rats. J Physiol 590: 2783-2799, 2012. 
14. Fulco M, Schiltz RL, lezzi S, King MT, Zhao P, Kashiwaya $Y$, Hoffman E, Veech RL and Sartorelli V. Sir2 regulates skeletal muscle differentiation as a potential sensor of the redox state. Mol Cell 12: 5162, 2003.

\section{Gerhart-Hines Z, Rodgers JT, Bare O, Lerin C, Kim SH, Mostoslavsky R, Alt FW, Wu Z and}

Puigserver P. Metabolic control of muscle mitochondrial function and fatty acid oxidation through SIRT1/PGC-1alpha. EMBO J 26: 1913-1923, 2007.

16. Harris JB and Johnson MA. Further observations on the pathological responses of rat skeletal muscle to toxins isolated from the venom of the Australian tiger snake, Notechis scutatus scutatus. Clin Exp Pharmacol Physiol 5: 587-600, 1978.

17. Herranz D and Serrano M. SIRT1: recent lessons from mouse models. Nat Rev Cancer 10: 819823, 2010.

18. Hori YS, Kuno A, Hosoda R, Tanno M, Miura T, Shimamoto K and Horio Y. Resveratrol ameliorates muscular pathology in the dystrophic mdx mouse, a model for Duchenne muscular dystrophy. J Pharmacol Exp Ther 338: 784-794, 2011.

19. Hunter RB and Kandarian SC. Disruption of either the $\mathrm{Nfkb} 1$ or the $\mathrm{Bcl} 3$ gene inhibits skeletal muscle atrophy. J Clin Invest 114: 1504-1511, 2004.

20. Jackson JR, Ryan MJ and Alway SE. Long-term supplementation with resveratrol alleviates oxidative stress but does not attenuate sarcopenia in aged mice. J Gerontol A Biol Sci Med Sci 66: 751764, 2011. 
21. Jackson JR, Ryan MJ, Hao Y and Alway SE. Mediation of endogenous antioxidant enzymes and apoptotic signaling by resveratrol following muscle disuse in the gastrocnemius muscles of young and old rats. Am J Physiol Regul Integr Comp Physiol 299: R1572-R1581, 2010.

22. Kaeberlein $\mathbf{M}, \mathbf{M c V}$ ey $\mathbf{M}$ and Guarente $\mathrm{L}$. The SIR2/3/4 complex and SIR2 alone promote longevity in Saccharomyces cerevisiae by two different mechanisms. Genes Dev 13: 2570-2580, 1999.

23. Lagouge M, Argmann C, Gerhart-Hines Z, Meziane H, Lerin C, Daussin F, Messadeq N, Milne J, Lambert P, Elliott P, Geny B, Laakso M, Puigserver P and Auwerx J. Resveratrol improves mitochondrial function and protects against metabolic disease by activating SIRT1 and PGC-1alpha. Cell 127: 1109$1122,2006$.

24. Li H, Rajendran GK, Liu N, Ware C, Rubin BP and Gu Y. SirT1 modulates the estrogen-insulin-like growth factor-1 signaling for postnatal development of mammary gland in mice. Breast Cancer Res 9: R1, 2007.

25. Lin J, Wu H, Tarr PT, Zhang CY, Wu Z, Boss O, Michael LF, Puigserver P, Isotani E, Olson EN, Lowell BB, Bassel-Duby R and Spiegelman BM. Transcriptional co-activator PGC-1 alpha drives the formation of slow-twitch muscle fibres. Nature 418: 797-801, 2002.

26. Momken I, Stevens L, Bergouignan A, Desplanches D, Rudwill F, Chery I, Zahariev A, Zahn S, Stein TP, Sebedio JL, Pujos-Guillot E, Falempin M, Simon C, Coxam V, Andrianjafiniony T, GauquelinKoch G, Picquet F and Blanc S. Resveratrol prevents the wasting disorders of mechanical unloading by acting as a physical exercise mimetic in the rat. FASEB J 25: 3646-3660, 2011. 
27. Mouisel E, Vignaud A, Hourde C, Butler-Browne G and Ferry A. Muscle weakness and atrophy are associated with decreased regenerative capacity and changes in mTOR signaling in skeletal muscles of venerable (18-24-month-old) dystrophic mdx mice. Muscle Nerve 41: 809-818, 2010.

28. Mourkioti F, Kratsios P, Luedde T, Song YH, Delafontaine P, Adami R, Parente V, Bottinelli R, Pasparakis $\mathbf{M}$ and Rosenthal $\mathbf{N}$. Targeted ablation of IKK2 improves skeletal muscle strength, maintains mass, and promotes regeneration. J Clin Invest 116: 2945-2954, 2006.

29. Rathbone CR, Booth FW and Lees SJ. Sirt1 increases skeletal muscle precursor cell proliferation. Eur J Cell Biol 88: 35-44, 2009.

30. Rogina B and Helfand SL. Sir2 mediates longevity in the fly through a pathway related to calorie restriction. Proc Natl Acad Sci U S A 101: 15998-16003, 2004.

31. Sadoul K, Boyault C, Pabion M and Khochbin S. Regulation of protein turnover by acetyltransferases and deacetylases. Biochimie 90: 306-312, 2008.

32. Salminen A and Kaarniranta K. NF-kappaB signaling in the aging process. J Clin Immunol 29: 397405, 2009.

33. Sandri M, Lin J, Handschin C, Yang W, Arany ZP, Lecker SH, Goldberg AL and Spiegelman BM. PGC-1alpha protects skeletal muscle from atrophy by suppressing FoxO3 action and atrophy-specific gene transcription. Proc Natl Acad Sci U S A 103: 16260-16265, 2006. 
34. Selsby JT, Morine KJ, Pendrak K, Barton ER and Sweeney HL. Rescue of dystrophic skeletal muscle by PGC-1alpha involves a fast to slow fiber type shift in the mdx mouse. PLoS One 7: e30063, 2012.

35. Shadfar S, Couch ME, McKinney KA, Weinstein L, Yin X, Rodriguez JE, Guttridge DC and Willis

M. Oral resveratrol therapy inhibits cancer-induced skeletal muscle and cardiac atrophy in vivo. Nutr Cancer 63: 749-762, 2011.

36. Siu PM, Pistilli EE, Butler DC and Alway SE. Aging influences cellular and molecular responses of apoptosis to skeletal muscle unloading. Am J Physiol Cell Physiol 288: C338-C349, 2005.

37. Tissenbaum HA and Guarente L. Increased dosage of a sir-2 gene extends lifespan in Caenorhabditis elegans. Nature 410: 227-230, 2001.

38. Tonkin J, Villarroya F, Puri PL and Vinciguerra M. SIRT1 signaling as potential modulator of skeletal muscle diseases. Curr Opin Pharmacol 2012.

39. Van Gammeren D, Damrauer JS, Jackman RW and Kandarian SC. The IkappaB kinases IKKalpha and IKKbeta are necessary and sufficient for skeletal muscle atrophy. FASEB J 23: 362-370, 2009.

40. Wenz T, Rossi SG, Rotundo RL, Spiegelman BM and Moraes CT. Increased muscle PGC-1alpha expression protects from sarcopenia and metabolic disease during aging. Proc Natl Acad Sci U S A 106: 20405-20410, 2009. 
41. Yeung F, Hoberg JE, Ramsey CS, Keller MD, Jones DR, Frye RA and Mayo MW. Modulation of NF-kappaB-dependent transcription and cell survival by the SIRT1 deacetylase. EMBO J 23: 2369-2380, 2004. 


\section{Tables}

Table 1

\begin{tabular}{|c|c|c|c|c|c|c|}
\hline & & Day 3 & & & Day7 & \\
\hline Genotype & Bodyweight (g) & Control TA $(\mathrm{mg} / \mathrm{g})$ & Injured $\mathrm{TA}(\mathrm{mg} / \mathrm{g})$ & Bodyweight (g) & Control TA $|\mathrm{mg} / \mathrm{g}|$ & Injured TA $\mid \mathrm{mg} / \mathrm{g})$ \\
\hline Sirt1 $* W T$ & $34.97 \pm 2.62$ & $1.86 \pm .14$ & $1.15 \pm .20$ & $32.53 \pm 1.95$ & $1.90 \pm .20$ & $1.21 \pm .12 \#$ \\
\hline sirt1 + & $36.46 \pm 6.57$ & $1.33 \pm .17 \dagger$ & $.83 \pm .10 \#^{*}$ & $31.40 \pm 4.13$ & $1.37 \pm .13 \dagger$ & $.83 \pm .10 \mathrm{H}^{*}$ \\
\hline Sirt1 $+{ }^{++}$WT & $45.23 \pm 9.88$ & $1.28 \pm .14$ & $.82 \pm .15 \#$ & $38.47 \pm 9.18$ & $1,30 \pm .16$ & $.79 \pm .23 \#$ \\
\hline Sirt1 $1^{++}$ & $45.98 \pm 14.25$ & $1.32 \pm .29$ & $.85 \pm .21 \#$ & $45.23 \pm 5.32$ & $1.35 \pm .17$ & $.94 \pm .17 \#$ \\
\hline
\end{tabular}




\section{Figures}

Figure 1A

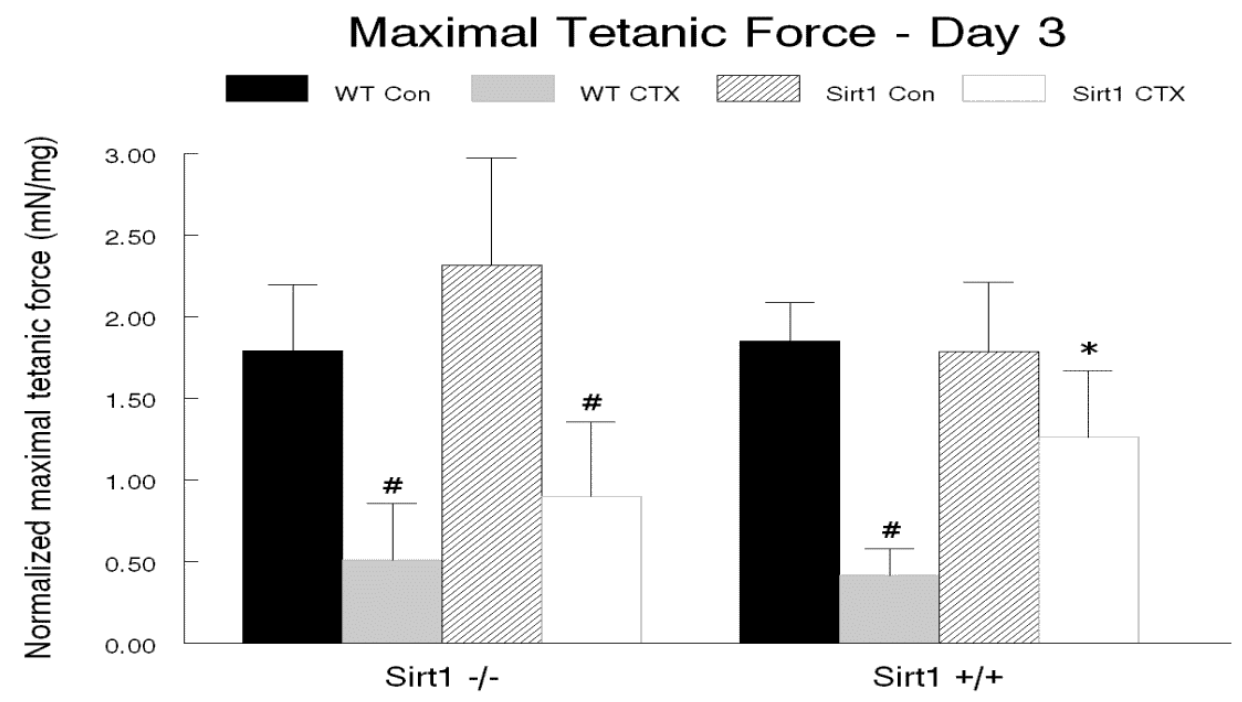

Figure 1B

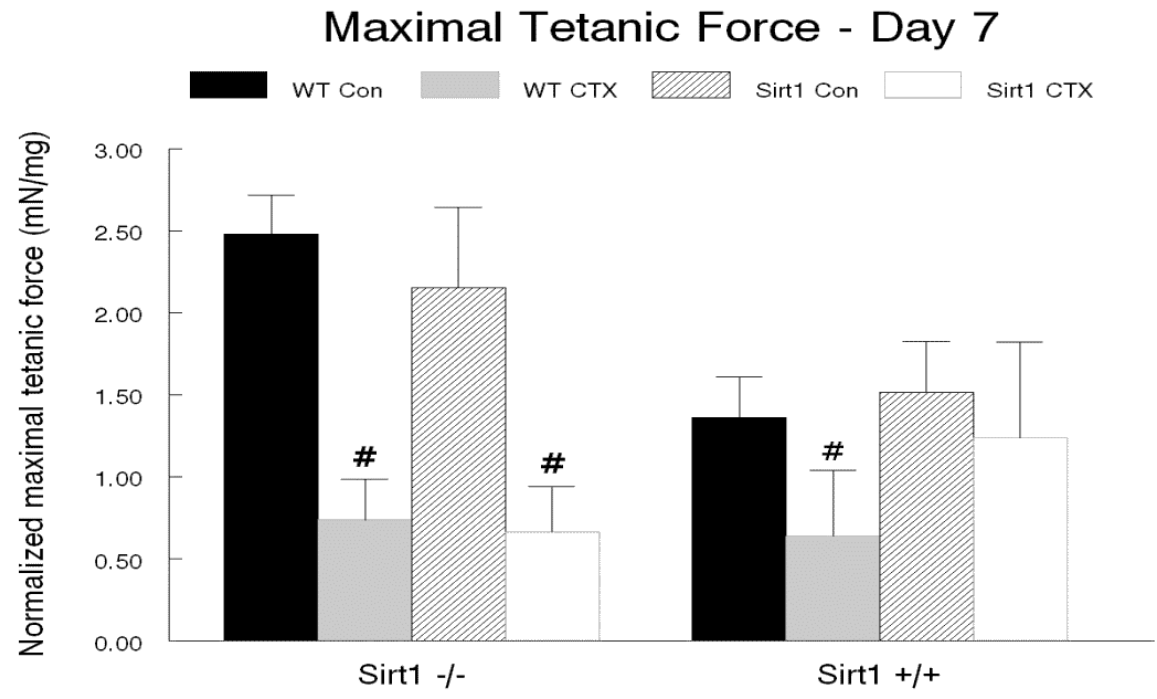


Figure 2A

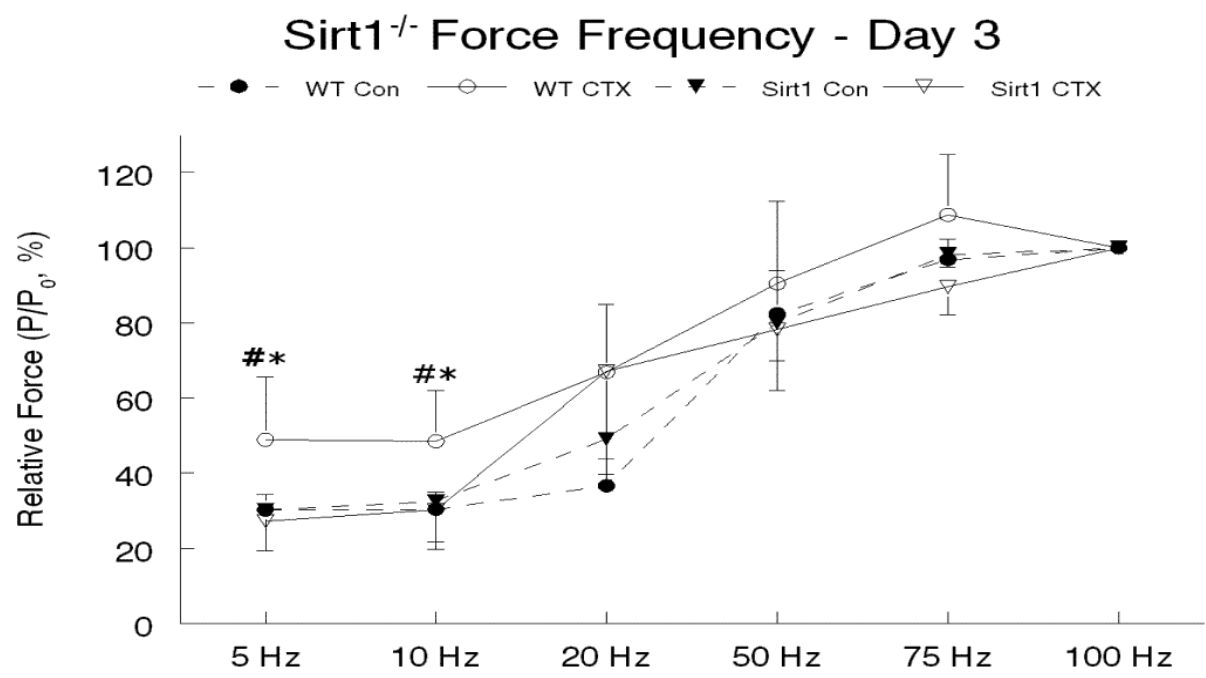

Figure 2B

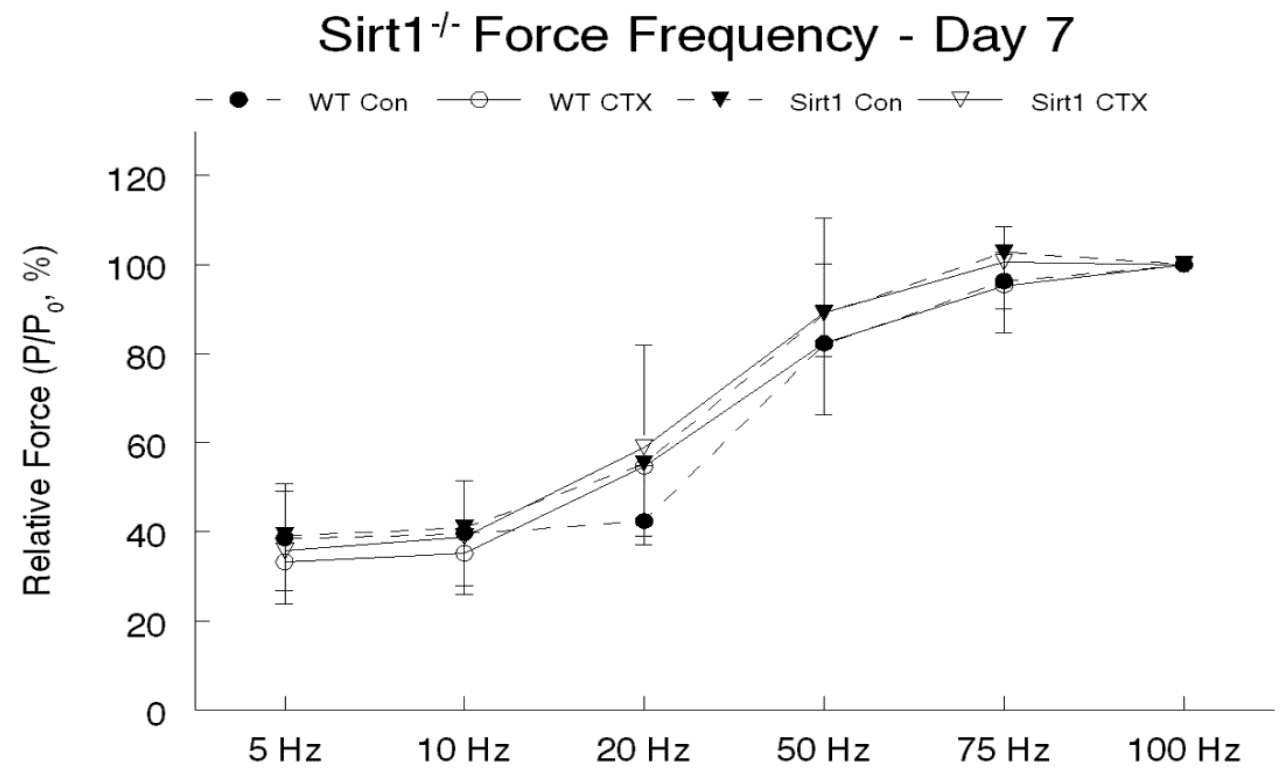


Figure 2C

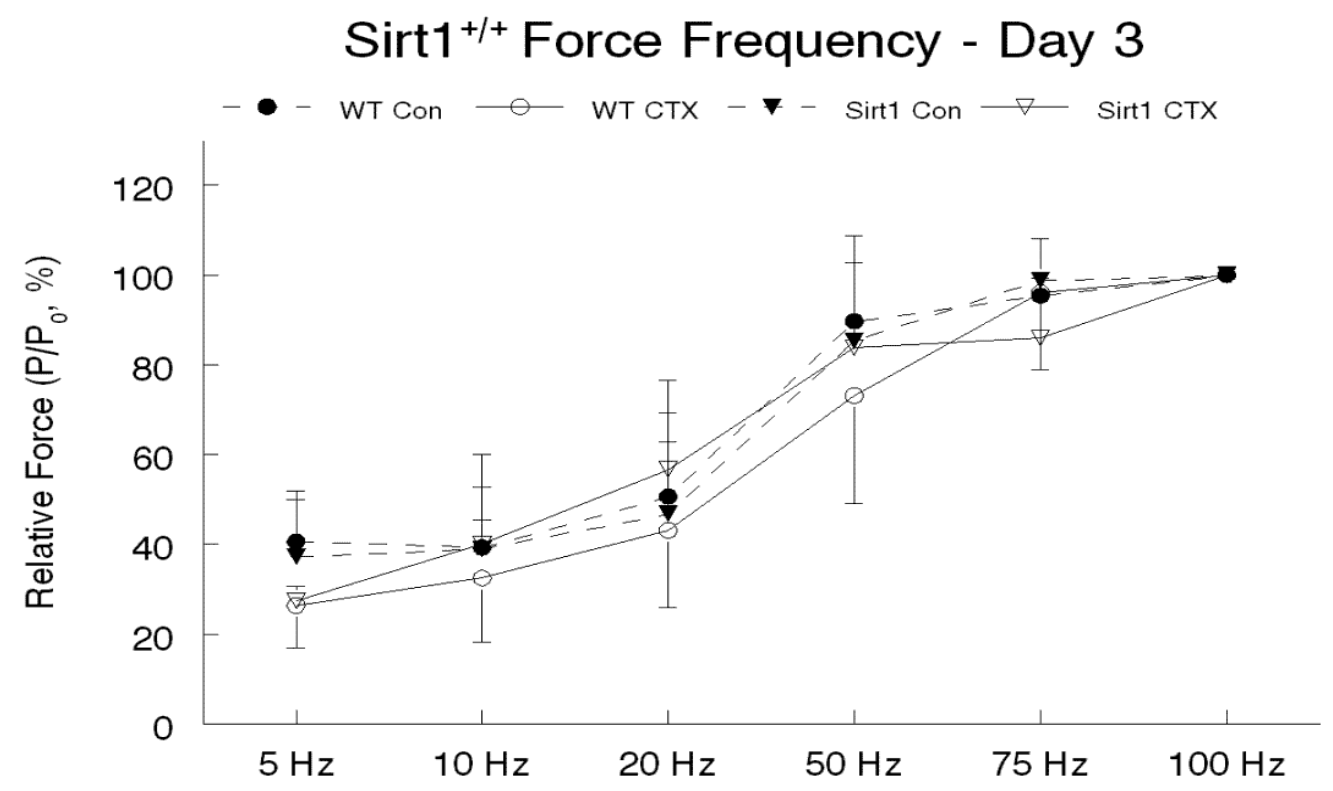

Figure 2D

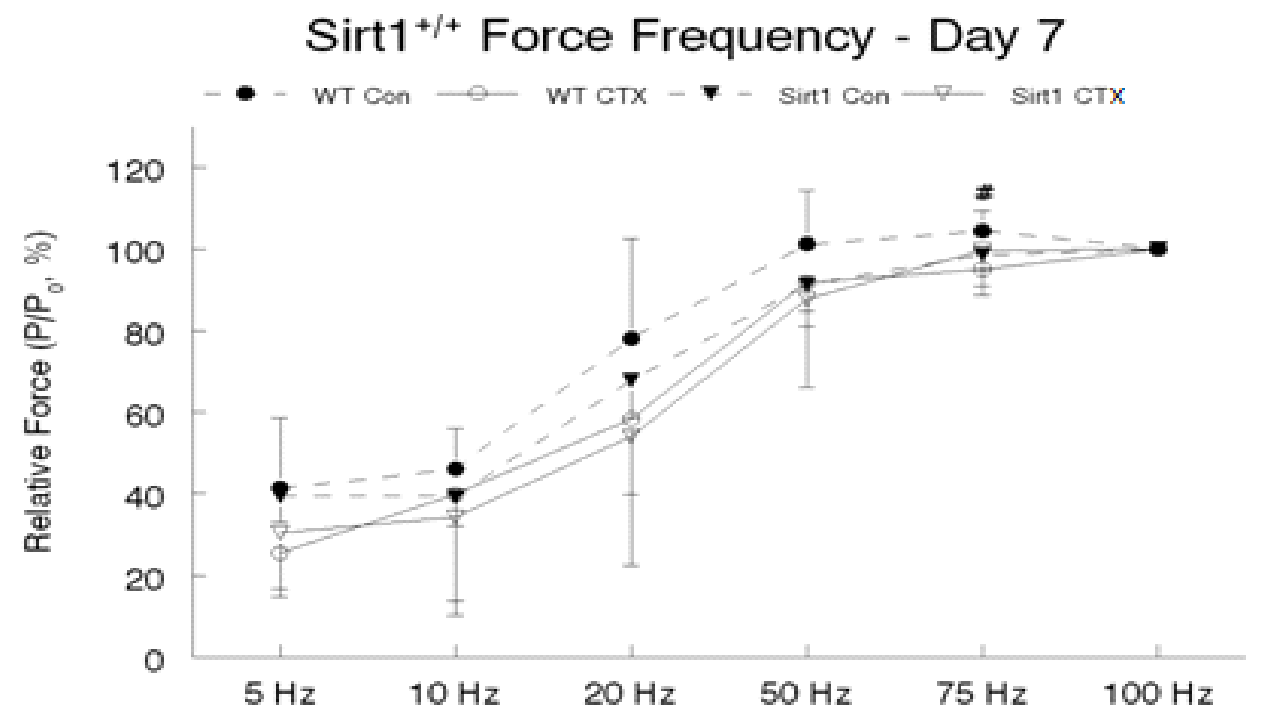


Figure 3A

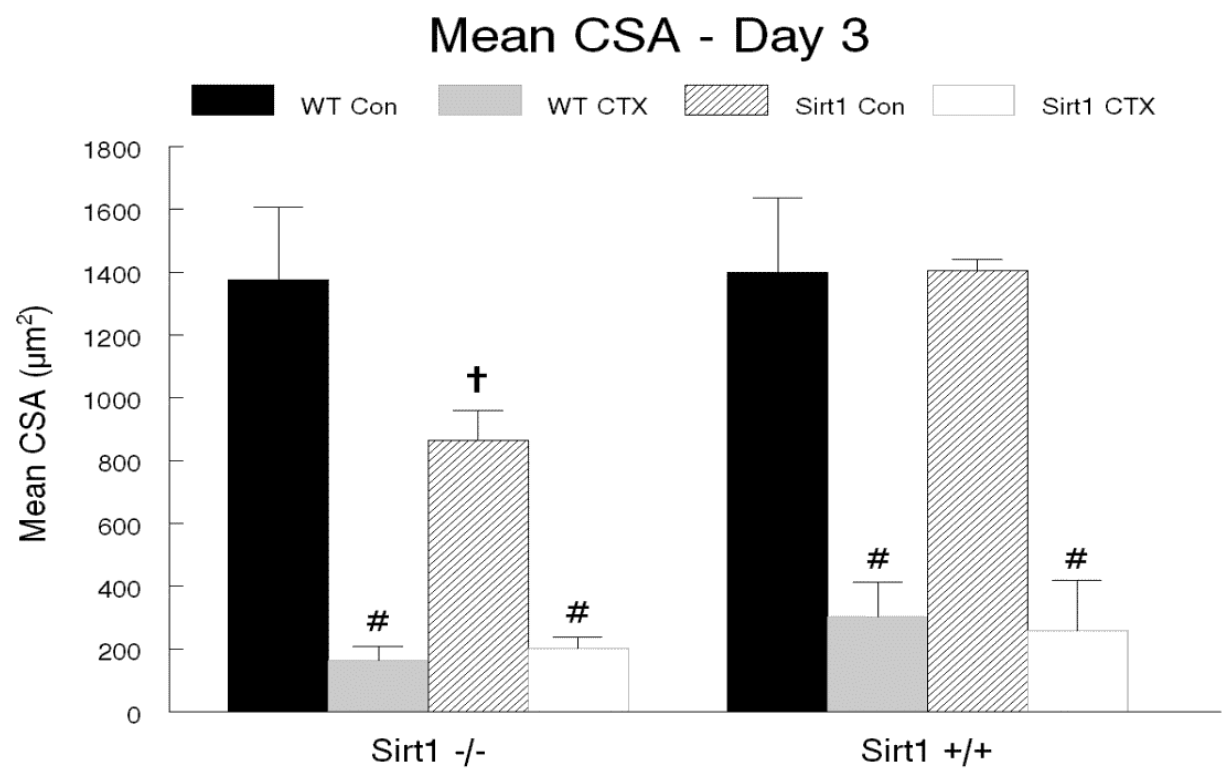

Figure 3B

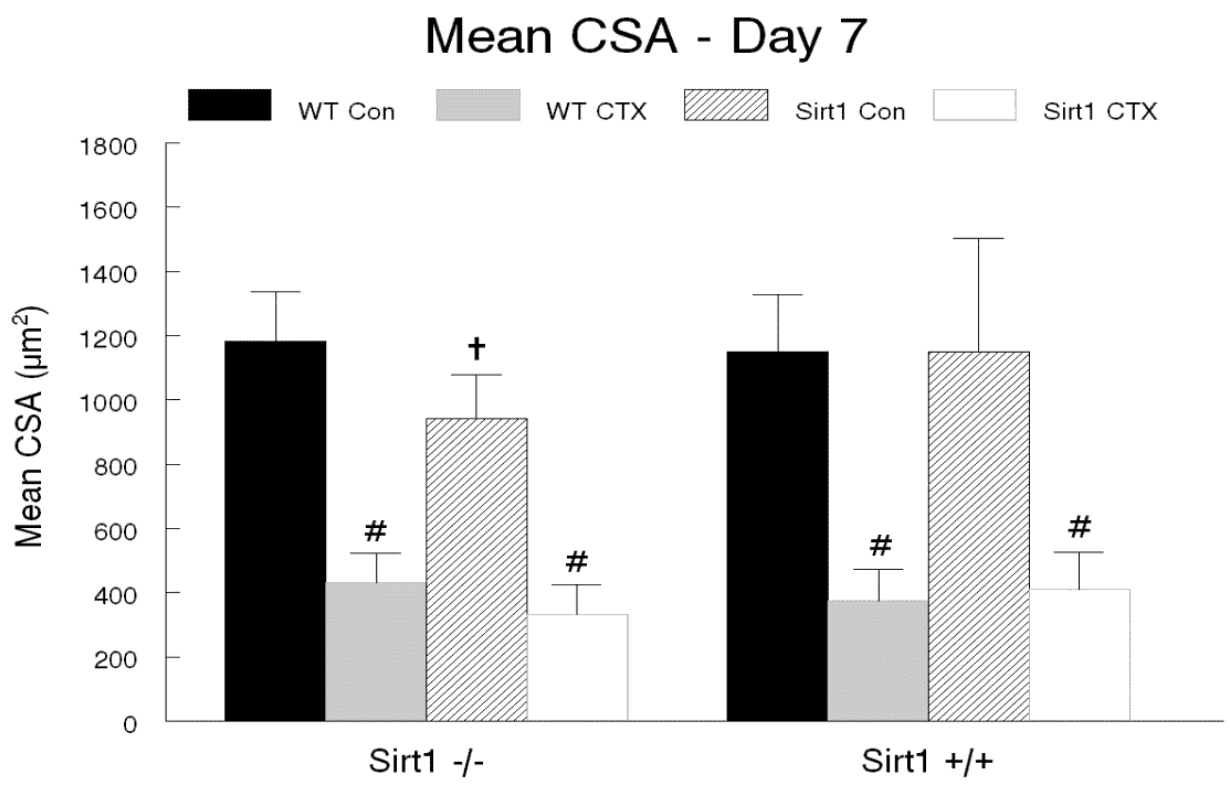


Figure 3C

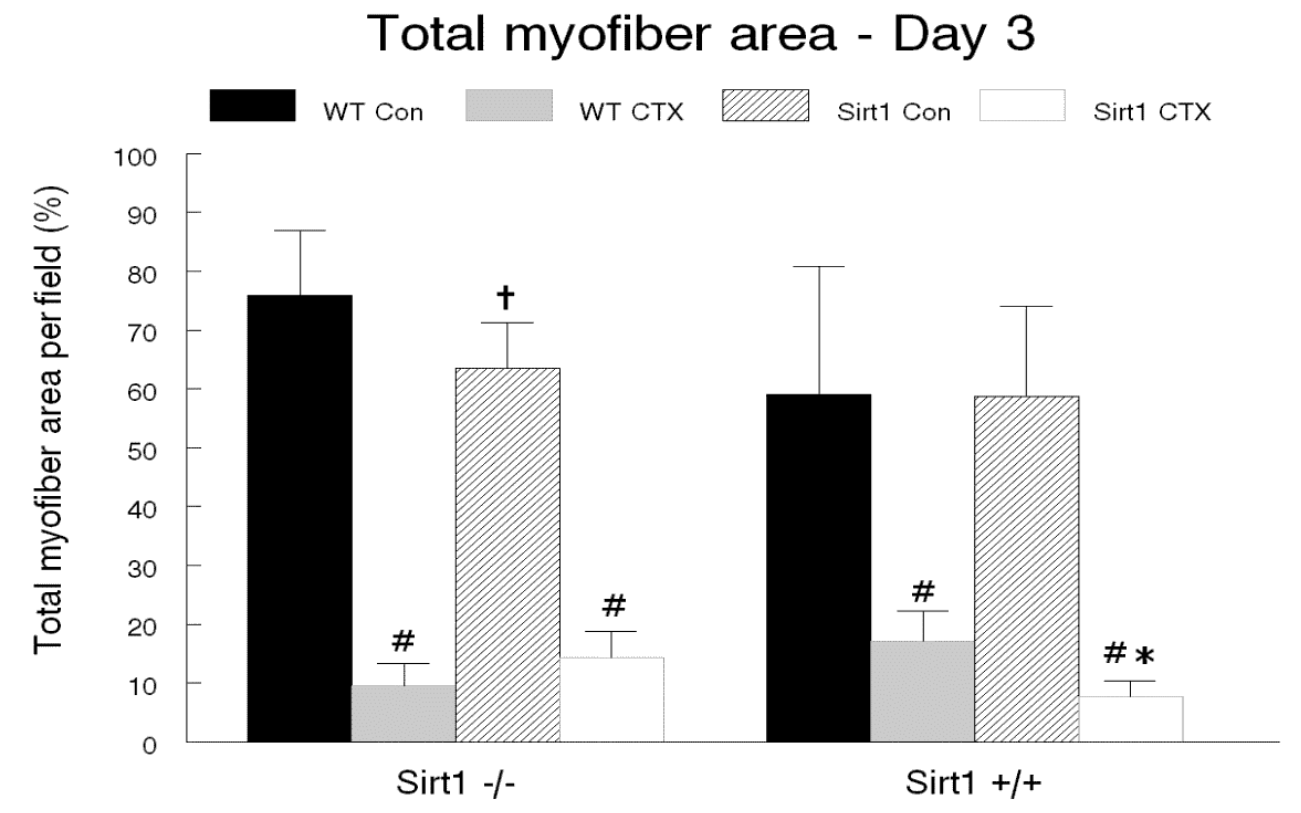

Figure 3D

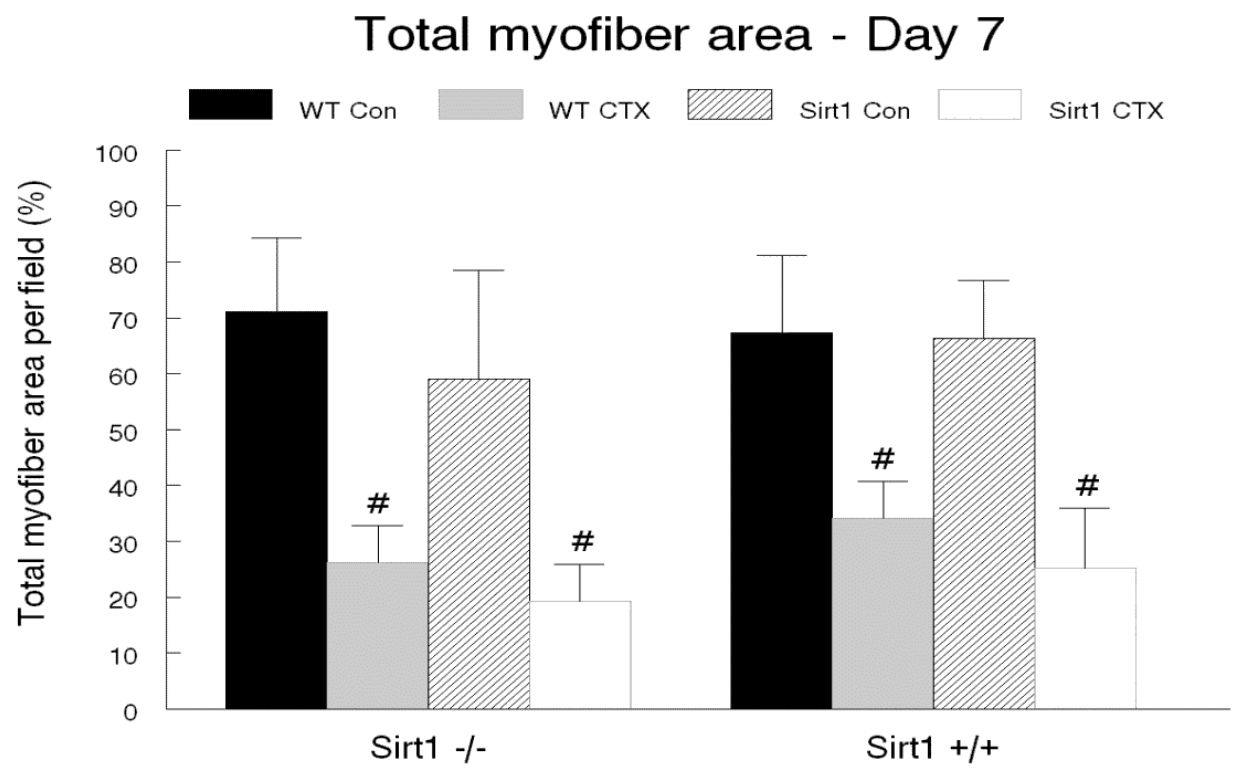


Figure 4A

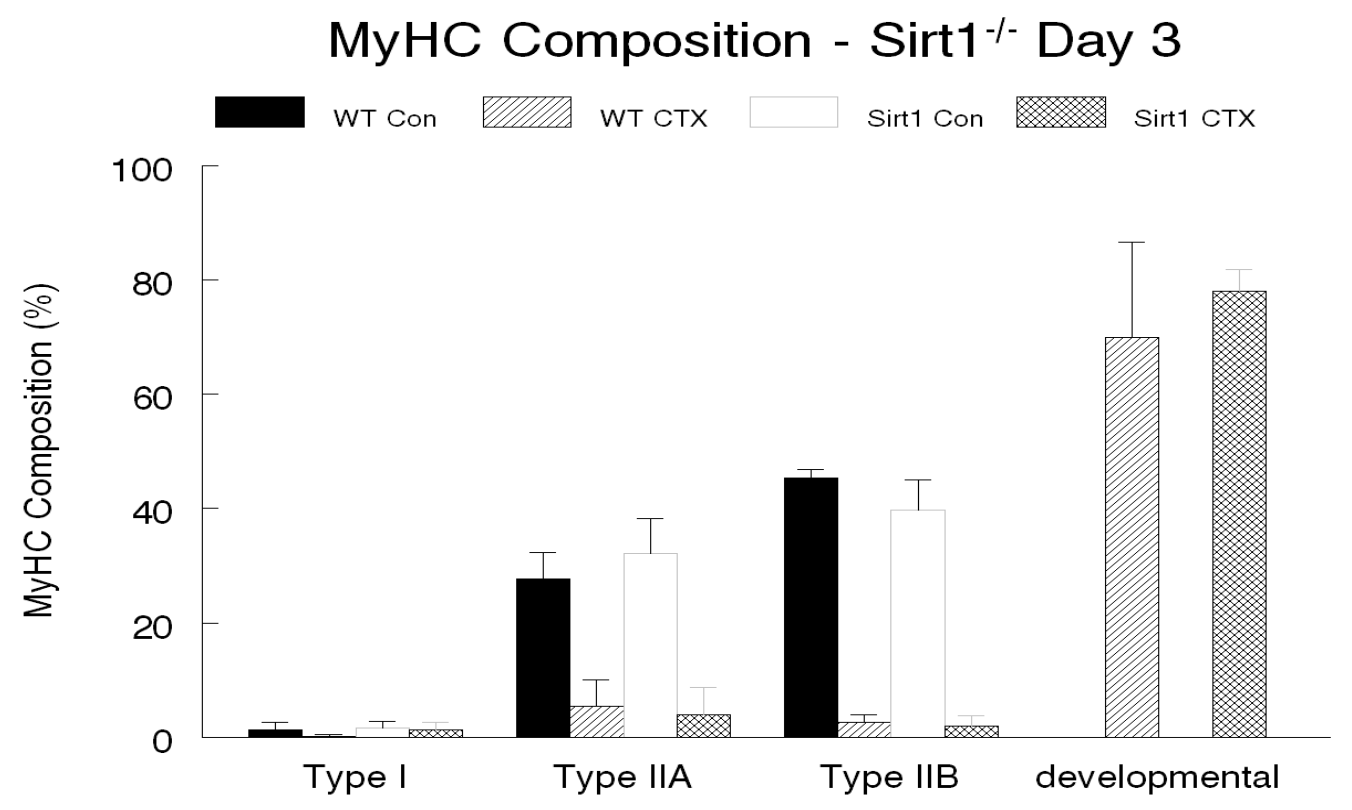

Figure 4B

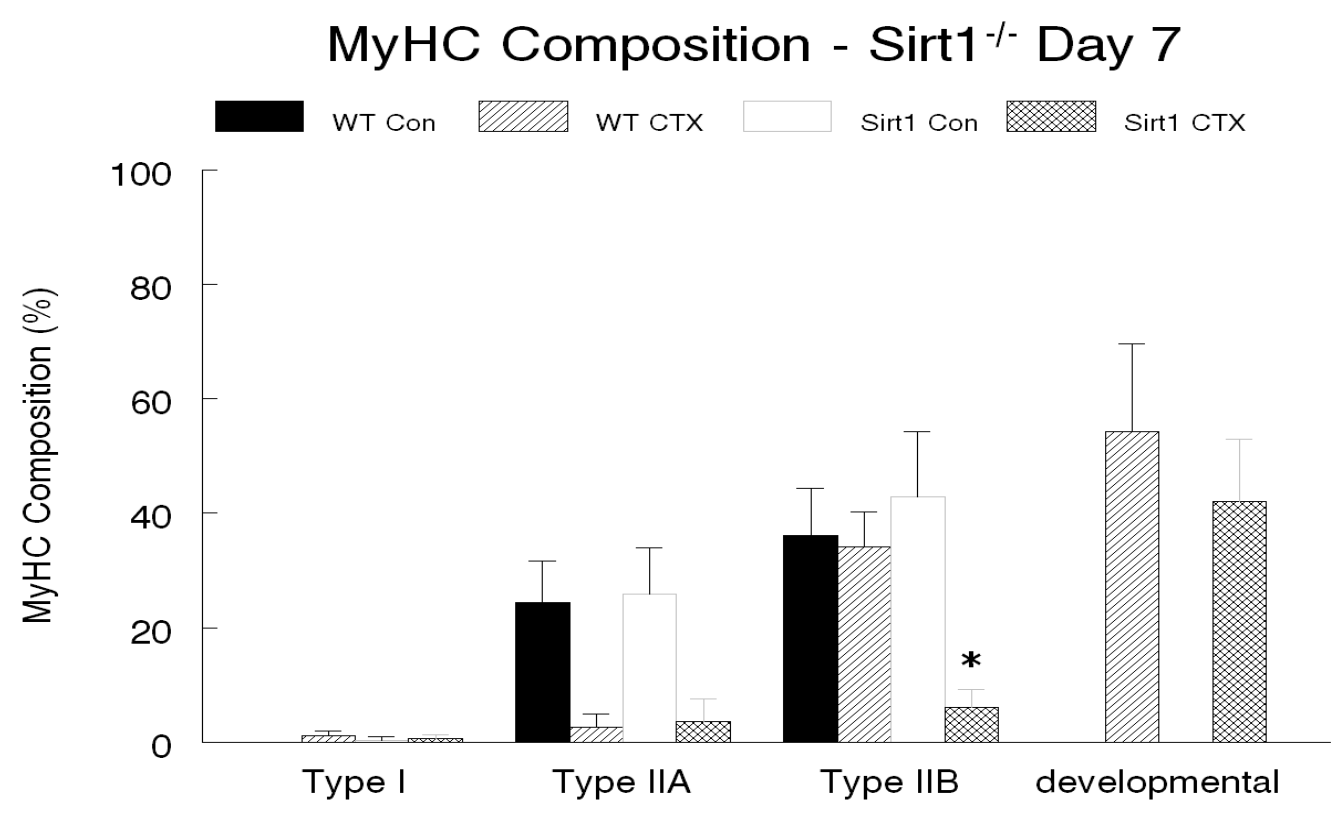


Figure 4C

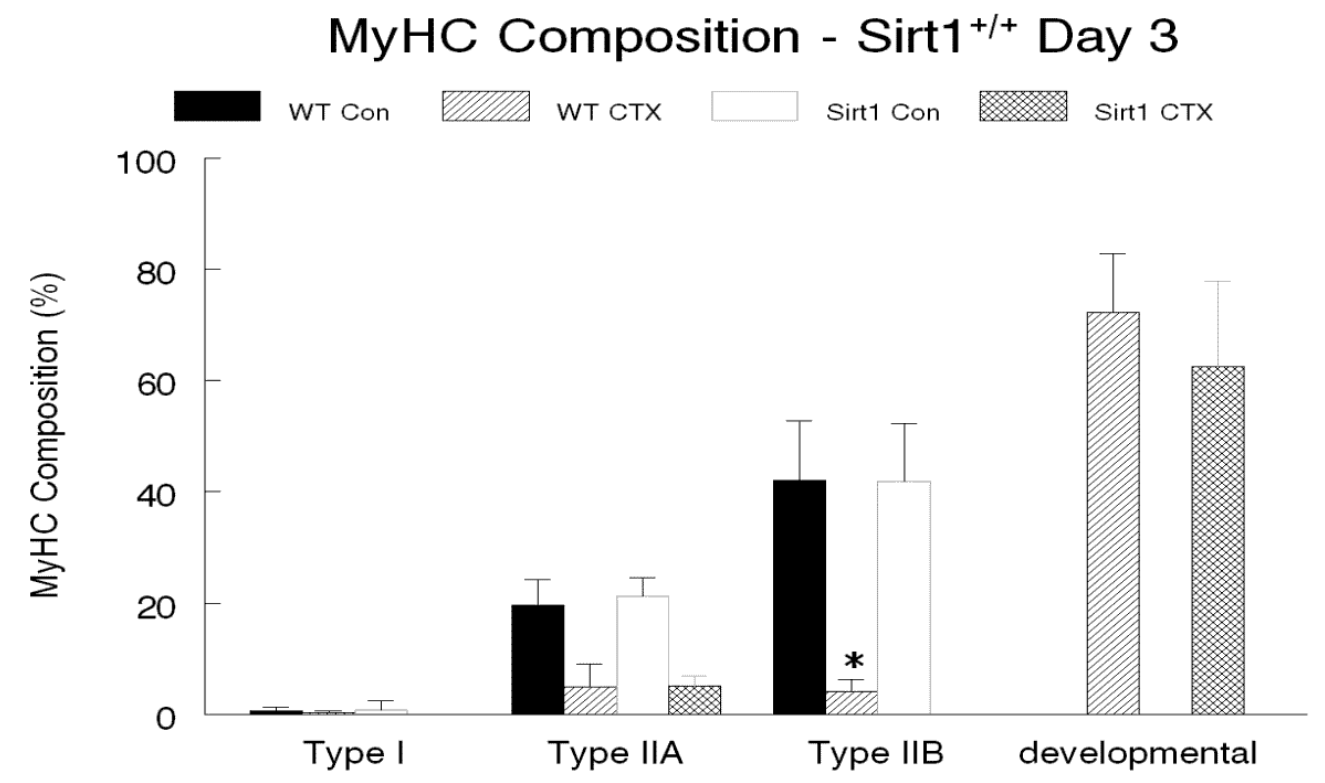

Figure 4D

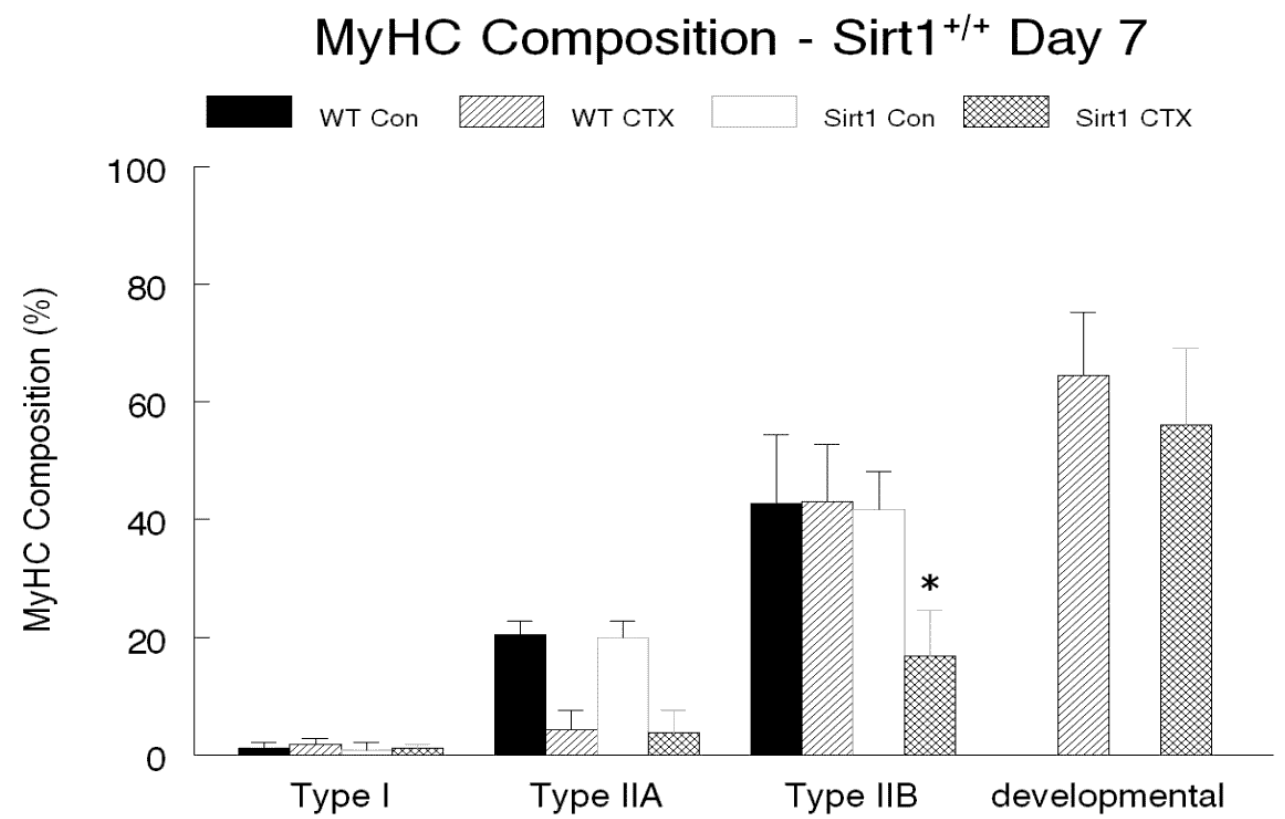


Figure 5A

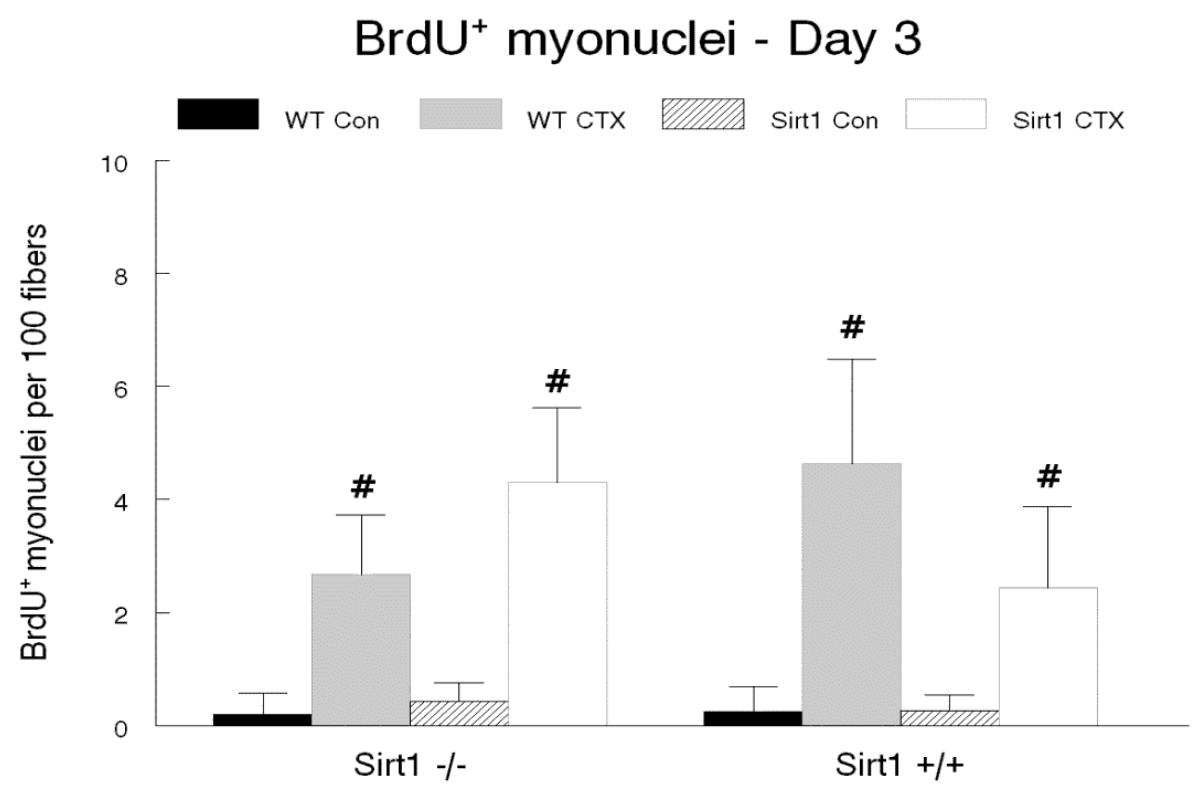

Figure 5B

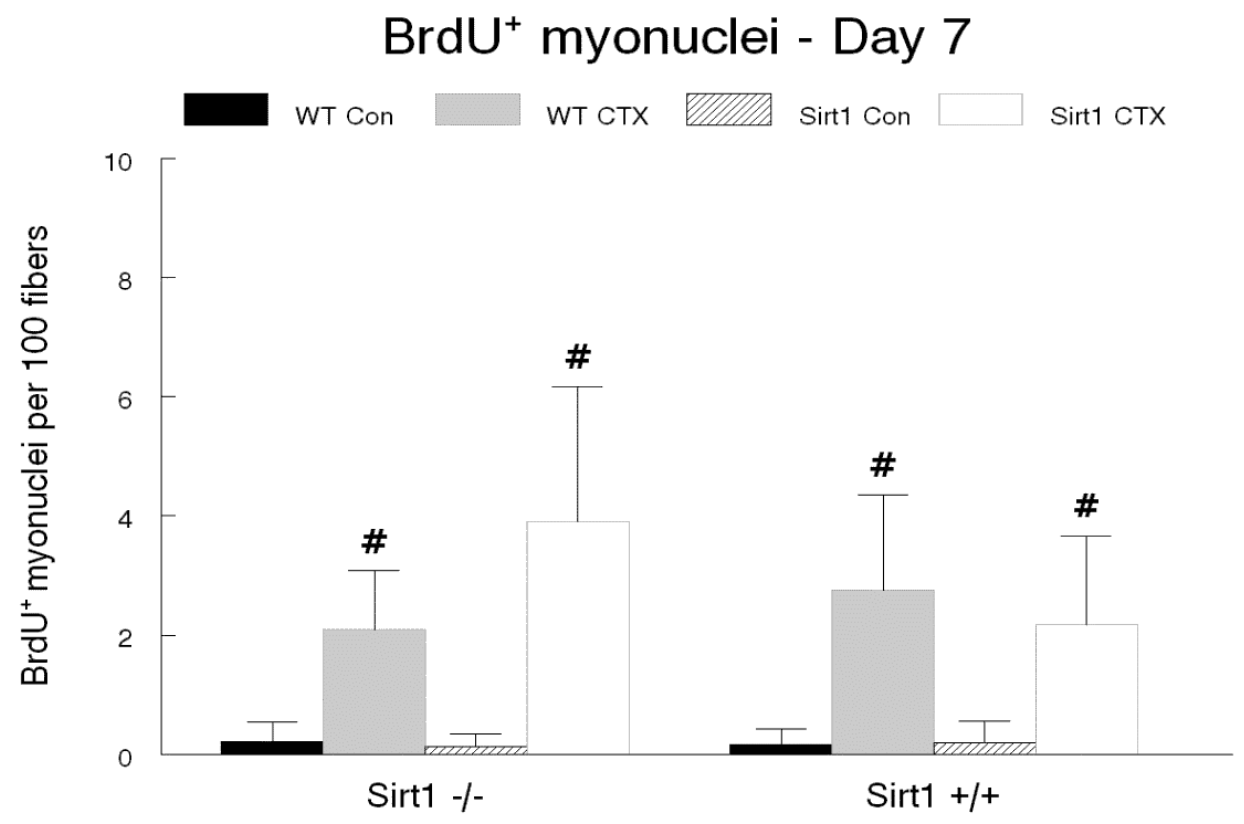


Figure 6A

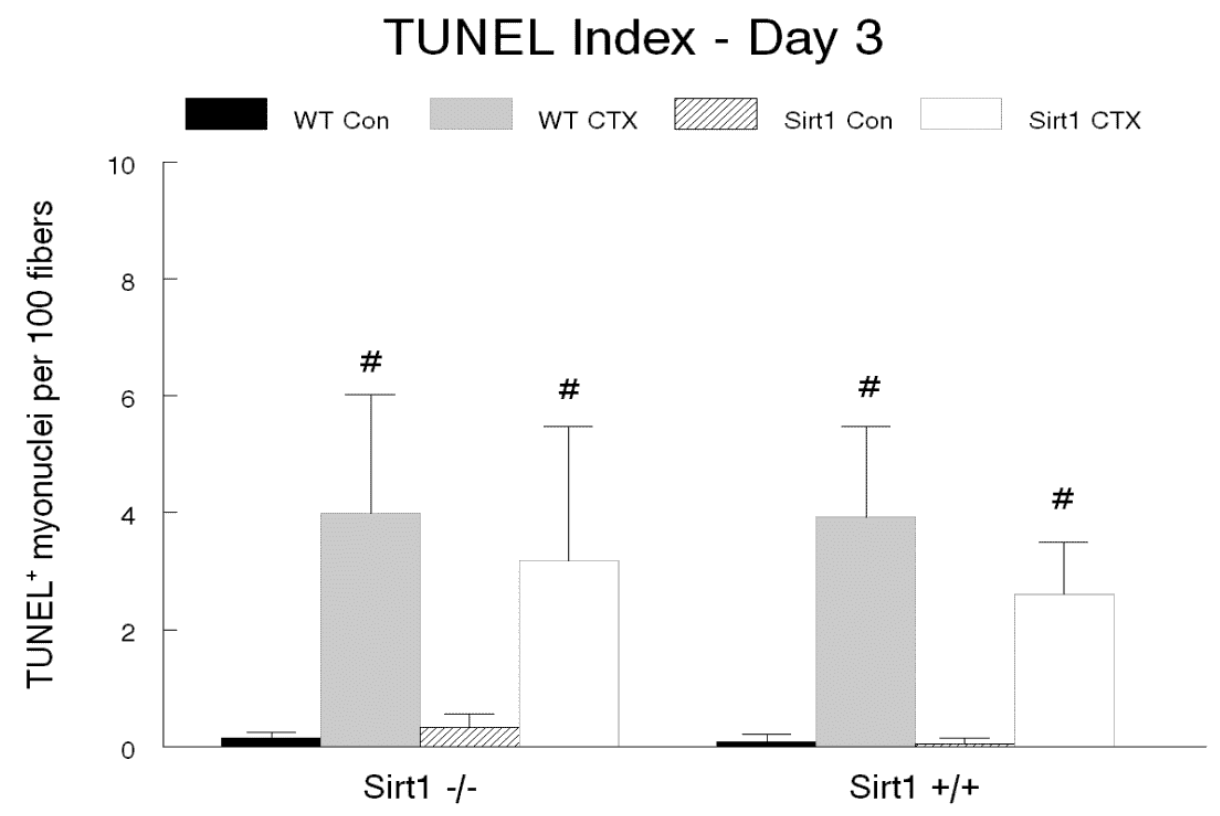

Figure 6B

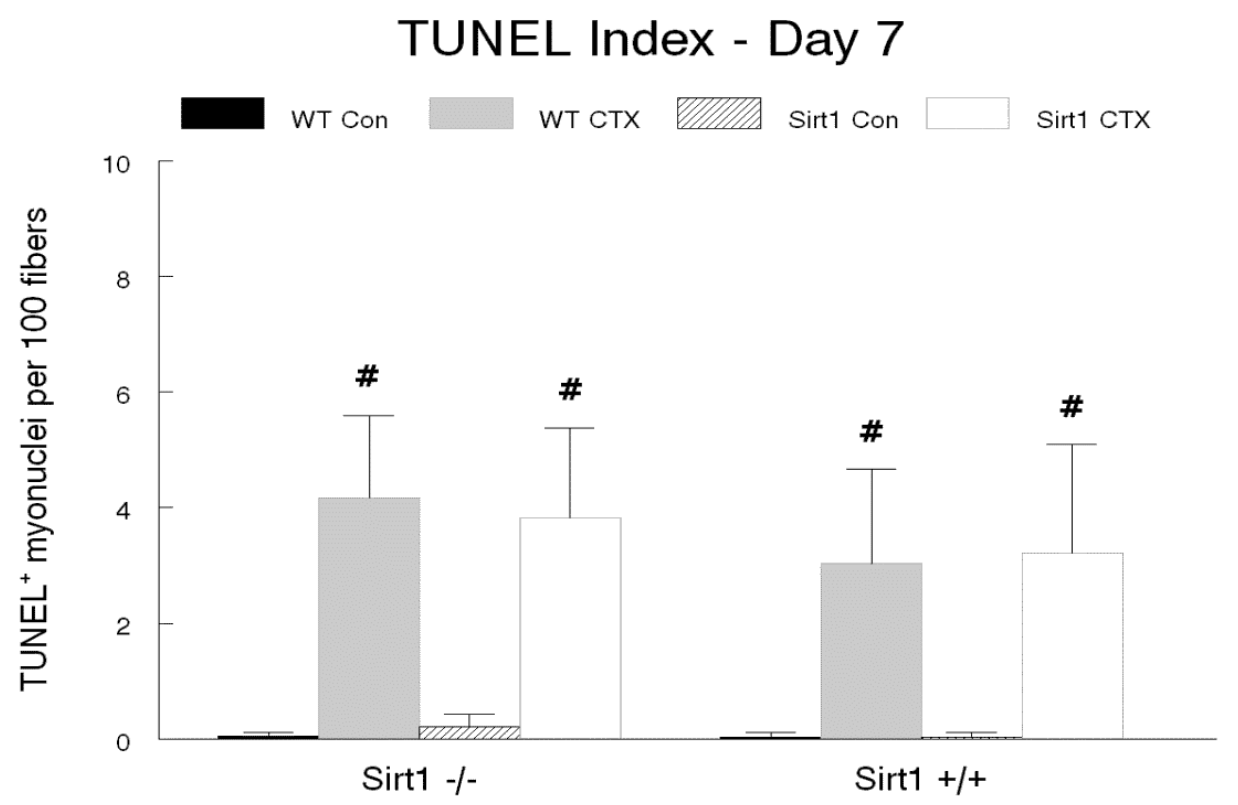




\section{Chapter 5}

\section{Discussion}

The main objective of this thesis project was to examine the role of Sirt1 in skeletal muscle regeneration. For this, we included two specific aims and each required a separate study to address the hypotheses. We employed both nutritional and genetic techniques in an attempt to understand the effects of Sirt1 modulation on the regeneration of skeletal muscle in response to a perturbation in homeostasis. Our hope was to advance the field of muscle regeneration and provide novel avenues to address this issue.

\section{Specific aim 1}

Our first specific aim utilized resveratrol, a known Sirt1 activator, as a nutritional supplement in an attempt to improve muscle regeneration in aged rats following a period of disuse. We had hypothesized that resveratrol would improve regeneration (morphological and/or functional characteristics) in these animals by reducing mitochondrial-associated apoptosis, preserving muscle mass, and increasing the activation of satellite cells during a period of reloading which immediately followed disuse.

The results from this study provided evidence that resveratrol may provide a modest benefit in regenerating muscles of aged animals. Although it proved ineffective at attenuating bodyweight and muscle mass decreases due to mechanical unloading, resveratrol was able to provide partial protection from the accompanying decrease in myofiber area in type II MyHC fibers. Moreover, resveratrol did not statistically increase the activation of satellite cells as indicated by a similar quantity of BrdU-labeled myonuclei during the reloading period in vehicle and resveratrol treated animals. Nevertheless, resveratrol increased the relative protein abundance of the anti-apoptotic Bcl-xL protein during the suspension period, which may have contributed to the slight protection against myofiber area reductions observed in these animals. 
Therefore, the attenuation of myofiber area loss during $\mathrm{HS}$ and reloading combined with the beneficial effects on apoptotic signaling lead us to conclude that resveratrol supplementation may be useful as a potential therapy to counteract muscle loss and improve regeneration in aged muscles in response to extended periods of disuse.

One obvious aspect of this study that could be manipulated in future research is the dose of resveratrol utilized. In our study, we used $125 \mathrm{mg} / \mathrm{kg} /$ day, and this dose provided subtle benefits during unloading and reloading of skeletal muscle. However, this dose could be increased substantially in order to determine if this will maximize the potential benefits of this supplement. It is possible that by increasing the dose of resveratrol, satellite cells would be activated to a larger degree, thereby improving muscle size and possibly function. Therefore, future research should attempt to determine the optimal dose of resveratrol to maximize its beneficial effects. Moreover, although our current study was specifically conducted to determine resveratrol's effectiveness in aged animals, future studies could utilize younger animals in order to maximize any potential responses, as these animals have much higher regenerative abilities.

\section{Specific aim 2}

The main objective of the second aim was to directly examine the role of Sirt1 expression on muscle regeneration in older-age adult mice. In order to accomplish this, two separate transgenic lines of mice were utilized which presented either the knockdown of Sirt1 expression $\left(\operatorname{Sirt}^{-/-}\right)$or the overexpression of Sirt1 $\left(\operatorname{Sirt} 1^{+/+}\right)$. We injured the animal's muscles with an acute myotoxin injection and subsequently analyzed the muscle regeneration characteristics at both 3 and 7 days post-injury.

Overexpression of Sirt1 did not improve muscle mass or fiber area in regenerating muscles at either 3 or 7 days, nor was it detrimental for these measures when Sirt1 expression was decreased in the Sirt1 ${ }^{-/}$animals. However, it was determined that increasing Sirt1 
expression was beneficial to muscle force. Following muscle injury, the maximal tetanic force produced in the muscles of the Sirt $1^{+/+}$animals was no different than that of the uninjured control muscles at both 3 and 7 days post-injury. This is in contrast to the Sirt $1^{+/+}$WT animals, whose maximal tetanic force still had not recovered by day 7 post-injury. Thus, we concluded from this study that increasing Sirt1 may be beneficial to muscle regeneration in that it improved muscle function (i.e., maximal tetanic force) which is very important in various clinical situations.

One possible explanation for the lack of increase in activation of satellite cells in Sirt $1^{+/+}$ animals is that the rate of satellite cell activation was already at the maximal rate in the CTX model of severe damage and recovery. If that was the case, then additional intervention could not have increased the activation of satellite cells. To test this hypothesis, we would require an approach that would titrate the injury stimulus (e.g., stretch induced injury), and then evaluate if Sirt $1^{+/+}$mice had a greater activation of satellite cells in a less severe injury.

As this study examined muscle regeneration in an almost exclusively fast muscle (tibialis anterior), future research should be directed at examining the role of Sirt1 in muscle regeneration in other muscle types such as the slow-twitch soleus muscle, in order to establish whether Sirt1 may be more beneficial in certain fiber types. Thus, it is possible that this study could be repeated in the future, but with the injury being directed towards a different muscle with alternate fiber types. This may lead to therapies utilizing Sirt1 that are specialized for varying clinical conditions.

\section{A look to the future}

Herein we provide support for the beneficial role of Sirt1 in skeletal muscle regeneration. Undertaking two separate studies with various muscle perturbations to address our specific aims, our hypotheses from each specific aim were confirmed as Sirt1 attenuated decreases in myofiber area in response to disuse and improved muscle force during the early stages of regeneration. While far from complete, our research provides a positive base for future 
research to examine the role and potential benefits of Sirt1 in varying clinical conditions as a novel therapeutic intervention. Many diseases and conditions require some form of skeletal muscle maintenance and regeneration and therapies must be targeted to individualized circumstances. Although our research did not yield any astonishing results to revolutionize the field, we have provided sufficient evidence to warrant future research into the study of the utilization of Sirt1 as a therapeutic to improve skeletal muscle regeneration in various conditions. Only time may reveal the full impact of these findings and the extent to which they may benefit those in need. 
1. Abraham IL, Bottrell MM, Dash KR, Fulmer TT, Mezey MD, O'Donnell L and Vince-Whitman C. Profiling care and benchmarking best practice in care of hospitalized elderly: the Geriatric Institutional Assessment Profile. Nurs Clin North Am 34: 237-255, 1999.

2. Adhihetty PJ, O'Leary MF and Hood DA. Mitochondria in skeletal muscle: adaptable rheostats of apoptotic susceptibility. Exerc Sport Sci Rev 36: 116-121, 2008.

3. Alamdari N, Aversa Z, Castillero E, Gurav A, Petkova V, Tizio S and Hasselgren PO. Resveratrol prevents dexamethasone-induced expression of the muscle atrophy-related ubiquitin ligases atrogin-1 and MuRF1 in cultured myotubes through a SIRT1-dependent mechanism. Biochem Biophys Res Commun 417: 528-533, 2012.

4. Alcendor RR, Gao S, Zhai P, Zablocki D, Holle E, Yu X, Tian B, Wagner T, Vatner SF and Sadoshima J. Sirt1 regulates aging and resistance to oxidative stress in the heart. Circ Res 100: 1512-1521, 2007.

5. Alkner BA and Tesch PA. Efficacy of a gravity-independent resistance exercise device as a countermeasure to muscle atrophy during 29-day bed rest. Acta Physiol Scand 181: 345-357, 2004

6. Allen RE, Sheehan SM, Taylor RG, Kendall TL and Rice GM. Hepatocyte growth factor activates quiescent skeletal muscle satellite cells in vitro. J Cell Physiol 165: 307-312, 1995. 
7. Alway SE. Attenuation of $\mathrm{Ca}(2+)$-activated ATPase and shortening velocity in hypertrophied fast twitch skeletal muscle from aged Japanese quail. Exp Gerontol 37: 665-678, 2002.

8. Andersen JL, Gruschy-Knudsen T, Sandri C, Larsson L and Schiaffino S. Bed rest increases the amount of mismatched fibers in human skeletal muscle. J Appl Physiol 86: 455-460, 1999.

9. Antonsson B, Conti F, Ciavatta A, Montessuit S, Lewis S, Martinou I, Bernasconi L, Bernard A, Mermod JJ, Mazzei G, Maundrell K, Gambale F, Sadoul R and Martinou JC. Inhibition of Bax channel-forming activity by Bcl-2. Science 277: 370-372, 1997.

10. Baliga MS, Meleth S and Katiyar SK. Growth inhibitory and antimetastatic effect of green tea polyphenols on metastasis-specific mouse mammary carcinoma 4T1 cells in vitro and in vivo systems. Clin Cancer Res 11: 1918-1927, 2005.

11. Bamman MM, Clarke MS, Feeback DL, Talmadge RJ, Stevens BR, Lieberman SA and Greenisen MC. Impact of resistance exercise during bed rest on skeletal muscle sarcopenia and myosin isoform distribution. J Appl Physiol 84: 157-163, 1998.

12. Barani AE, Durieux AC, Sabido $\mathbf{O}$ and Freyssenet $\mathbf{D}$. Age-related changes in the mitotic and metabolic characteristics of muscle-derived cells. J Appl Physiol 95: 2089-2098, 2003.

13. Barazzoni R, Short KR and Nair KS. Effects of aging on mitochondrial DNA copy number and cytochrome c oxidase gene expression in rat skeletal muscle, liver, and heart. J Biol Chem 275: 3343-3347, 2000. 
14. Barton ER, Morris L, Musaro A, Rosenthal N and Sweeney HL. Muscle-specific expression of insulin-like growth factor I counters muscle decline in mdx mice. J Cell Biol 157: 137-148, 2002.

15. Baumgartner RN, Koehler KM, Gallagher D, Romero L, Heymsfield SB, Ross RR, Garry PJ and Lindeman RD. Epidemiology of sarcopenia among the elderly in New Mexico. Am J Epidemiol 147: 755-763, 1998.

16. Baur JA, Pearson KJ, Price NL, Jamieson HA, Lerin C, Kalra A, Prabhu VV, Allard JS, Lopez-Lluch G, Lewis K, Pistell PJ, Poosala S, Becker KG, Boss O, Gwinn D, Wang M, Ramaswamy S, Fishbein KW, Spencer RG, Lakatta EG, Le Couteur D, Shaw RJ, Navas P, Puigserver P, Ingram DK, de Cabo R and Sinclair DA. Resveratrol improves health and survival of mice on a high-calorie diet. Nature 444: 337-342, 2006.

17. Beauchamp JR, Heslop L, Yu DS, Tajbakhsh S, Kelly RG, Wernig A, Buckingham ME, Partridge TA and Zammit PS. Expression of CD34 and Myf5 defines the majority of quiescent adult skeletal muscle satellite cells. J Cell Biol 151: 1221-1234, 2000.

18. Beher D, Wu J, Cumine S, Kim KW, Lu SC, Atangan L and Wang M. Resveratrol is not a direct activator of SIRT1 enzyme activity. Chem Biol Drug Des 74: 619-624, 2009.

19. Biolo G, Ciocchi B, Lebenstedt M, Barazzoni R, Zanetti M, Platen P, Heer M and Guarnieri G. Short-term bed rest impairs amino acid-induced protein anabolism in humans. J Physiol 558: 381-388, 2004. 
20. Bober E, Franz T, Arnold HH, Gruss P and Tremblay P. Pax-3 is required for the development of limb muscles: a possible role for the migration of dermomyotomal muscle progenitor cells. Development 120: 603-612, 1994.

21. Bockhold KJ, Rosenblatt JD and Partridge TA. Aging normal and dystrophic mouse muscle: analysis of myogenicity in cultures of living single fibers. Muscle Nerve 21: 173-183, 1998.

22. Boffoli D, Scacco SC, Vergari R, Persio MT, Solarino G, Laforgia R and Papa S. Ageing is associated in females with a decline in the content and activity on the b-c1 complex in skeletal muscle mitochondria. Biochim Biophys Acta 1315: 66-72, 1996.

23. Bokov A, Chaudhuri A and Richardson A. The role of oxidative damage and stress in aging. Mech Ageing Dev 125: 811-826, 2004.

24. Boldrin L and Morgan JE. Activating muscle stem cells: therapeutic potential in muscle diseases. Curr Opin Neurol 20: 577-582, 2007.

25. Boonyarom $\mathbf{O}$ and Inui K. Atrophy and hypertrophy of skeletal muscles: structural and functional aspects. Acta Physiol (Oxf) 188: 77-89, 2006.

26. Bopp D, Burri M, Baumgartner S, Frigerio G and Noll M. Conservation of a large protein domain in the segmentation gene paired and in functionally related genes of Drosophila. Cell 47: 10331040, 1986. 
27. Bortoli S, Renault V, Eveno E, Auffray C, Butler-Browne G and Pietu G. Gene expression profiling of human satellite cells during muscular aging using cDNA arrays. Gene 321: 145-154, 2003.

28. Bottinelli R, Canepari M, Pellegrino MA and Reggiani C. Force-velocity properties of human skeletal muscle fibres: myosin heavy chain isoform and temperature dependence. J Physiol 495 ( Pt 2): 573-586, 1996.

29. Bottinelli R, Canepari M, Reggiani C and Stienen GJ. Myofibrillar ATPase activity during isometric contraction and isomyosin composition in rat single skinned muscle fibres. J Physiol 481 ( Pt 3): 663-675, 1994.

30. Bottinelli R, Schiaffino S and Reggiani C. Force-velocity relations and myosin heavy chain isoform compositions of skinned fibres from rat skeletal muscle. J Physiol 437: 655-672, 1991.

31. Boveris A and Chance B. The mitochondrial generation of hydrogen peroxide. General properties and effect of hyperbaric oxygen. Biochem J 134: 707-716, 1973.

32. Brack AS, Conboy IM, Conboy MJ, Shen J and Rando TA. A temporal switch from notch to Wnt signaling in muscle stem cells is necessary for normal adult myogenesis. Cell Stem Cell 2: 50-59, 2008.

33. Brault JJ, Jespersen JG and Goldberg AL. Peroxisome proliferator-activated receptor gamma coactivator 1alpha or 1beta overexpression inhibits muscle protein degradation, induction of ubiquitin ligases, and disuse atrophy. J Biol Chem 285: 19460-19471, 2010. 
34. Brooks N, Cloutier GJ, Cadena SM, Layne JE, Nelsen CA, Freed AM, Roubenoff R and Castaneda-Sceppa C. Resistance training and timed essential amino acids protect against the loss of muscle mass and strength during 28 days of bed rest and energy deficit. J Appl Physiol 105: 241-248, 2008.

35. Brooks SV. Current topics for teaching skeletal muscle physiology. Adv Physiol Educ 27: 171-182, 2003.

36. Burton LA and Sumukadas D. Optimal management of sarcopenia. Clin Interv Aging 5: 217-228, 2010.

37. Cairns J. Mutation selection and the natural history of cancer. Nature 255: 197-200, 1975.

38. Calleja M, Pena P, Ugalde C, Ferreiro C, Marco R and Garesse R. Mitochondrial DNA remains intact during Drosophila aging, but the levels of mitochondrial transcripts are significantly reduced. J Biol Chem 268: 18891-18897, 1993.

39. Cande C, Vahsen N, Kouranti I, Schmitt E, Daugas E, Spahr C, Luban J, Kroemer RT, Giordanetto F, Garrido C, Penninger JM and Kroemer G. AIF and cyclophilin A cooperate in apoptosisassociated chromatinolysis. Oncogene 23: 1514-1521, 2004.

40. Canepari M, Pellegrino MA, D'Antona G and Bottinelli R. Single muscle fiber properties in aging and disuse. Scand J Med Sci Sports 20: 10-19, 2010. 
41. Canto C, Gerhart-Hines Z, Feige JN, Lagouge M, Noriega L, Milne JC, Elliott PJ, Puigserver P and Auwerx J. AMPK regulates energy expenditure by modulating NAD+ metabolism and SIRT1 activity. Nature 458: 1056-1060, 2009.

42. Canto C, Jiang LQ, Deshmukh AS, Mataki C, Coste A, Lagouge M, Zierath JR and Auwerx J. Interdependence of AMPK and SIRT1 for metabolic adaptation to fasting and exercise in skeletal muscle. Cell Metab 11: 213-219, 2010.

43. Carafa V, Nebbioso A and Altucci L. Sirtuins and disease: the road ahead. Front Pharmacol 3: 4, 2012.

44. Carlson ME, Hsu M and Conboy IM. Imbalance between pSmad3 and Notch induces CDK inhibitors in old muscle stem cells. Nature 454: 528-532, 2008.

45. Carrithers JA, Tesch PA, Trieschmann J, Ekberg A and Trappe TA. Skeletal muscle protein composition following 5 weeks of ULLS and resistance exercise countermeasures. J Gravit Physiol 9: 155-156, 2002.

46. Castillo EM, Goodman-Gruen D, Kritz-Silverstein D, Morton DJ, Wingard DL and BarrettConnor E. Sarcopenia in elderly men and women: the Rancho Bernardo study. Am J Prev Med 25: 226-231, 2003.

47. Chakravarthy MV, Davis BS and Booth FW. IGF-I restores satellite cell proliferative potential in immobilized old skeletal muscle. J Appl Physiol 89: 1365-1379, 2000. 
48. Chance B, Leigh JS, Jr., Clark BJ, Maris J, Kent J, Nioka S and Smith D. Control of oxidative metabolism and oxygen delivery in human skeletal muscle: a steady-state analysis of the work/energy cost transfer function. Proc Natl Acad Sci U S A 82: 8384-8388, 1985.

49. Chao DT and Korsmeyer SJ. BCL-2 family: regulators of cell death. Annu Rev Immunol 16: 395419, 1998.

50. Charge SB, Brack AS and Hughes SM. Aging-related satellite cell differentiation defect occurs prematurely after Ski-induced muscle hypertrophy. Am J Physiol Cell Physiol 283: C1228-C1241, 2002.

51. Charge SB and Rudnicki MA. Cellular and molecular regulation of muscle regeneration. Physiol $\operatorname{Rev} 84:$ 209-238, 2004.

52. Charge SB and Rudnicki MA. Cellular and molecular regulation of muscle regeneration. Physiol Rev 84: 209-238, 2004.

53. Chen KD and Alway SE. Clenbuterol reduces soleus muscle fatigue during disuse in aged rats. Muscle Nerve 24: 211-222, 2001.

54. Chi TC, Chen WP, Chi TL, Kuo TF, Lee SS, Cheng JT and Su MJ. Phosphatidylinositol-3-kinase is involved in the antihyperglycemic effect induced by resveratrol in streptozotocin-induced diabetic rats. Life Sci 80: 1713-1720, 2007. 
55. Chipuk JE, Bouchier-Hayes L and Green DR. Mitochondrial outer membrane permeabilization during apoptosis: the innocent bystander scenario. Cell Death Differ 13: 1396-1402, 2006.

56. Chipuk JE and Green DR. How do BCL-2 proteins induce mitochondrial outer membrane permeabilization? Trends Cell Biol 18: 157-164, 2008.

57. Ciciliot S and Schiaffino S. Regeneration of mammalian skeletal muscle. Basic mechanisms and clinical implications. Curr Pharm Des 16: 906-914, 2010.

58. Cohen HY, Miller C, Bitterman KJ, Wall NR, Hekking B, Kessler B, Howitz KT, Gorospe M, de Cabo $\mathbf{R}$ and Sinclair DA. Calorie restriction promotes mammalian cell survival by inducing the SIRT1 deacetylase. Science 305: 390-392, 2004.

59. Collins CA, Olsen I, Zammit PS, Heslop L, Petrie A, Partridge TA and Morgan JE. Stem cell function, self-renewal, and behavioral heterogeneity of cells from the adult muscle satellite cell niche. Cell 122: 289-301, 2005.

60. Collins CA, Zammit PS, Ruiz AP, Morgan JE and Partridge TA. A population of myogenic stem cells that survives skeletal muscle aging. Stem Cells 25: 885-894, 2007.

61. Conboy IM, Conboy MJ, Smythe GM and Rando TA. Notch-mediated restoration of regenerative potential to aged muscle. Science 302: 1575-1577, 2003.

62. Conboy IM and Rando TA. The regulation of Notch signaling controls satellite cell activation and cell fate determination in postnatal myogenesis. Dev Cell 3: 397-409, 2002. 
63. Conley KE, Jubrias SA and Esselman PC. Oxidative capacity and ageing in human muscle. J Physiol 526 Pt 1: 203-210, 2000.

64. Cornelison DD, Filla MS, Stanley HM, Rapraeger AC and Olwin BB. Syndecan-3 and syndecan-4 specifically mark skeletal muscle satellite cells and are implicated in satellite cell maintenance and muscle regeneration. Dev Biol 239: 79-94, 2001.

65. Cornelison DD, Olwin BB, Rudnicki MA and Wold BJ. MyoD(-/-) satellite cells in single-fiber culture are differentiation defective and MRF4 deficient. Dev Biol 224: 122-137, 2000.

66. Cornelison DD and Wold BJ. Single-cell analysis of regulatory gene expression in quiescent and activated mouse skeletal muscle satellite cells. Dev Biol 191: 270-283, 1997.

67. Cruz-Jentoft AJ, Baeyens JP, Bauer JM, Boirie Y, Cederholm T, Landi F, Martin FC, Michel JP, Rolland Y, Schneider SM, Topinkova E, Vandewoude M and Zamboni M. Sarcopenia: European consensus on definition and diagnosis: Report of the European Working Group on Sarcopenia in Older People. Age Ageing 39: 412-423, 2010.

68. Cruz-Jentoft AJ, Baeyens JP, Bauer JM, Boirie Y, Cederholm T, Landi F, Martin FC, Michel JP, Rolland Y, Schneider SM, Topinkova E, Vandewoude M and Zamboni M. Sarcopenia: European consensus on definition and diagnosis: Report of the European Working Group on Sarcopenia in Older People. Age Ageing 39: 412-423, 2010. 
69. d'Albis A, Couteaux R, Janmot C, Roulet A and Mira JC. Regeneration after cardiotoxin injury of innervated and denervated slow and fast muscles of mammals. Myosin isoform analysis. Eur J Biochem 174: 103-110, 1988.

70. D'Antona G, Pellegrino MA, Adami R, Rossi R, Carlizzi CN, Canepari M, Saltin B and Bottinelli R. The effect of ageing and immobilization on structure and function of human skeletal muscle fibres. J Physio/ 552: 499-511, 2003.

71. Danial NN and Korsmeyer SJ. Cell death: critical control points. Cell 116: 205-219, 2004.

72. Daugas E, Nochy D, Ravagnan L, Loeffler M, Susin SA, Zamzami N and Kroemer G. Apoptosisinducing factor (AIF): a ubiquitous mitochondrial oxidoreductase involved in apoptosis. FEBS Lett 476: 118-123, 2000.

73. de Boer MD, Selby A, Atherton P, Smith K, Seynnes OR, Maganaris CN, Maffulli N, Movin T, Narici MV and Rennie MJ. The temporal responses of protein synthesis, gene expression and cell signalling in human quadriceps muscle and patellar tendon to disuse. J Physiol 585: 241-251, 2007.

74. de Boer MD, Seynnes OR, di Prampero PE, Pisot R, Mekjavic IB, Biolo G and Narici MV. Effect of 5 weeks horizontal bed rest on human muscle thickness and architecture of weight bearing and non-weight bearing muscles. Eur J Appl Physiol 104: 401-407, 2008.

75. de la Lastra CA and Villegas I. Resveratrol as an anti-inflammatory and anti-aging agent: mechanisms and clinical implications. Mol Nutr Food Res 49: 405-430, 2005. 
76. de Moura MB, dos Santos LS and Van Houten B. Mitochondrial dysfunction in neurodegenerative diseases and cancer. Environ Mol Mutagen 51: 391-405, 2010.

77. Deng JY, Hsieh PS, Huang JP, Lu LS and Hung LM. Activation of estrogen receptor is crucial for resveratrol-stimulating muscular glucose uptake via both insulin-dependent and -independent pathways. Diabetes 57: 1814-1823, 2008.

78. Deschenes MR, Giles JA, McCoy RW, Volek JS, Gomez AL and Kraemer WJ. Neural factors account for strength decrements observed after short-term muscle unloading. Am J Physiol Regul Integr Comp Physiol 282: R578-R583, 2002.

79. Dodd SL and Koesterer TJ. Clenbuterol attenuates muscle atrophy and dysfunction in hindlimbsuspended rats. Aviat Space Environ Med 73: 635-639, 2002.

80. Dolinsky VW, Jones KE, Sidhu RS, Haykowsky M, Czubryt MP, Gordon T and Dyck JR. Improvements in skeletal muscle strength and cardiac function induced by resveratrol during exercise training contribute to enhanced exercise performance in rats. J Physiol 590: 2783-2799, 2012.

81. Drey M. Sarcopenia - pathophysiology and clinical relevance. Wien Med Wochenschr 2011.

82. Droge W. Free radicals in the physiological control of cell function. Physiol Rev 82: 47-95, 2002.

83. Dupont Salter AC, Richmond FJ and Loeb GE. Prevention of muscle disuse atrophy by lowfrequency electrical stimulation in rats. IEEE Trans Neural Syst Rehabil Eng 11: 218-226, 2003. 
84. Dupont-Versteegden EE. Apoptosis in muscle atrophy: relevance to sarcopenia. Exp Gerontol 40: 473-481, 2005.

85. Erim Z, Beg MF, Burke DT and de Luca CJ. Effects of aging on motor-unit control properties. J Neurophysiol 82: 2081-2091, 1999.

86. Ferrieres J. The French paradox: lessons for other countries. Heart 90: 107-111, 2004.

87. Frontera WR, Hughes VA, Fielding RA, Fiatarone MA, Evans WJ and Roubenoff R. Aging of skeletal muscle: a 12-yr longitudinal study. J Appl Physiol 88: 1321-1326, 2000.

88. Fulco M, Cen Y, Zhao P, Hoffman EP, McBurney MW, Sauve AA and Sartorelli V. Glucose restriction inhibits skeletal myoblast differentiation by activating SIRT1 through AMPK-mediated regulation of Nampt. Dev Cell 14: 661-673, 2008.

89. Fulco M, Schiltz RL, lezzi S, King MT, Zhao P, Kashiwaya $Y$, Hoffman E, Veech RL and Sartorelli V. Sir2 regulates skeletal muscle differentiation as a potential sensor of the redox state. Mol Cell 12: 51-62, 2003.

90. Gayraud-Morel B, Chretien F, Flamant P, Gomes D, Zammit PS and Tajbakhsh S. A role for the myogenic determination gene Myf5 in adult regenerative myogenesis. Dev Biol 312: 13-28, 2007.

91. Gelfi C, Vasso M and Cerretelli P. Diversity of human skeletal muscle in health and disease: contribution of proteomics. J Proteomics 74: 774-795, 2011. 
92. Gerhart-Hines Z, Rodgers JT, Bare O, Lerin C, Kim SH, Mostoslavsky R, Alt FW, Wu Z and Puigserver P. Metabolic control of muscle mitochondrial function and fatty acid oxidation through SIRT1/PGC-1alpha. EMBO J 26: 1913-1923, 2007.

93. Goulding $\mathbf{M}$, Lumsden $\mathbf{A}$ and Paquette $\mathbf{A J}$. Regulation of Pax-3 expression in the dermomyotome and its role in muscle development. Development 120: 957-971, 1994.

94. Gu J, Song ZP, Gui DM, Hu W, Chen YG and Zhang DD. Resveratrol Attenuates DoxorubicinInduced Cardiomyocyte Apoptosis in Lymphoma Nude Mice by Heme Oxygenase-1 Induction. Cardiovasc Toxicol 2012.

95. Habold C, Momken I, Ouadi A, Bekaert V and Brasse D. Effect of prior treatment with resveratrol on density and structure of rat long bones under tail-suspension. J Bone Miner Metab 29: 15-22, 2011.

96. Hall-Craggs EC. Rapid degeneration and regeneration of a whole skeletal muscle following treatment with bupivacaine (Marcain). Exp Neurol 43: 349-358, 1974.

97. Han B, Zhu MJ, Ma C and Du M. Rat hindlimb unloading down-regulates insulin like growth factor-1 signaling and AMP-activated protein kinase, and leads to severe atrophy of the soleus muscle. Appl Physiol Nutr Metab 32: 1115-1123, 2007.

98. Hancock K, Chang E, Chenoweth L, Clarke M, Carroll A and Jeon YH. Nursing needs of acutely ill older people. J Adv Nurs 44: 507-516, 2003. 


\section{Handschin C, Chin S, Li P, Liu F, Maratos-Flier E, Lebrasseur NK, Yan Z and Spiegelman BM.}

Skeletal muscle fiber-type switching, exercise intolerance, and myopathy in PGC-1alpha musclespecific knock-out animals. J Biol Chem 282: 30014-30021, 2007.

100. Hao Y, Jackson JR, Wang Y, Edens N, Pereira SL and Alway SE. beta-Hydroxy-betamethylbutyrate reduces myonuclear apoptosis during recovery from hind limb suspensioninduced muscle fiber atrophy in aged rats. Am J Physiol Regul Integr Comp Physiol 301: R701R715, 2011.

101. Hao Y, Jackson JR, Wang Y, Edens N, Pereira SL and Alway SE. beta-Hydroxy-betamethylbutyrate reduces myonuclear apoptosis during recovery from hind limb suspensioninduced muscle fiber atrophy in aged rats. Am J Physiol Regul Integr Comp Physiol 301: R701R715, 2011.

102. Harikumar KB and Aggarwal BB. Resveratrol: a multitargeted agent for age-associated chronic diseases. Cell Cycle 7: 1020-1035, 2008.

103. HARMAN D. Aging: a theory based on free radical and radiation chemistry. J Gerontol 11: 298300, 1956.

104. Harris JB and Johnson MA. Further observations on the pathological responses of rat skeletal muscle to toxins isolated from the venom of the Australian tiger snake, Notechis scutatus scutatus. Clin Exp Pharmacol Physiol 5: 587-600, 1978. 
105. Hawley SA, Ross FA, Chevtzoff C, Green KA, Evans A, Fogarty S, Towler MC, Brown L, Ogunbayo OA, Evans AM and Hardie DG. Use of cells expressing gamma subunit variants to identify diverse mechanisms of AMPK activation. Cell Metab 11: 554-565, 2010.

106. Hengartner MO. The biochemistry of apoptosis. Nature 407: 770-776, 2000.

107. Herranz D and Serrano M. SIRT1: recent lessons from mouse models. Nat Rev Cancer 10: 819823, 2010.

108. Herranz D and Serrano M. SIRT1: recent lessons from mouse models. Nat Rev Cancer 10: 819823, 2010.

109. Hespel P, Op't EB, Van Leemputte M, Urso B, Greenhaff PL, Labarque V, Dymarkowski S, Van Hecke P and Richter EA. Oral creatine supplementation facilitates the rehabilitation of disuse atrophy and alters the expression of muscle myogenic factors in humans. J Physio/ 536: 625-633, 2001.

110. Hortobagyi T, Dempsey L, Fraser D, Zheng D, Hamilton G, Lambert J and Dohm L. Changes in muscle strength, muscle fibre size and myofibrillar gene expression after immobilization and retraining in humans. J Physiol 524 Pt 1: 293-304, 2000.

111. Houmard JA, Weidner ML, Gavigan KE, Tyndall GL, Hickey MS and Alshami A. Fiber type and citrate synthase activity in the human gastrocnemius and vastus lateralis with aging. J Appl Physiol 85: 1337-1341, 1998. 
112. Hu Z, Wang H, Lee IH, Modi S, Wang X, Du J and Mitch WE. PTEN inhibition improves muscle regeneration in mice fed a high-fat diet. Diabetes 59: 1312-1320, 2010.

113. Huang KJ, Ku CC and Lehman IR. Endonuclease G: a role for the enzyme in recombination and cellular proliferation. Proc Natl Acad Sci U S A 103: 8995-9000, 2006.

114. Huard J, Li Y and Fu FH. Muscle injuries and repair: current trends in research. J Bone Joint Surg Am 84-A: 822-832, 2002.

115. Huffman DM, Moellering DR, Grizzle WE, Stockard CR, Johnson MS and Nagy TR. Effect of exercise and calorie restriction on biomarkers of aging in mice. Am J Physiol Regul Integr Comp Physiol 294: R1618-R1627, 2008.

116. Hur YG, Suh CH, Kim S and Won J. Rosmarinic acid induces apoptosis of activated T cells from rheumatoid arthritis patients via mitochondrial pathway. J Clin Immunol 27: 36-45, 2007.

117. Hwee DT and Bodine SC. Age-related deficit in load-induced skeletal muscle growth. J Gerontol A Biol Sci Med Sci 64: 618-628, 2009.

118. Inouye SK, Bogardus ST, Jr., Baker DI, Leo-Summers L and Cooney LM, Jr. The Hospital Elder Life Program: a model of care to prevent cognitive and functional decline in older hospitalized patients. Hospital Elder Life Program. J Am Geriatr Soc 48: 1697-1706, 2000.

119. Irintchev A and Wernig A. Muscle damage and repair in voluntarily running mice: strain and muscle differences. Cell Tissue Res 249: 509-521, 1987. 
120. Irintchev A, Zeschnigk M, Starzinski-Powitz A and Wernig A. Expression pattern of M-cadherin in normal, denervated, and regenerating mouse muscles. Dev Dyn 199: 326-337, 1994.

121. Itai Y, Kariya $\mathbf{Y}$ and Hoshino Y. Morphological changes in rat hindlimb muscle fibres during recovery from disuse atrophy. Acta Physiol Scand 181: 217-224, 2004.

122. Jackson JR, Ryan MJ and Alway SE. Long-term supplementation with resveratrol alleviates oxidative stress but does not attenuate sarcopenia in aged mice. J Gerontol A Biol Sci Med Sci 66: 751-764, 2011.

123. Jackson JR, Ryan MJ, Hao Y and Alway SE. Mediation of endogenous antioxidant enzymes and apoptotic signaling by resveratrol following muscle disuse in the gastrocnemius muscles of young and old rats. Am J Physiol Regul Integr Comp Physiol 299: R1572-R1581, 2010.

124. Jackson MJ. Redox regulation of adaptive responses in skeletal muscle to contractile activity. Free Radic Biol Med 47: 1267-1275, 2009.

125. Jackson MJ, Papa S, Bolanos J, Bruckdorfer R, Carlsen H, Elliott RM, Flier J, Griffiths HR, Heales S, Holst B, Lorusso M, Lund E, Oivind MJ, Moser U, Di Paola M, Polidori MC, Signorile A, Stahl W, Vina-Ribes J and Astley SB. Antioxidants, reactive oxygen and nitrogen species, gene induction and mitochondrial function. Mol Aspects Med 23: 209-285, 2002.

126. Janssen I, Heymsfield SB, Wang ZM and Ross R. Skeletal muscle mass and distribution in 468 men and women aged 18-88 yr. J Appl Physiol 89: 81-88, 2000. 
127. Janssen I, Shepard DS, Katzmarzyk PT and Roubenoff R. The healthcare costs of sarcopenia in the United States. J Am Geriatr Soc 52: 80-85, 2004.

128. Jeong SY and Seol DW. The role of mitochondria in apoptosis. BMB Rep 41: 11-22, 2008.

129. Jones SW, Hill RJ, Krasney PA, O'Conner B, Peirce N and Greenhaff PL. Disuse atrophy and exercise rehabilitation in humans profoundly affects the expression of genes associated with the regulation of skeletal muscle mass. FASEB J 18: 1025-1027, 2004.

130. Kaeberlein M, McVey $\mathbf{M}$ and Guarente L. The SIR2/3/4 complex and SIR2 alone promote longevity in Saccharomyces cerevisiae by two different mechanisms. Genes Dev 13: 2570-2580, 1999.

131. Karagounis LG and Hawley JA. Skeletal muscle: increasing the size of the locomotor cell. Int J Biochem Cell Biol 42: 1376-1379, 2010.

132. Kent-Braun JA and Ng AV. Specific strength and voluntary muscle activation in young and elderly women and men. J Appl Physiol 87: 22-29, 1999.

133. Kerr JF, Wyllie AH and Currie AR. Apoptosis: a basic biological phenomenon with wide-ranging implications in tissue kinetics. Br J Cancer 26: 239-257, 1972.

134. Knapp JR, Davie JK, Myer A, Meadows E, Olson EN and Klein WH. Loss of myogenin in postnatal life leads to normal skeletal muscle but reduced body size. Development 133: 601-610, 2006. 
135. Koishi K, Zhang M, McLennan IS and Harris AJ. MyoD protein accumulates in satellite cells and is neurally regulated in regenerating myotubes and skeletal muscle fibers. Dev Dyn 202: 244254, 1995.

136. Koltai E, Szabo Z, Atalay M, Boldogh I, Naito H, Goto S, Nyakas C and Radak Z. Exercise alters SIRT1, SIRT6, NAD and NAMPT levels in skeletal muscle of aged rats. Mech Ageing Dev 131: 21$28,2010$.

137. Kuang S, Charge SB, Seale P, Huh M and Rudnicki MA. Distinct roles for Pax7 and Pax3 in adult regenerative myogenesis. J Cell Biol 172: 103-113, 2006.

138. Kuang S, Kuroda K, Le Grand F and Rudnicki MA. Asymmetric self-renewal and commitment of satellite stem cells in muscle. Cell 129: 999-1010, 2007.

139. Lagouge M, Argmann C, Gerhart-Hines Z, Meziane H, Lerin C, Daussin F, Messadeq N, Milne J, Lambert P, Elliott P, Geny B, Laakso M, Puigserver P and Auwerx J. Resveratrol improves mitochondrial function and protects against metabolic disease by activating SIRT1 and PGC1alpha. Cell 127: 1109-1122, 2006.

140. Lanza IR and Sreekumaran NK. Regulation of skeletal muscle mitochondrial function: genes to proteins. Acta Physiol (Oxf) 199: 529-547, 2010.

141. Larsson L, Li $\mathbf{X}$ and Frontera WR. Effects of aging on shortening velocity and myosin isoform composition in single human skeletal muscle cells. Am J Physiol 272: C638-C649, 1997. 
142. Le Grand F, Jones AE, Seale V, Scime A and Rudnicki MA. Wnt7a activates the planar cell polarity pathway to drive the symmetric expansion of satellite stem cells. Cell Stem Cell 4: 535$547,2009$.

143. Le Grand F and Rudnicki MA. Skeletal muscle satellite cells and adult myogenesis. Curr Opin Cell Biol 19: 628-633, 2007.

144. Leeuwenburgh C, Gurley CM, Strotman BA and Dupont-Versteegden EE. Age-related differences in apoptosis with disuse atrophy in soleus muscle. Am J Physiol Regul Integr Comp Physiol 288: R1288-R1296, 2005.

145. Lefaucheur JP and Sebille A. The cellular events of injured muscle regeneration depend on the nature of the injury. Neuromuscul Disord 5: 501-509, 1995.

146. Leiro J, Arranz JA, Parama A, Alvarez MF and Sanmartin ML. In vitro effects of the polyphenols resveratrol, mangiferin and (-)-epigallocatechin-3-gallate on the scuticociliate fish pathogen Philasterides dicentrarchi. Dis Aquat Organ 59: 171-174, 2004.

147. Lepper C, Partridge TA and Fan CM. An absolute requirement for Pax7-positive satellite cells in acute injury-induced skeletal muscle regeneration. Development 138: 3639-3646, 2011.

148. Li H, Rajendran GK, Liu N, Ware C, Rubin BP and Gu Y. SirT1 modulates the estrogen-insulin-like growth factor-1 signaling for postnatal development of mammary gland in mice. Breast Cancer Res 9: R1, 2007. 
149. Li H, Zhu H, Xu CJ and Yuan J. Cleavage of BID by caspase 8 mediates the mitochondrial damage in the Fas pathway of apoptosis. Cell 94: 491-501, 1998.

150. Li LY, Luo $\mathbf{X}$ and Wang $X$. Endonuclease $G$ is an apoptotic DNase when released from mitochondria. Nature 412: 95-99, 2001.

151. Lin J, Wu H, Tarr PT, Zhang CY, Wu Z, Boss O, Michael LF, Puigserver P, Isotani E, Olson EN, Lowell BB, Bassel-Duby R and Spiegelman BM. Transcriptional co-activator PGC-1 alpha drives the formation of slow-twitch muscle fibres. Nature 418: 797-801, 2002.

152. Lindle RS, Metter EJ, Lynch NA, Fleg JL, Fozard JL, Tobin J, Roy TA and Hurley BF. Age and gender comparisons of muscle strength in 654 women and men aged 20-93 yr. J Appl Physiol 83: 1581-1587, 1997.

153. Longo VD. Linking sirtuins, IGF-I signaling, and starvation. Exp Gerontol 44: 70-74, 2009.

154. Longo VD and Kennedy BK. Sirtuins in aging and age-related disease. Cell 126: 257-268, 2006.

155. Loschen G, Azzi A, Richter C and Flohe L. Superoxide radicals as precursors of mitochondrial hydrogen peroxide. FEBS Lett 42: 68-72, 1974.

156. Lu A, Cummins JH, Pollett JB, Cao B, Sun B, Rudnicki MA and Huard J. Isolation of myogenic progenitor populations from Pax7-deficient skeletal muscle based on adhesion characteristics. Gene Ther 15: 1116-1125, 2008. 
157. Machida S and Booth FW. Increased nuclear proteins in muscle satellite cells in aged animals as compared to young growing animals. Exp Gerontol 39: 1521-1525, 2004.

158. Magne H, Savary-Auzeloux I, Vazeille E, Claustre A, Attaix D, Anne L, Veronique SL, Philippe G, Dardevet $\mathbf{D}$ and Combaret $\mathbf{L}$. Lack of muscle recovery after immobilization in old rats does not result from a defect in normalization of the ubiquitin-proteasome and the caspase-dependent apoptotic pathways. J Physiol 589: 511-524, 2011.

159. Mansouri A, Goudreau G and Gruss P. Pax genes and their role in organogenesis. Cancer Res 59: 1707s-1709s, 1999.

160. Mansouri A, Stoykova A, Torres M and Gruss P. Dysgenesis of cephalic neural crest derivatives in Pax7-/- mutant mice. Development 122: 831-838, 1996.

161. Mantel C and Broxmeyer HE. Sirtuin 1, stem cells, aging, and stem cell aging. Curr Opin Hematol 15: 326-331, 2008.

162. Marzetti E, Hwang JC, Lees HA, Wohlgemuth SE, Dupont-Versteegden EE, Carter CS, Bernabei R and Leeuwenburgh C. Mitochondrial death effectors: relevance to sarcopenia and disuse muscle atrophy. Biochim Biophys Acta 1800: 235-244, 2010.

163. MAURO A. Satellite cell of skeletal muscle fibers. J Biophys Biochem Cytol 9: 493-495, 1961.

164. Megeney LA, Kablar B, Garrett K, Anderson JE and Rudnicki MA. MyoD is required for myogenic stem cell function in adult skeletal muscle. Genes Dev 10: 1173-1183, 1996. 
165. Meng SJ and Yu L. Oxidative stress, molecular inflammation and sarcopenia. Int J Mol Sci 11: 1509-1526, 2010.

166. Menshikova EV, Ritov VB, Fairfull L, Ferrell RE, Kelley DE and Goodpaster BH. Effects of exercise on mitochondrial content and function in aging human skeletal muscle. J Gerontol A Biol Sci Med Sci 61: 534-540, 2006.

167. Metter EJ, Conwit R, Tobin J and Fozard JL. Age-associated loss of power and strength in the upper extremities in women and men. J Gerontol A Biol Sci Med Sci 52: B267-B276, 1997.

168. Metter EJ, Talbot LA, Schrager M and Conwit R. Skeletal muscle strength as a predictor of allcause mortality in healthy men. J Gerontol A Biol Sci Med Sci 57: B359-B365, 2002.

169. Mezey M, Kobayashi M, Grossman S, Firpo A, Fulmer T and Mitty E. Nurses Improving Care to Health System Elders (NICHE): implementation of best practice models. J Nurs Adm 34: 451-457, 2004.

170. Mitchell CA, McGeachie JK and Grounds MD. Cellular differences in the regeneration of murine skeletal muscle: a quantitative histological study in SJL/J and BALB/c mice. Cell Tissue Res 269: 159-166, 1992.

171. Momken I, Stevens L, Bergouignan A, Desplanches D, Rudwill F, Chery I, Zahariev A, Zahn S, Stein TP, Sebedio JL, Pujos-Guillot E, Falempin M, Simon C, Coxam V, Andrianjafiniony T, Gauquelin-Koch G, Picquet F and Blanc S. Resveratrol prevents the wasting disorders of 
mechanical unloading by acting as a physical exercise mimetic in the rat. FASEB J 25: 3646-3660, 2011.

172. Momken I, Stevens L, Bergouignan A, Desplanches D, Rudwill F, Chery I, Zahariev A, Zahn S, Stein TP, Sebedio JL, Pujos-Guillot E, Falempin M, Simon C, Coxam V, Andrianjafiniony T, Gauquelin-Koch G, Picquet F and Blanc S. Resveratrol prevents the wasting disorders of mechanical unloading by acting as a physical exercise mimetic in the rat. FASEB J 25: 3646-3660, 2011.

173. Moss FP and Leblond CP. Nature of dividing nuclei in skeletal muscle of growing rats. J Cell Biol 44: 459-462, 1970.

174. Moss FP and Leblond CP. Satellite cells as the source of nuclei in muscles of growing rats. Anat $\operatorname{Rec}$ 170: 421-435, 1971.

175. Musaro A, McCullagh K, Paul A, Houghton L, Dobrowolny G, Molinaro M, Barton ER, Sweeney HL and Rosenthal N. Localized Igf-1 transgene expression sustains hypertrophy and regeneration in senescent skeletal muscle. Nat Genet 27: 195-200, 2001.

176. Nagata Y, Partridge TA, Matsuda R and Zammit PS. Entry of muscle satellite cells into the cell cycle requires sphingolipid signaling. J Cell Biol 174: 245-253, 2006.

177. Narici MV, Bordini M and Cerretelli P. Effect of aging on human adductor pollicis muscle function. J Appl Physiol 71: 1277-1281, 1991. 
178. Narici MV, Reeves ND, Morse $\mathrm{Cl}$ and Maganaris CN. Muscular adaptations to resistance exercise in the elderly. J Musculoskelet Neuronal Interact 4: 161-164, 2004.

179. Nemoto S, Fergusson MM and Finkel T. SIRT1 functionally interacts with the metabolic regulator and transcriptional coactivator PGC-1\{alpha\}. J Biol Chem 280: 16456-16460, 2005.

180. Newman AB, Kupelian V, Visser M, Simonsick EM, Goodpaster BH, Kritchevsky SB, Tylavsky FA, Rubin SM and Harris TB. Strength, but not muscle mass, is associated with mortality in the health, aging and body composition study cohort. J Gerontol A Biol Sci Med Sci 61: 72-77, 2006.

181. Ohira Y, Jiang B, Roy RR, Oganov V, llyina-Kakueva E, Marini JF and Edgerton VR. Rat soleus muscle fiber responses to 14 days of spaceflight and hindlimb suspension. J App/ Physiol 73: 51S57S, 1992.

182. Otto A, Collins-Hooper $\mathbf{H}$ and Patel K. The origin, molecular regulation and therapeutic potential of myogenic stem cell populations. J Anat 215: 477-497, 2009.

183. Oustanina S, Hause G and Braun T. Pax7 directs postnatal renewal and propagation of myogenic satellite cells but not their specification. EMBO J 23: 3430-3439, 2004.

184. Pacholec M, Bleasdale JE, Chrunyk B, Cunningham D, Flynn D, Garofalo RS, Griffith D, Griffor M, Loulakis P, Pabst B, Qiu X, Stockman B, Thanabal V, Varghese A, Ward J, Withka J and Ahn K. SRT1720, SRT2183, SRT1460, and resveratrol are not direct activators of SIRT1. J Biol Chem 285: 8340-8351, 2010. 
185. Pardo PS and Boriek AM. The physiological roles of Sirt1 in skeletal muscle. Aging (Albany NY) 3: 430-437, 2011.

186. Park CE, Kim MJ, Lee JH, Min BI, Bae H, Choe W, Kim SS and Ha J. Resveratrol stimulates glucose transport in C2C12 myotubes by activating AMP-activated protein kinase. Exp Mol Med 39: 222-229, 2007.

187. Pastoris O, Boschi F, Verri M, Baiardi P, Felzani G, Vecchiet J, Dossena M and Catapano M. The effects of aging on enzyme activities and metabolite concentrations in skeletal muscle from sedentary male and female subjects. Exp Gerontol 35: 95-104, 2000.

188. Pavlath GK, Thaloor D, Rando TA, Cheong M, English AW and Zheng B. Heterogeneity among muscle precursor cells in adult skeletal muscles with differing regenerative capacities. Dev Dyn 212: 495-508, 1998.

189. Pelosi L, Giacinti C, Nardis C, Borsellino G, Rizzuto E, Nicoletti C, Wannenes F, Battistini L, Rosenthal N, Molinaro M and Musaro A. Local expression of IGF-1 accelerates muscle regeneration by rapidly modulating inflammatory cytokines and chemokines. FASEB J 21: 1393$1402,2007$.

190. Pesce V, Cormio A, Fracasso F, Lezza AM, Cantatore P and Gadaleta MN. Rat hindlimb unloading: Soleus and Extensor Digitorum Longus histochemistry, mitochondrial DNA content and mitochondrial DNA deletions. Biosci Rep 22: 115-125, 2002. 
191. Petersen KF, Befroy D, Dufour S, Dziura J, Ariyan C, Rothman DL, DiPietro L, Cline GW and Shulman GI. Mitochondrial dysfunction in the elderly: possible role in insulin resistance. Science 300: 1140-1142, 2003.

192. Pierno S, Desaphy JF, Liantonio A, De Bellis M, Bianco G, De Luca A, Frigeri A, Nicchia GP, Svelto M, Leoty C, George AL, Jr. and Camerino DC. Change of chloride ion channel conductance is an early event of slow-to-fast fibre type transition during unloading-induced muscle disuse. Brain 125: 1510-1521, 2002.

193. Pistilli EE, Siu PM and Alway SE. Molecular regulation of apoptosis in fast plantaris muscles of aged rats. J Gerontol A Biol Sci Med Sci 61: 245-255, 2006.

194. Pistilli EE, Siu PM and Alway SE. Interleukin-15 responses to aging and unloading-induced skeletal muscle atrophy. Am J Physiol Cell Physiol 292: C1298-C1304, 2007.

195. Powers SK, Kavazis AN and DeRuisseau KC. Mechanisms of disuse muscle atrophy: role of oxidative stress. Am J Physiol Regul Integr Comp Physiol 288: R337-R344, 2005.

196. Rahman I, Biswas SK and Kirkham PA. Regulation of inflammation and redox signaling by dietary polyphenols. Biochem Pharmacol 72: 1439-1452, 2006.

197. Rasmussen UF, Krustrup P, Kjaer M and Rasmussen HN. Human skeletal muscle mitochondrial metabolism in youth and senescence: no signs of functional changes in ATP formation and mitochondrial oxidative capacity. Pflugers Arch 446: 270-278, 2003. 
198. Rathbone CR, Booth FW and Lees SJ. Sirt1 increases skeletal muscle precursor cell proliferation. Eur J Cell Biol 88: 35-44, 2009.

199. Relaix F, Montarras D, Zaffran S, Gayraud-Morel B, Rocancourt D, Tajbakhsh S, Mansouri A, Cumano A and Buckingham M. Pax3 and Pax7 have distinct and overlapping functions in adult muscle progenitor cells. J Cell Biol 172: 91-102, 2006.

200. Reznik M. Thymidine-3H uptake by satellite cells of regenerating skeletal muscle. J Cell Biol 40: 568-571, 1969.

201. Riley DA, Slocum GR, Bain JL, Sedlak FR, Sowa TE and Mellender JW. Rat hindlimb unloading: soleus histochemistry, ultrastructure, and electromyography. J Appl Physiol 69: 58-66, 1990.

202. Ristow M, Zarse K, Oberbach A, Kloting N, Birringer M, Kiehntopf M, Stumvoll M, Kahn CR and Bluher M. Antioxidants prevent health-promoting effects of physical exercise in humans. Proc Natl Acad Sci U S A 106: 8665-8670, 2009.

203. Rooyackers OE, Adey DB, Ades PA and Nair KS. Effect of age on in vivo rates of mitochondrial protein synthesis in human skeletal muscle. Proc Natl Acad Sci U S A 93: 15364-15369, 1996.

204. Roy RR, Baldwin KM and Edgerton VR. Response of the neuromuscular unit to spaceflight: what has been learned from the rat model. Exerc Sport Sci Rev 24: 399-425, 1996.

205. Russell ST, Wyke SM and Tisdale MJ. Mechanism of induction of muscle protein degradation by angiotensin II. Cell Signal 18: 1087-1096, 2006. 
206. Ryan MJ, Jackson JR, Hao Y, Williamson CL, Dabkowski ER, Hollander JM and Alway SE.

Suppression of oxidative stress by resveratrol after isometric contractions in gastrocnemius muscles of aged mice. J Gerontol A Biol Sci Med Sci 65: 815-831, 2010.

207. Sambasivan R, Yao R, Kissenpfennig A, Van Wittenberghe L, Paldi A, Gayraud-Morel B, Guenou H, Malissen B, Tajbakhsh S and Galy A. Pax7-expressing satellite cells are indispensable for adult skeletal muscle regeneration. Development 138: 3647-3656, 2011.

208. Sandri M, Lin J, Handschin C, Yang W, Arany ZP, Lecker SH, Goldberg AL and Spiegelman BM. PGC-1alpha protects skeletal muscle from atrophy by suppressing FoxO3 action and atrophyspecific gene transcription. Proc Natl Acad Sci U S A 103: 16260-16265, 2006.

209. Schultz E and Lipton BH. Skeletal muscle satellite cells: changes in proliferation potential as a function of age. Mech Ageing Dev 20: 377-383, 1982.

210. Schulze PC, Fang J, Kassik KA, Gannon J, Cupesi M, MacGillivray C, Lee RT and Rosenthal N. Transgenic overexpression of locally acting insulin-like growth factor-1 inhibits ubiquitinmediated muscle atrophy in chronic left-ventricular dysfunction. Circ Res 97: 418-426, 2005.

211. Seale P, Sabourin LA, Girgis-Gabardo A, Mansouri A, Gruss P and Rudnicki MA. Pax7 is required for the specification of myogenic satellite cells. Cell 102: 777-786, 2000.

212. Selsby JT, Morine KJ, Pendrak K, Barton ER and Sweeney HL. Rescue of dystrophic skeletal muscle by PGC-1alpha involves a fast to slow fiber type shift in the mdx mouse. PLoS One 7: e30063, 2012. 
213. Shakibaei M, Harikumar KB and Aggarwal BB. Resveratrol addiction: to die or not to die. Mol Nutr Food Res 53: 115-128, 2009.

214. Shefer G, Van de Mark DP, Richardson JB and Yablonka-Reuveni Z. Satellite-cell pool size does matter: defining the myogenic potency of aging skeletal muscle. Dev Biol 294: 50-66, 2006.

215. Shinin V, Gayraud-Morel B, Gomes D and Tajbakhsh S. Asymmetric division and cosegregation of template DNA strands in adult muscle satellite cells. Nat Cell Biol 8: 677-687, 2006.

216. Short KR, Bigelow ML, Kahl J, Singh R, Coenen-Schimke J, Raghavakaimal S and Nair KS. Decline in skeletal muscle mitochondrial function with aging in humans. Proc Natl Acad Sci U S A 102: 5618-5623, 2005.

217. Sies H. Oxidative stress: oxidants and antioxidants. Exp Physiol 82: 291-295, 1997.

218. Siu PM, Pistilli EE and Alway SE. Apoptotic responses to hindlimb suspension in gastrocnemius muscles from young adult and aged rats. Am J Physiol Regul Integr Comp Physiol 289: R1015R1026, 2005.

219. Siu PM, Pistilli EE, Butler DC and Alway SE. Aging influences cellular and molecular responses of apoptosis to skeletal muscle unloading. Am J Physiol Cell Physiol 288: C338-C349, 2005.

220. Siu PM, Pistilli EE, Murlasits Z and Alway SE. Hindlimb unloading increases muscle content of cytosolic but not nuclear Id2 and p53 proteins in young adult and aged rats. J Appl Physiol 100: 907-916, 2006. 
221. Smerdu V, Karsch-Mizrachi I, Campione M, Leinwand L and Schiaffino S. Type Ilx myosin heavy chain transcripts are expressed in type Ilb fibers of human skeletal muscle. Am J Physiol 267: C1723-C1728, 1994.

222. Snow MH. Myogenic cell formation in regenerating rat skeletal muscle injured by mincing. II. An autoradiographic study. Anat Rec 188: 201-217, 1977.

223. St Pierre J, Buckingham JA, Roebuck SJ and Brand MD. Topology of superoxide production from different sites in the mitochondrial electron transport chain. J Biol Chem 277: 44784-44790, 2002.

224. Stevens L, Gohlsch B, Mounier Y and Pette D. Changes in myosin heavy chain mRNA and protein isoforms in single fibers of unloaded rat soleus muscle. FEBS Lett 463: 15-18, 1999.

225. Stienen GJ, Kiers JL, Bottinelli R and Reggiani C. Myofibrillar ATPase activity in skinned human skeletal muscle fibres: fibre type and temperature dependence. J Physiol 493 ( Pt 2): 299-307, 1996.

226. Su HC, Hung LM and Chen JK. Resveratrol, a red wine antioxidant, possesses an insulin-like effect in streptozotocin-induced diabetic rats. Am J Physiol Endocrinol Metab 290: E1339-E1346, 2006.

227. Suetta C, Hvid LG, Justesen L, Christensen U, Neergaard K, Simonsen L, Ortenblad N, Magnusson SP, Kjaer M and Aagaard P. Effects of aging on human skeletal muscle after immobilization and retraining. J Appl Physiol 107: 1172-1180, 2009. 
228. Tajbakhsh S, Rocancourt D, Cossu G and Buckingham M. Redefining the genetic hierarchies controlling skeletal myogenesis: Pax-3 and Myf-5 act upstream of MyoD. Cell 89: 127-138, 1997.

229. Takahashi M, Kubo T, Mizoguchi A, Carlson CG, Endo K and Ohnishi K. Spontaneous muscle action potentials fail to develop without fetal-type acetylcholine receptors. EMBO Rep 3: 674681, 2002.

230. Talmadge RJ, Roy RR and Edgerton VR. Distribution of myosin heavy chain isoforms in nonweight-bearing rat soleus muscle fibers. J Appl Physiol 81: 2540-2546, 1996.

231. Tatsumi R, Anderson JE, Nevoret CJ, Halevy $\mathbf{O}$ and Allen RE. HGF/SF is present in normal adult skeletal muscle and is capable of activating satellite cells. Dev Biol 194: 114-128, 1998.

232. Tatsumi R, Liu X, Pulido A, Morales M, Sakata T, Dial S, Hattori A, Ikeuchi Y and Allen RE. Satellite cell activation in stretched skeletal muscle and the role of nitric oxide and hepatocyte growth factor. Am J Physiol Cell Physiol 290: C1487-C1494, 2006.

233. Templeton GH, Sweeney HL, Timson BF, Padalino M and Dudenhoeffer GA. Changes in fiber composition of soleus muscle during rat hindlimb suspension. J Appl Physiol 65: 1191-1195, 1988.

234. Ten Broek RW, Grefte S and Von den Hoff JW. Regulatory factors and cell populations involved in skeletal muscle regeneration. J Cell Physiol 224: 7-16, 2010. 
235. Thomason DB and Booth FW. Atrophy of the soleus muscle by hindlimb unweighting. J App/ Physiol 68: 1-12, 1990.

236. Tissenbaum HA and Guarente L. Increased dosage of a sir-2 gene extends lifespan in Caenorhabditis elegans. Nature 410: 227-230, 2001.

237. Tonkin J, Villarroya F, Puri PL and Vinciguerra M. SIRT1 signaling as potential modulator of skeletal muscle diseases. Curr Opin Pharmacol 2012.

238. Trappe S, Creer A, Minchev K, Slivka D, Louis E, Luden N and Trappe T. Human soleus single muscle fiber function with exercise or nutrition countermeasures during 60 days of bed rest. Am J Physiol Regul Integr Comp Physiol 294: R939-R947, 2008.

239. Treisman J, Harris E and Desplan C. The paired box encodes a second DNA-binding domain in the paired homeo domain protein. Genes Dev 5: 594-604, 1991.

240. van Loo G, Saelens X, van Gurp M, MacFarlane M, Martin SJ and Vandenabeele P. The role of mitochondrial factors in apoptosis: a Russian roulette with more than one bullet. Cell Death Differ 9: 1031-1042, 2002.

241. Verdijk LB, Snijders T, Beelen M, Savelberg HH, Meijer K, Kuipers H and van Loon LJ. Characteristics of muscle fiber type are predictive of skeletal muscle mass and strength in elderly men. J Am Geriatr Soc 58: 2069-2075, 2010. 
242. Vinciguerra $\mathbf{M}$, Fulco $\mathbf{M}$, Ladurner $\mathbf{A}$, Sartorelli $\mathbf{V}$ and Rosenthal $\mathbf{N}$. SirT1 in muscle physiology and disease: lessons from mouse models. Dis Model Mech 3: 298-303, 2010.

243. Volonte D, Liu Y and Galbiati F. The modulation of caveolin-1 expression controls satellite cell activation during muscle repair. FASEB J 19: 237-239, 2005.

244. Wallace DC. Mitochondrial DNA mutations in disease and aging. Environ Mol Mutagen 51: 440450, 2010.

245. Walther C, Guenet JL, Simon D, Deutsch U, Jostes B, Goulding MD, Plachov D, Balling R and Gruss P. Pax: a murine multigene family of paired box-containing genes. Genomics 11: 424-434, 1991.

246. Waters DL, Baumgartner RN, Garry PJ and Vellas B. Advantages of dietary, exercise-related, and therapeutic interventions to prevent and treat sarcopenia in adult patients: an update. Clin Interv Aging 5: 259-270, 2010.

247. Weiss A, Schiaffino S and Leinwand LA. Comparative sequence analysis of the complete human sarcomeric myosin heavy chain family: implications for functional diversity. J Mol Biol 290: 6175, 1999.

248. Welle S, Bhatt $\mathrm{K}$ and Thornton CA. High-abundance mRNAs in human muscle: comparison between young and old. J Appl Physiol 89: 297-304, 2000. 
249. Wenz T, Rossi SG, Rotundo RL, Spiegelman BM and Moraes CT. Increased muscle PGC-1alpha expression protects from sarcopenia and metabolic disease during aging. Proc Natl Acad Sci U S A 106: 20405-20410, 2009.

250. Widrick JJ, Trappe SW, Blaser CA, Costill DL and Fitts RH. Isometric force and maximal shortening velocity of single muscle fibers from elite master runners. Am J Physiol 271: C666C675, 1996.

251. Widrick JJ, Trappe SW, Costill DL and Fitts RH. Force-velocity and force-power properties of single muscle fibers from elite master runners and sedentary men. Am J Physiol 271: C676-C683, 1996.

252. Wu X, Baer LA, Wolf SE, Wade CE and Walters TJ. The impact of muscle disuse on muscle atrophy in severely burned rats. J Surg Res 164: e243-e251, 2010.

253. Wu Z, Puigserver P, Andersson U, Zhang C, Adelmant G, Mootha V, Troy A, Cinti S, Lowell B, Scarpulla RC and Spiegelman BM. Mechanisms controlling mitochondrial biogenesis and respiration through the thermogenic coactivator PGC-1. Cell 98: 115-124, 1999.

254. Wyke SM, Russell ST and Tisdale MJ. Induction of proteasome expression in skeletal muscle is attenuated by inhibitors of NF-kappaB activation. Br J Cancer 91: 1742-1750, 2004.

255. Wyke SM and Tisdale MJ. Induction of protein degradation in skeletal muscle by a phorbol ester involves upregulation of the ubiquitin-proteasome proteolytic pathway. Life Sci 78: 2898-2910, 2006. 
256. Xu J, Hwang JC, Lees HA, Wohlgemuth SE, Knutson MD, Judge AR, Dupont-Versteegden EE, Marzetti E and Leeuwenburgh C. Long-term perturbation of muscle iron homeostasis following hindlimb suspension in old rats is associated with high levels of oxidative stress and impaired recovery from atrophy. Exp Gerontol 47: 100-108, 2012.

257. Yasuda N, Glover El, Phillips SM, Isfort RJ and Tarnopolsky MA. Sex-based differences in skeletal muscle function and morphology with short-term limb immobilization. J Appl Physiol 99: 1085-1092, 2005.

258. Yasuhara K, Ohno Y, Kojima A, Uehara K, Beppu M, Sugiura T, Fujimoto M, Nakai A, Ohira Y, Yoshioka T and Goto K. Absence of heat shock transcription factor 1 retards the regrowth of atrophied soleus muscle in mice. J Appl Physiol 2011.

259. Zaccagnini G, Martelli F, Magenta A, Cencioni C, Fasanaro P, Nicoletti C, Biglioli P, Pelicci PG and Capogrossi MC. p66(ShcA) and oxidative stress modulate myogenic differentiation and skeletal muscle regeneration after hind limb ischemia. J Biol Chem 282: 31453-31459, 2007.

260. Zammit PS, Golding JP, Nagata Y, Hudon V, Partridge TA and Beauchamp JR. Muscle satellite cells adopt divergent fates: a mechanism for self-renewal? J Cell Biol 166: 347-357, 2004.

261. Zhang J, Chen L, Zheng J, Zeng T, Li H, Xiao H, Deng X and Hu X. The protective effect of resveratrol on islet insulin secretion and morphology in mice on a high-fat diet. Diabetes Res Clin Pract 2012. 